


\title{
Coupling of GTP hydrolysis by EF-G to tRNA and mRNA translocation through the ribosome
}

\author{
Dissertation \\ for the degree "Doctor rerum naturalium" \\ in the Molecular Biology Program \\ at the Georg-August Universität Göttingen, \\ Faculty of Mathematics and Natural Sciences
}

submitted by

Carlos Eduardo Lima da Cunha

from São Paulo, Brazil

Göttingen, July 2013 


\section{Thesis Committee:}

Prof. Dr. Marina V. Rodnina

Department of Physical Biochemistry

Max-Planck Institute for Biophysical Chemistry

Prof. Dr. Holger Stark

3D Cryo-Electron Microscopy

Max-Planck Institute for Biophysical Chemistry

Prof. Dr. Kai Tittmann

Department of Bioanalytics

Georg-August Universität Göttingen

Date of the defense: June, $19^{\text {th }} 2013$ 


\section{Affidavit}

I hereby declare that I prepared the dissertation entitled "Coupling of GTP hydrolysis by EF-G to tRNA and mRNA translocation through the ribosome" on my own and with no other sources and aids than quoted.

Carlos Eduardo Lima da Cunha

Göttingen, July, $4^{\text {th }} 2013$ 


\section{Acknowledgments}

All these years in Göttingen have been amazing, and I certainly did not only grow scientifically, but also as a person. Of course this does not come by itself with the passage of time, but through experiences shared with those around me.

First, I would like to thank Frank Peske, Wolfgang Wintermeyer and Marina Rodnina for introducing me into the ribosome world - it is a very fascinating field, which I had never thought I would end up working in. I would like to specially thank Frank for taking all the time needed to talk with me and also for letting me take my time when needed. I would also like to thank Anja Lehwess-Litzmann and Sarah Adio for the nice scientific chats. Furthermore, I would like to thank the members of my thesis committee Holger Stark and Kai Tittmann, for taking some of their time to listen to and discuss my research.

The day to day in the lab is made more vibrant when one works around cool people, and I am fortunate enough to have many of them around me. I would like to thank Irena, Aki, Michael, Mariam, Ev and Heena for such a great time. Also, I would like to thank Neva for joining me in the "Cool Corner" and the "Victory Chocolate Committee".

Living in Göttingen is more than the daily lab life. I would like to thank Koray, Sinem, Chepe, Melanie and Iris for making life outside the lab as fun and eventful as it can be. Getting to know them all already in our first year in Göttingen was amazing and our traditional Easter trips are memorable.

I would like to specially thank the $P \& C$ team of the Horizons meeting - what started out as an accidentally assembled working team turned out to be a great circle of friends. I would like to thank Jenny for all the cool moments during football matches and parties and for agreeing that Gómez should not be allowed close to any ball. Also, I would like to thank David for being a good friend since even before we met in Göttingen, for the very relaxing table tennis sessions in the late afternoon and for testing his delicious cooking experiments with me. At last, I would like to thank Simone for being great company whose laugh always cheers me up, for all great chatty and dancing nights out and for being there for me.

Finally, I would like to thank my family (Sueli, Roberto, Arthur and Débora) for all their 
support throughout these years. I would like to thank my parents for always encouraging me and stimulating my curiosity. They never ceased to try to answer all the questions I was asking - and there were not few! Of course, I also want to thank my brother and my sister for all the great times we have together and for making me feel closer to home with our (often hilarious) chats, despite the distance. 


\section{Contents}

1 Abstract 12

2 Introduction 13

2.1 General overview . . . . . . . . . . . . . . . . . . . . . . 13

2.2 The prokaryotic ribosome $\ldots \ldots \ldots \ldots \ldots \ldots$

2.3 Elongation factors $\ldots \ldots \ldots \ldots \ldots \ldots$

2.3.1 Elongation factor $\mathrm{G} \ldots \ldots \ldots \ldots \ldots$

2.3.2 EF-G as a GTPase molecular motor . . . . . . . . . . . . 22

2.3.3 Role of GTP hydrolysis . . . . . . . . . . . . . . . . . . . 24

2.4 EF-G and ribosome dynamics during translocation $\ldots \ldots \ldots . . \ldots 26$

2.4.1 Classical and hybrid states . . . . . . . . . . . . . . . . 28

2.4.2 Synchronous translocation requires GTP hydrolysis . . . . . . . 29

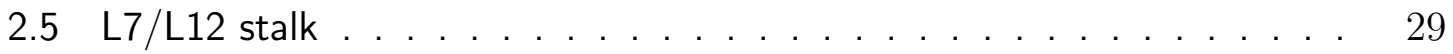

2.5 .1 Factor recruitment . . . . . . . . . . . . . . . . . . 31

2.5 .2 GTPase activation . . . . . . . . . . . . . . 31

2.5.3 The stalk and translocation . . . . . . . . . . . . . . . 32

3 Material and Methods 33

3.1 Buffers and reagents . . . . . . . . . . . . . . . . 33

3.2 Ribosomes, mRNAs, tRNAs, and translation factors . . . . . . . 33

3.3 BODIPY-Met-tRNA ${ }^{f M e t} \ldots \ldots \ldots \ldots \ldots \ldots \ldots$

3.4 Expression and purification of EF-G and EF-G mutants . . . . . . 34

3.4.1 Wild type and $\mathrm{H} 91 \mathrm{~A}$ mutation . . . . . . . . . . . . . 34 
3.4.2 H583K and $\Delta 4 / 5$ mutation . . . . . . . . . . . . . . . . . 35

3.5 Growth curves . . . . . . . . . . . . . . . . . . . . . 35

3.6 Labeling of $30 \mathrm{~S}$ subunits . . . . . . . . . . . . . . . . . . . 36

3.7 Ribosome complexes . . . . . . . . . . . . . . . . . . . . 36

3.8 Turnover GTP hydrolysis . . . . . . . . . . . . . . . . . . . . . . . . 37

3.9 Puromycin reaction . . . . . . . . . . . . . . . . . . 38

3.10 Rapid kinetics methods . . . . . . . . . . . . . . . . . . . . . . . . . . 38

3.10.1 Single-round GTP hydrolysis . . . . . . . . . . . . . . . . . . . 38

3.10 .2 Pi release . . . . . . . . . . . . . . . . . . . . . . . . 38

3.10.3 Single-round translocation . . . . . . . . . . . . . . . . . . . 39

3.10.4 EF-G ribosome complex formation and dissociation . . . . . . . . 39

3.11 EF-G-ribosome pull-down assay . . . . . . . . . . . . . . . . . . . . . . 39

3.12 Tripeptide formation . . . . . . . . . . . . . . . . . . . . . 40

3.13 Nucleotide binding . . . . . . . . . . . . . . . . . . . . . . . . . . . . 40

4 Results $\quad 41$

4.1 Construction of the GTPase-deficient EF-G mutant . . . . . . . . . . . . 41

4.2 Translocation is slow without GTP hydrolysis . . . . . . . . . . . . . . . 44

4.3 Direct observation of $30 \mathrm{~S}$ and $50 \mathrm{~S}$ translocation . . . . . . . . . . . 49

4.4 Role of GTP hydrolysis . . . . . . . . . . . . . . . . . . . . . . . 51

4.5 The complex of ribosomes with EF-G(H91A)-GTP is very stable . . . . . 54

5 Discussion $\quad 58$

58

5.2 GTP hydrolysis and a functional domain 4 are required for synchronous translocation ...................... 63

5.3 Conclusion . . . . . . . . . . . . . . . . . . . . . 67

6 References $\quad 69$

$\begin{array}{llr}7 & \text { Biography } & 83\end{array}$ 


\section{List of Figures}

2-1 Elongation cycle . . . . . . . . . . . . . . . . . . . . . 14

2-2 The prokaryotic ribosome $\ldots \ldots \ldots \ldots \ldots$

2-3 Ribosome dynamics . . . . . . . . . . . . . . . . . . . 17

2-4 Conserved domains in translational GTPases . . . . . . . . . . . . . 19

2-5 Contacts between EF-G and the ribosome in the post-translocation state 20

2-6 EF-G dynamics . . . . . . . . . . . . . . . . . . . . . . . . . . . 21

2-7 L7/L12 stalk . . . . . . . . . . . . . . . . . . . . 30

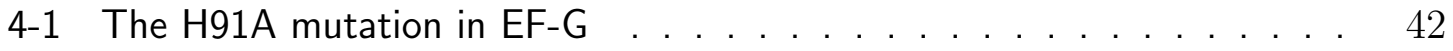

4-2 Multiple turnover GTP hydrolysis . . . . . . . . . . . . 43

$4-3$ Pi release . . . . . . . . . . . . . . . . . . . . . 43

4-4 Single-round GTP hydrolysis . . . . . . . . . . . . . . . . 43

$4-5$ Nucleotide binding . . . . . . . . . . . . . . . . . 44

$4-6$ Puromycin assay . . . . . . . . . . . . . . . . . 45

4-7 Fluorescence labels . . . . . . . . . . . . . . . . . . . . 45

4-8 Proflavin fluorescence . . . . . . . . . . . . . . . . 46

$4-9$ Fluorescein fluorescence . . . . . . . . . . . . . . . . 46

4-10 Alexa 488 fluorescence . . . . . . . . . . . . . . . . . . . 46

$4-11$ Kinetics of $50 \mathrm{~S}$ and $30 \mathrm{~S}$ translocation . . . . . . . . . 50

4-12 Inhibition of translocation by mutations in EF-G . . . . . . . 52

4-13 Concentration dependence of apparent rate constants of translocation promoted by EF-G(H91A) . . . . . . . . . . . . . . . 53

$4-14$ EF-G stability on the ribosome $\ldots \ldots \ldots \ldots \ldots \ldots$ 
4-15 EF-G binding to pre-translocation complex . . . . . . . . . . . . . 54

4-16 EF-G binding to the ribosome . . . . . . . . . . . . . 55

4-17 EF-G and EF-Tu competing binding site . . . . . . . . . . . . . 55

5-1 Schematic of translocation . . . . . . . . . . . . . . . . . . 65

5-2 Translocation Model . . . . . . . . . . . . . . . . . . . . 66 


\section{List of Tables}

4.1 Rate constants of elemental steps of translocation . . . . . . . . . . . 47

4.2 Rate constants of $50 \mathrm{~S}$ and $30 \mathrm{~S}$ translocation . . . . . . . . . . 51 


\section{1}

\section{Abstract}

Translocation is the concerted movement of the messenger RNA (mRNA) and the transfer RNA (tRNA) through the ribosome. Although spontaneous in vitro, this process is catalyzed by the translational GTPase elongation factor G (EF-G). How EF-G couples the energy released by GTP hydrolysis to translocation is yet unknown. Furthermore, a mechanistic understanding of translocation is also missing, e.g. the timing of tRNA translocation of the two ribosomal subunits is not known. To address these questions, we designed an EF-G mutant that is unable to hydrolyze GTP, EF-G(H91A), while binding to nucleotides and the ribosome with similar affinities as the wild-type. We find that the rate of translocation is reduced in the presence of EF-G(H91A) as compared to the wildtype EF-G, but is higher than spontaneous translocation. H91A also remains strongly bound to the ribosome and does not dissociate even under non-equilibrium conditions. This suggests that EF-G works in a dual energy regime on the ribosome: (i) as a probabilistic molecular motor biasing the forward tRNA movement through conformational constraints, (ii) as switch-type GTPase dissociating from the ribosome once the GDPbound conformational state is reached. Additionally, using EF-G(H91A), we demonstrate that translocation occurs in a synchronous way on the two ribosomal subunits and that EF-G binding only causes partial 50 S subunit translocation. The energy of GTP hydrolysis is coupled to translocation of the $30 \mathrm{~S}$ subunit and the completion of translocation on the $50 \mathrm{~S}$ subunit. Together these results show that EF-G plays a dual role during translocation and that GTP hydrolysis has a key role in synchronizing this process. 


\section{2}

\section{Introduction}

\subsection{General overview}

The ribosome is a macromolecular machine that synthesizes proteins in the cell by translating genetic information encoded in the messenger RNA (mRNA) into a sequence of amino acids. Different translation factors interact transiently with the ribosome and assist it on each step of translation.

For protein synthesis to begin, ribosome subunits, the initiator transfer RNA (tRNA) and the mRNA assisted by initiation factors assemble, forming a ribosomal initiation complex, containing an fMet-tRNA ${ }^{f M e t}$ in the $\mathrm{P}$ site and a vacant $\mathrm{A}$ site, which is ready for the elongation cycle (Milon and Rodnina, 2012). The first step of elongation is the accommodation of the correct coding aminoacylated-tRNA (aa-tRNA) in the A site (Figure 1-1). The decoding is assisted by the translational GTPase elongation factor Tu (EF-Tu) in complex with an aa-tRNA and GTP, which constitute a ternary complex. Upon codon-anticodon recognition, GTP is hydrolyzed, and EF-Tu dissociates from the ribosome. The accommodation of the aa-tRNA in the A site leads its acceptor arm to bind into the peptidyl transferase center (PTC), followed by a rapid peptide bond formation, resulting in a deacylated tRNA at the $\mathrm{P}$ site and a peptidyl-tRNA extended by one amino acid in the $A$ site. The last step within the elongation cycle is reached when the mRNA/tRNA complex is translocated by the translational GTPase elongation factor $G(E F-G)$. During translocation, the peptidyl-tRNA is moved from the $A$ site to the $P$ 
site; concomitantly, the mRNA is also moved by one codon. The $\mathrm{P}$ site is now occupied by a new peptidyl-tRNA, the $A$ site is vacant for the next elongation round. These three substeps of elongation are repeated until the entire coding sequence of the mRNA is translated, and a termination codon is reached. The translation process is terminated by the release of the peptide from the ribosome with the help of release factors 1,2 , and 3 and the dissociation of the ribosomal subunits promoted by the ribosome recycling factor and EF-G (Wintermeyer et al., 2004, Frank et al., 2007, Agirrezabala and Frank, 2009, Rodnina and Wintermeyer, 2011).
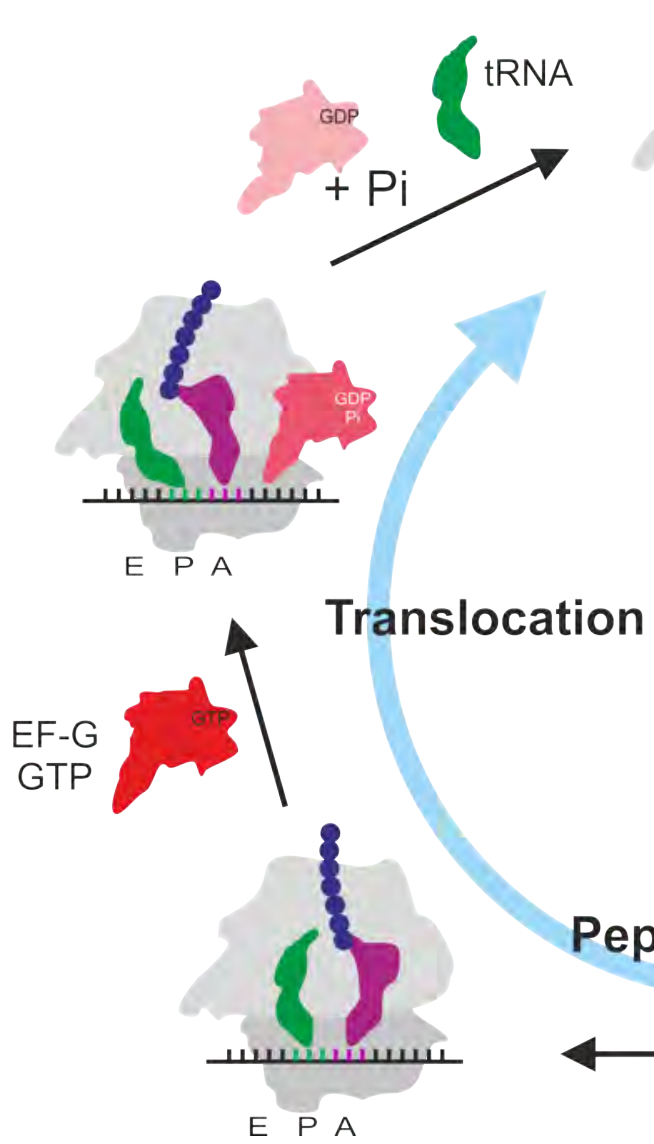

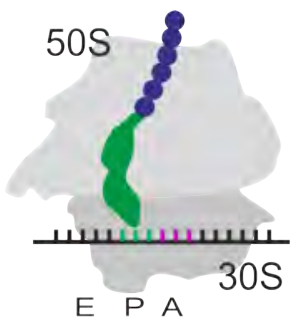

\section{Peptidyl transfer}
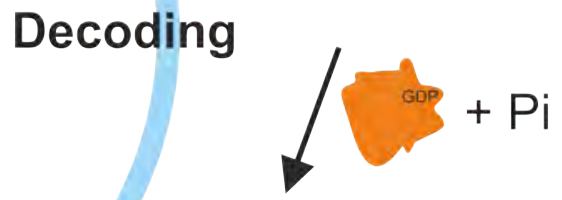

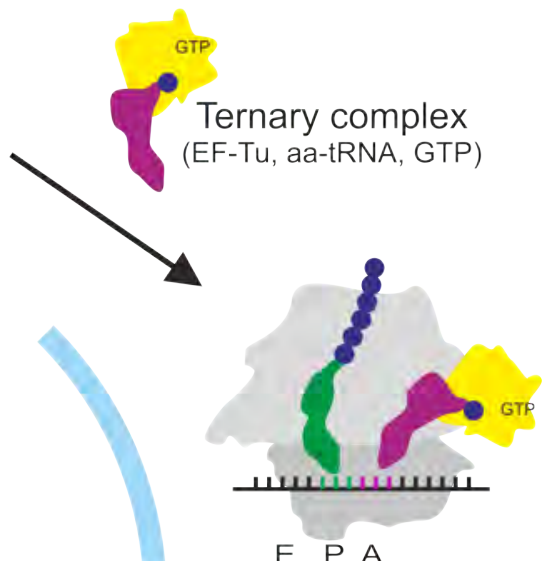

Decoding
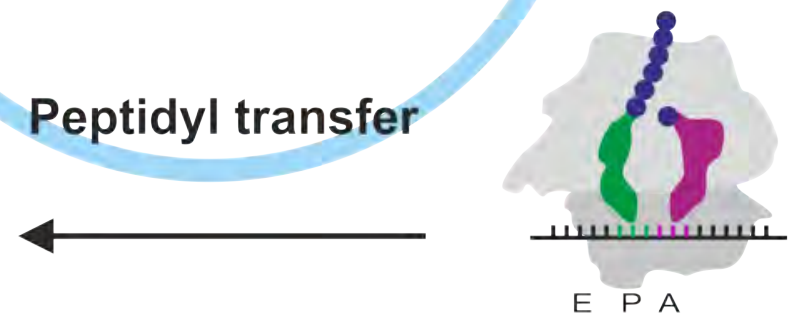

Figure 2-1: Elongation cycle. Elongation is a repetitive cycle in which an aa-tRNA is selected according to the codon in the mRNA with the help of EF-Tu (decoding), the peptide bond is formed to the preceding amino acid (peptidyl transfer), and then the mRNA/tRNA complex is translocated with the help of EF-G (translocation).

The ribosome consists of two distinct subunits, the $30 \mathrm{~S}$ and the $50 \mathrm{~S}$ subunits in prokaryotes. On the $30 \mathrm{~S}$ subunit the decoding of the mRNA takes place by monitoring 
the codon-anticodon interactions; the 50S subunit provides the chemical environment for peptide bond formation in the peptidyl transferase center (PTC). When the subunits are joined forming the $70 \mathrm{~S}$ ribosome, three binding sites for the tRNA are formed: the A site (binding site of aa-tRNA), the $P$ site (binding site of peptidyl-tRNA) and the $E$ site (exit site of the deacylated tRNA). Each of these sites is functionally different (Schmeing and Ramakrishnan, 2009).

The process of translation is dynamic, and it requires the ribosome and many of its ligands to undergo many structural changes, which when hindered block mRNA/tRNA complex translocation and thus translation. Conformational changes, such as intersubunit rotation by the ribosome (Horan and Noller, 2007) and a hinge-like movement of EF-G (Stark et al., 2000, Peske et al., 2000), are essential for translocation and will be discussed in detail in the next sections.

\subsection{The prokaryotic ribosome}

The prokaryotic ribosome is a large macromolecular machine composed of 3 RNA molecules and more than 50 proteins. It has a diameter of about $20 \mathrm{~nm}$ and a molecular weight of 2.5 MDa. The $50 \mathrm{~S}$ subunit is composed of the $5 \mathrm{~S}$ ribosomal RNA (rRNA), the 23S rRNA, and 31 proteins (denominated L proteins), while the $30 S$ subunit is significantly smaller with only one RNA molecule (16S rRNA) and 21 proteins (called S proteins) (Figure 1-2) (Shoji et al., 2009).

The ribosome is a ribozyme, because its catalytic properties are mediated by solely RNA groups. Proteins seem to have a structural role providing a scaffold and neutralizing charges of the large RNA molecules (Rodnina et al., 2007). On the $30 \mathrm{~S}$ subunit, the $16 \mathrm{~S}$ rRNA contains the anti-Shine-Dalgarno sequence which stabilizes the mRNA association with the ribosome during initiation (the Shine-Dalgarno sequence is a short sequence located about 8 bases upstream of the start codon that helps to recruit the ribosome to the mRNA). The $16 \mathrm{~S}$ rRNA is also involved in the stabilization of the codon-anticodon interactions in the decoding center - broadly speaking, when this interaction is not fully achieved the tRNA is rejected (Rodnina and Wintermeyer, 2001, Schmeing and Ramakr- 


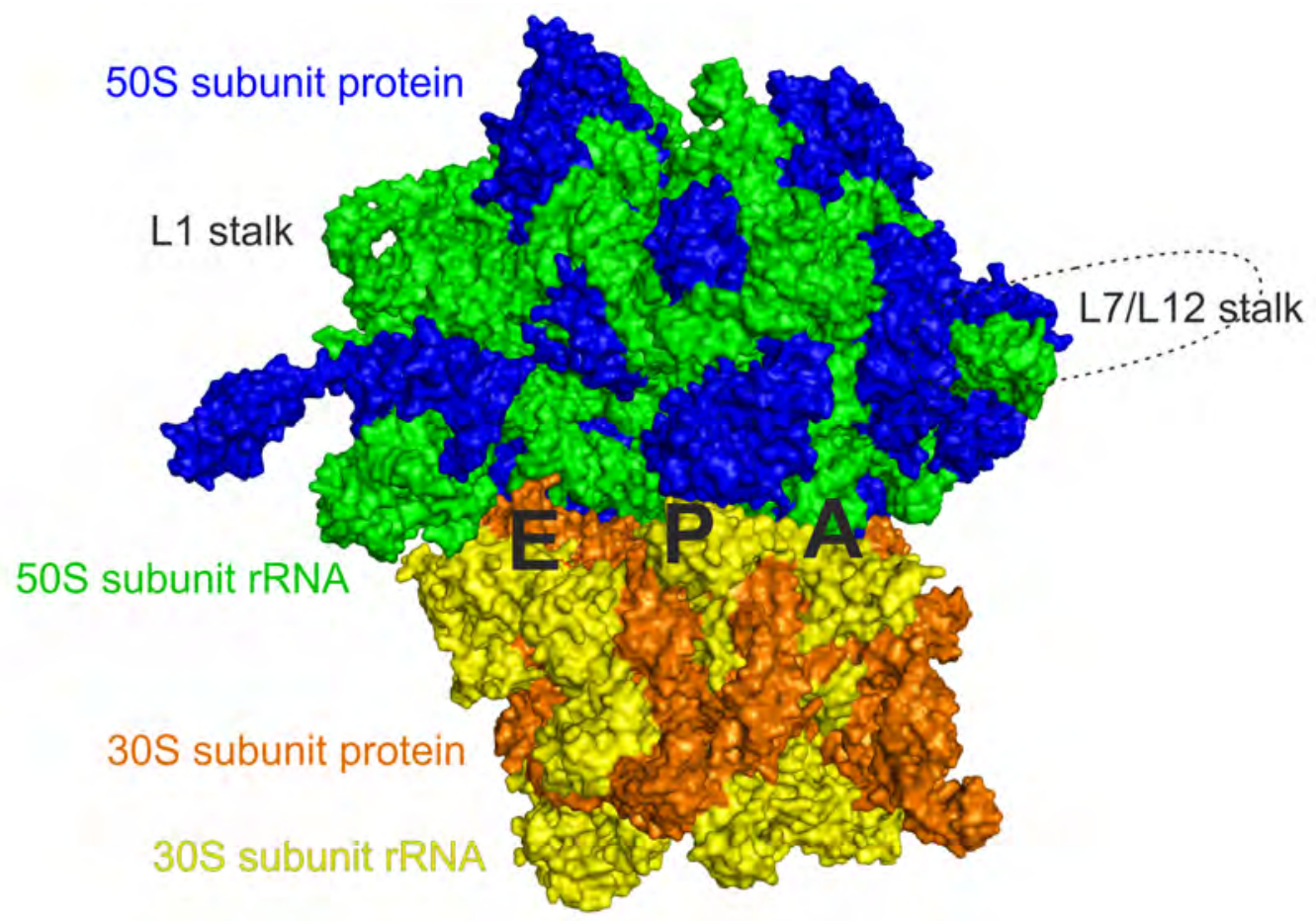

Figure 2-2: The prokaryotic ribosome. The prokaryotic ribosome is composed of two subunits: the large 50S subunit and the small 30S subunit. The $50 \mathrm{~S}$ subunit is composed of $5 \mathrm{~S}$ rRNA, 23S rRNA (both in green), and 31 proteins (blue). The $30 \mathrm{~S}$ subunit consists of $16 \mathrm{~S}$ rRNA (orange) and 21 proteins (yellow). Image made from PDB files 2WDK and 2WDL.

ishnan, 2009). On the $50 \mathrm{~S}$ subunit, the $23 \mathrm{~S}$ rRNA has two distinct functions: It acts as a catalyst of peptide bond formation at the PTC as well as a GAP (GTP activating protein) for translational GTPases (Mohr et al., 2002). Much of the function of the $5 S$ rRNA remains unknown - so far the notion is that it interacts with many different proteins on the ribosome most likely stabilizing the entire ribosomal structure (Barciszewska et al., 2001).

Ribosomes are remarkably dynamic (Figure 1-3). One of the largest and most global movements is ribosome ratcheting: a counterclockwise rotation of the $30 \mathrm{~S}$ subunit with respect to the 50S subunit, which is essential for tRNA and mRNA translocation (Horan and Noller, 2007, Frank and Agrawal, 2000). Moreover, the ribosome encompasses two very flexible stalks: the $\mathrm{L} 1$ and the $\mathrm{L} 7 / \mathrm{L} 12$ stalks. The $\mathrm{L} 1$ stalk is thought to mediate tRNA translocation by actively removing the deacylated-tRNAs from the $E$ site (Trabuco et al., 2010). The L7/L12 stalk, located on the opposite side of the L1 stalk, close to the 


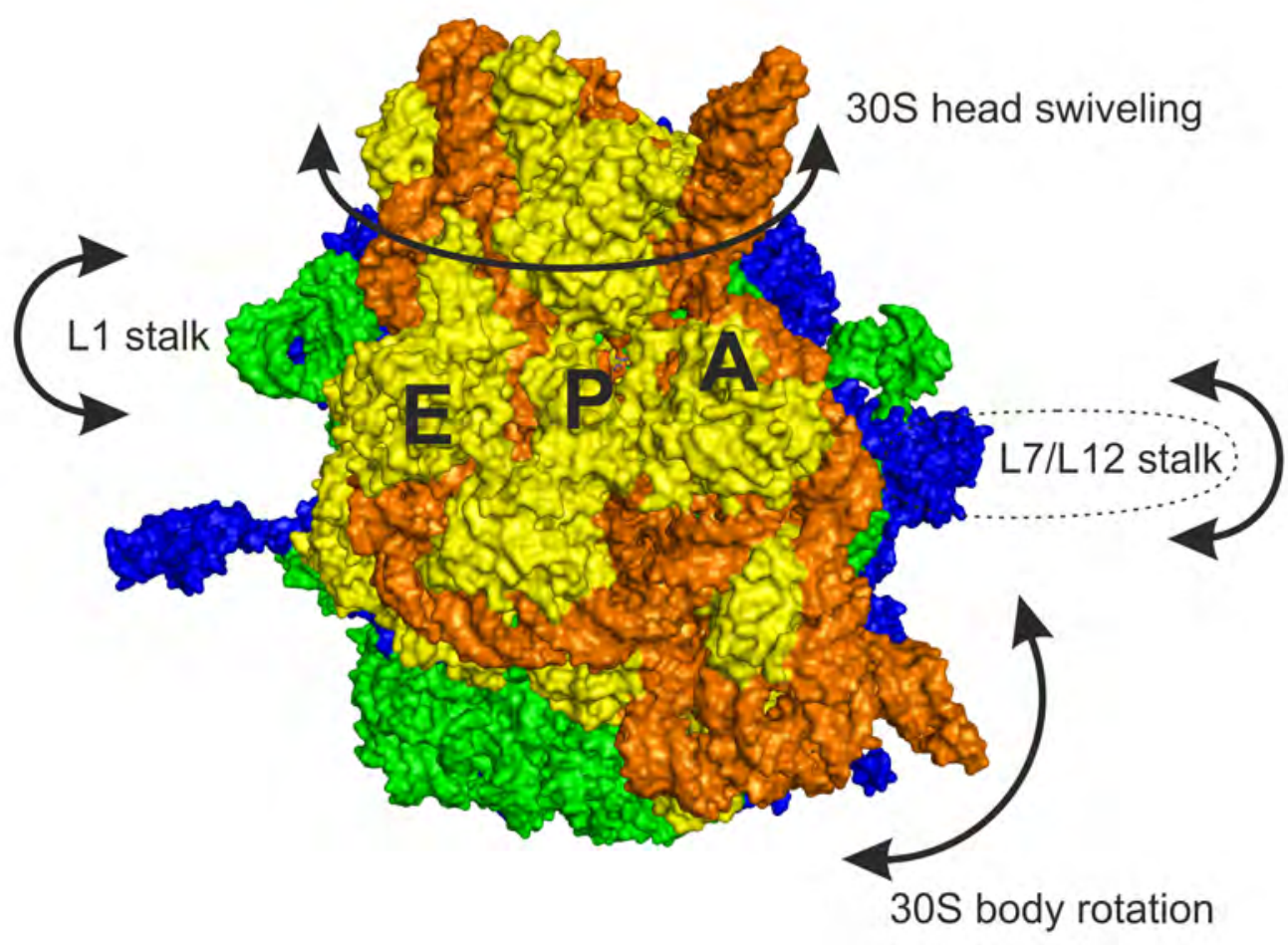

Figure 2-3: Ribosome dynamics. The ribosome is highly dynamic with two large scale movements: the $30 \mathrm{~S}$ subunit ratcheting, the $30 \mathrm{~S}$ subunit head swiveling. Two very flexible stalks are also present on the ribosome: the L1 and the L7/L12 stalks. The L7/L12 stalk is very flexible and normally it is not resolved in X-ray structures and only partial density for it is seen in cryo-EM reconstructions. Ribosome structure and color code as in Figure 1-2.

entrance to the A site, is a highly flexible structure, and its function is related to factor recruitment and GTPase activation - its role will be further discussed in section 1.5 (Wahl and Moller, 2002). Finally, the other large ribosomal movement is a movement of the head of the $30 \mathrm{~S}$ subunit - the head swiveling. This movement is only loosely coupled to ratcheting (Fischer et al., 2010) and is believed to play a key role in mRNA/tRNA translocation (discussed in more details in next section 1.4). In addition, it may be responsible for the intrinsic helicase activity of the ribosome when melting mRNA secondary structures (Borovinskaya et al., 2007, Takyar et al., 2005, Rodnina and Wintermeyer, 2011).

In summary, the ribosome is a dynamic machine involved in fast and accurate translation. Ratcheting allows a high degree of tRNA movement, the L1 stalk removes tRNA from the $E$ site, the $L 7 / L 12$ stalk recruits and activates translation factors at the $A$ site, and head swiveling works by assisting mRNA/tRNA translocation on the $30 \mathrm{~S}$ subunit. 


\subsection{Elongation factors}

Elongation is facilitated by EF-Tu, EF-G, and, most recently discovered, EF-P (elongation factor P). EF-Tu is a translational GTPase involved in the decoding step during elongation (see section 1.1) (Wintermeyer et al., 2004). EF-G is the translational GTPase responsible for the fast translocation step (discussed further in the next section) and for subunit dissociation during ribosome recycling. At this final stage of translation, EF-G catalyzes, in a concerted manner with the ribosome recycling factor, the rapid dissociation of the post-termination ribosomal complex into subunits (Hirashima and Kaji, 1973, Wintermeyer et al., 2004). Finally, EF-P, whose role has only recently been understood, accelerates peptide bond formation on proline stretches, allowing fast incorporation of these amino acids by the translating ribosome (Doerfel et al., 2013, Ude et al., 2013). EF-Tu and EFG have highly conserved GTP binding domains (called G domains) (Caldon and March, 2003, Verstraeten et al., 2011), which can be used for comparative studies. Structural comparisons of the same motifs in different conditions allow a better understanding of the mechanism of GTP activation and hydrolysis by these factors.

\subsubsection{Elongation factor $G$}

EF-G is the key player in translocation, accelerating the process by five orders of magnitude while consuming one GTP molecule per round of translocation. EF-G is a $78 \mathrm{kDa}$ GTPase comprised of 6 domains, domain 1 (also called G domain) containing an insertion (G' domain) and domains 2 to 5 (Figure 1-4) (Verstraeten et al., 2011, Bourne et al., 1991). While domains 1 and 2 are conserved between EF-G, EF-Tu and other translational GTPases, domains 3 to 5 are present only in EF-G and a few other GTPases: domain 3 is conserved in the release factor 3 (RF-3) (Zhou et al., 2012), and domains 3 and 5 are conserved in LepA, a GTPase presumably catalyzing reverse-translocation (Evans et al., 2008). Additionally, domains 3 to 5 of EF-G are structurally similar to the tRNA part of the ternary complex formed by EF-Tu, tRNA and GTP (Figure 1-4) (Nissen et al., 2000).

Some of the post-translocation interactions between EF-G and the ribosome are known from cryo-electron microscopy and reconstructions (Connell et al., 2007, Ratje et al., 

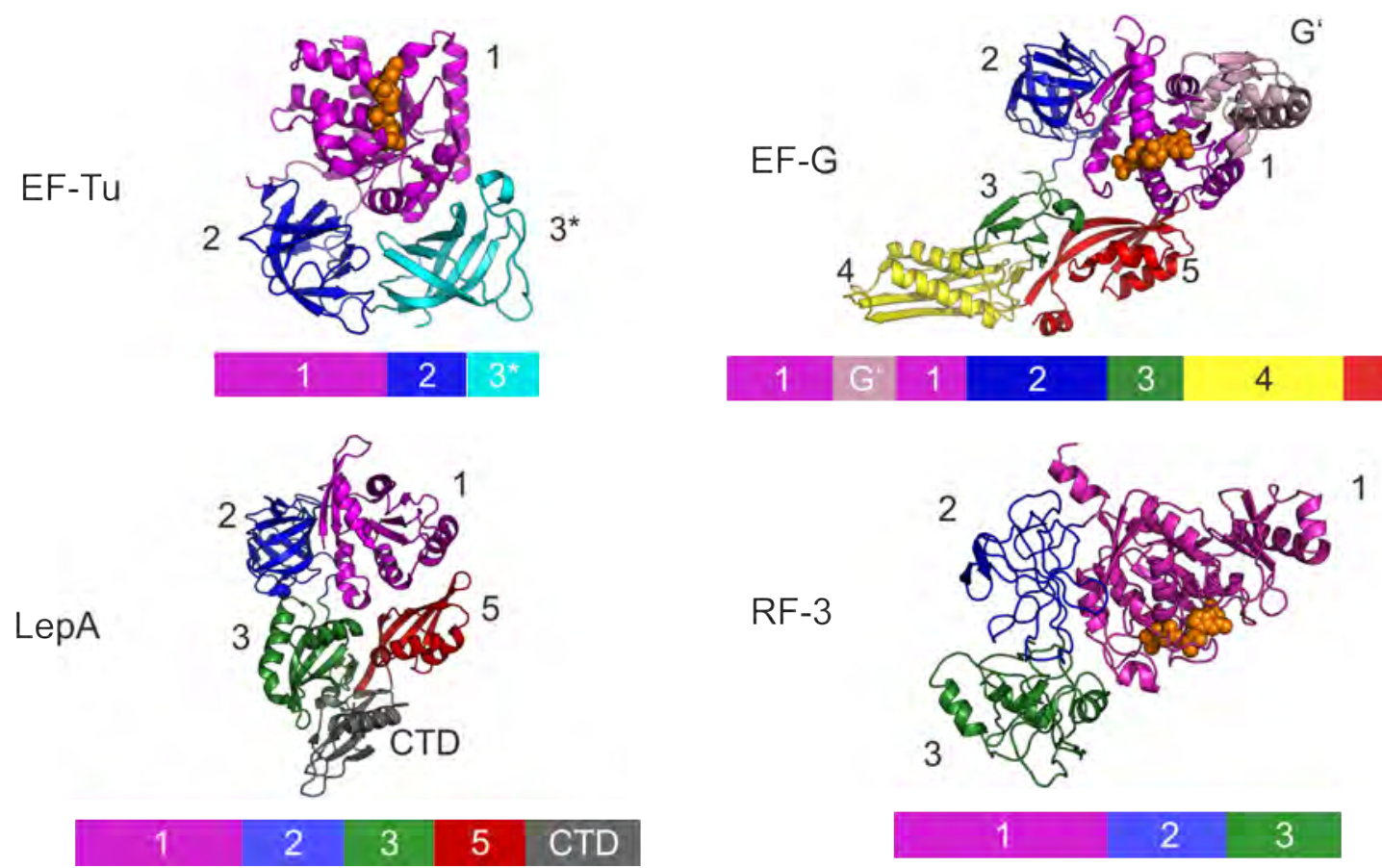

Figure 2-4: Conserved domains in translational GTPases. EF-Tu, EF-G, LepA and RF-3 are translational GTPases. Domains 1 and 2 are conserved in all these GTPases, while EF-Tu (PDB 1EXM) has a third specific domain $\left(3^{*}\right)$. Domain 4 is exclusive to EF-G (PDB 2BV3), performing a power stroke role during translocation. LepA (PDB 3CB4) shares domains 3 and 5 with EF-G, but has a unique C-terminal domain (CTD). RF-3 (PDB 3UOQ) consists only of three highly conserved domains 1 to 3 . Conserved domains are colored identically.

2010) and a crystal structure of EF-G blocked on the ribosome by addition of fusidic acid (Gao et al., 2009) (Figure 1-5). These structures provide hints on the mechanism of EF-G-dependent translocation; however, very little structural information is available so far regarding the early interactions of EF-G and the ribosome before translocation.

In post-translocation complexes, domains 1 and 5 of EF-G interact mainly with the $50 \mathrm{~S}$ subunit. Domain 1 contacts the GTPase-associated center (GAC) (composed of the sarcin-ricin loop (SRL) of $23 \mathrm{~S}$ rRNA and the ribosomal protein L7/L12), while domain 5 interacts with the 1060 region of $23 \mathrm{~S}$ rRNA (the binding region for the ribosomal protein L11). On the $30 \mathrm{~S}$ subunit, domain 2 contacts $16 \mathrm{~S}$ rRNA, domain 3 interact with S12 (one of the proteins in a pivotal position during intersubunit rotation) and domain 4 extends into the decoding center (Figures 1-5 and 1-6) (Stark et al., 2000, Frank and Agrawal, 2000). Comparative studies with the eukaryotic ortholog of EF-G, EF-2, show that domains 3 


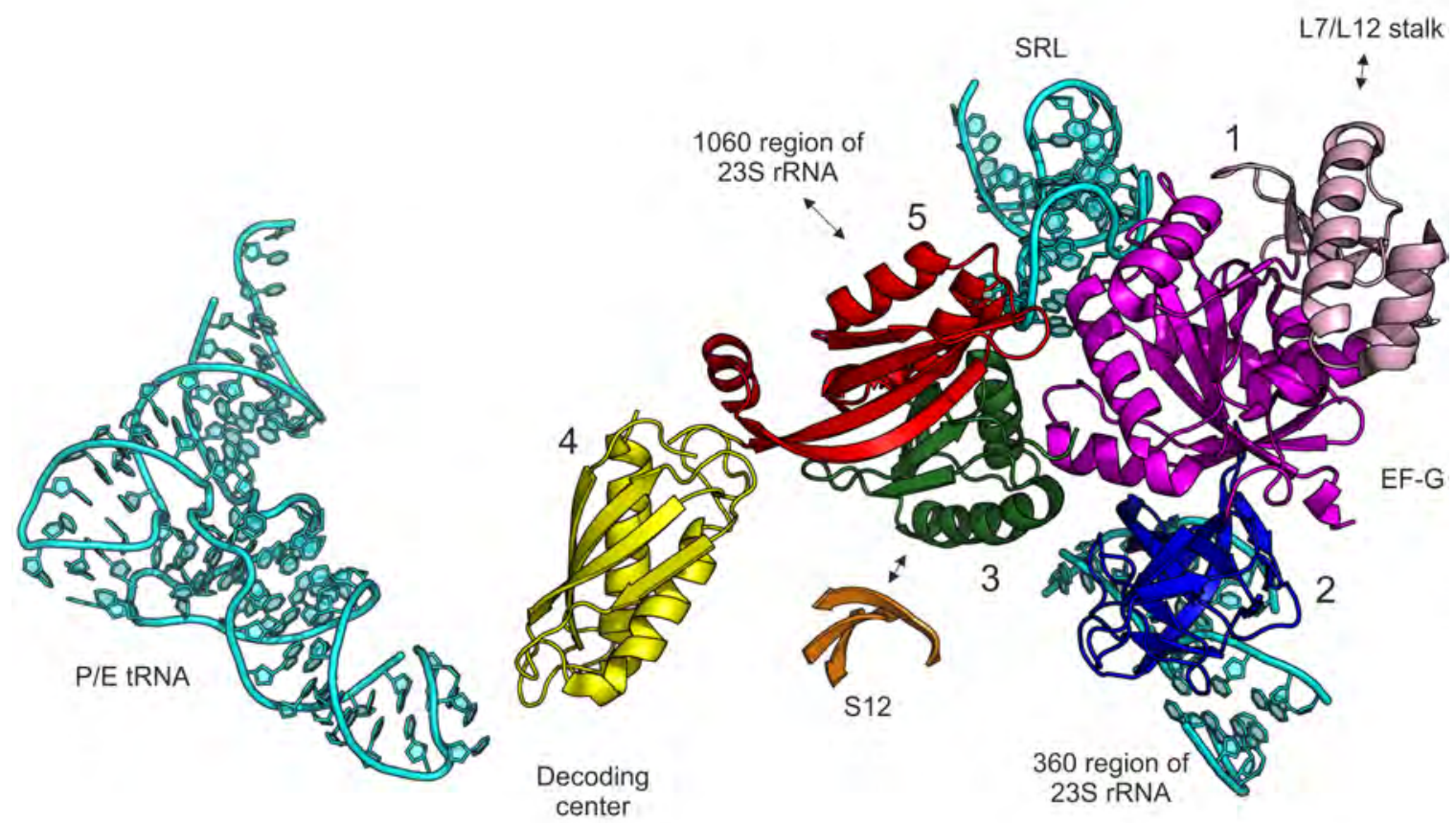

Figure 2-5: Contacts between EF-G and the ribosome in the posttranslocation state. Domains 1 and 5 of EF-G interact mainly with the $50 \mathrm{~S}$ subunit, in particular with domain 1 of EF-G contacting the sarcin-ricin loop (SRL) of 23S rRNA (cyan) and the C-terminal domain (CTD) of the ribosomal protein L7/L12 (light pink), while domain 5 interacts with other regions in 23S rRNA. On the $30 \mathrm{~S}$ subunit, domain 2 of EF-G contacts $16 \mathrm{~S}$ rRNA as well as $23 \mathrm{~S}$ rRNA, domain 3 interacts with S12 (orange) and domain 4 extends into the decoding center, where the tRNA (cyan) is located. Image based on PDB 2OM7. EF-G color coded according to EF-G domains as in Figure 1-4.

to 5 undergo a large hinge-like conformational change (around $25 \stackrel{\circ}{A}$ shift) when on the ribosome, after GTP hydrolysis in the complex stalled by an antibiotic sordarin (Spahn et al., 2004, Jorgensen et al., 2003). Such a structural change had also been previously observed in EF-G by Stark and colleagues in a cryo-EM study (Stark et al., 2000). Hence, these large conformational changes that EF-G, particularly domain 4, undergoes during translocation would impose a conformational constraint on the 305 subunit, which causes a structural change in $16 \mathrm{~S}$ rRNA, opening up room for domain 4 to contact the decoding center.

The destabilization of the interactions between 16S rRNA and the tRNA in the decoding center would loosen their interaction and thus ultimately lead to tRNA translocation 


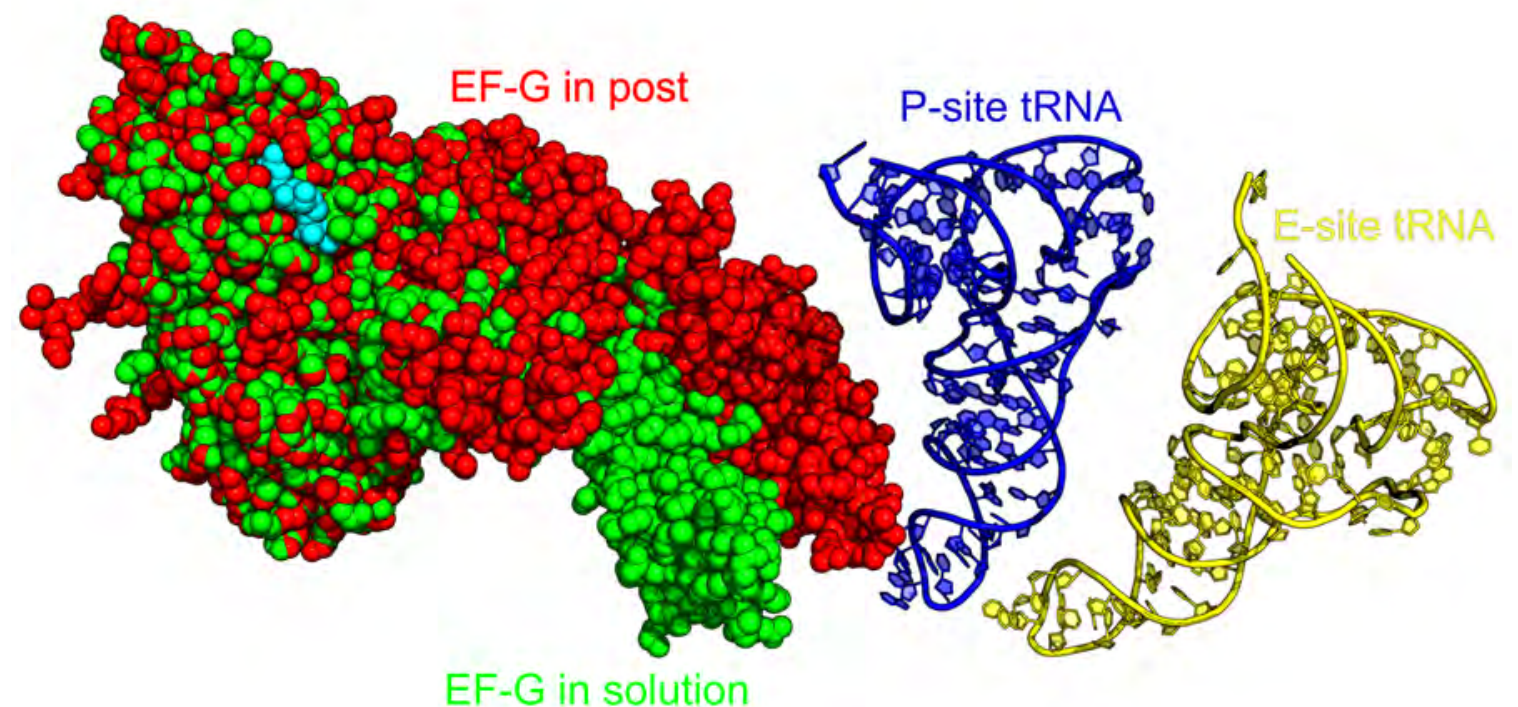

Figure 2-6: EF-G dynamics. EF-G in the post-translocation state stabilized by the antibiotic fusidic acid (in red, PDB 4B8F) is in a different conformational state when compared to the structure of EF-G in solution (in green, PDB 1ELO). Upon aligning domains 1 and 2, it is clear that domains 3 to 5 undergo a large hinge-like conformational change. Domain 4 is inserted into the decoding center imposing a conformational constraint on the $30 \mathrm{~S}$ subunit.

(Wintermeyer and Rodnina, 2000, Wintermeyer et al., 2004). This model, in which EF-G imposes a structural constraint on the 16S rRNA and the tRNA, is supported with the data comparing the structure of EF-G in solution and on the ribosome after translocation, suggesting that domains 1 and 2 are largely unchanged while the other ones are reoriented (Figure 1-6) (Agrawal et al., 1998, Wilson and Noller, 1998). Altogether these findings suggest that domains 1 and 2 play a decisive role as a docking site at the ribosome, while the hinge-like movement of domains 3 to 5 plays an active role in translocation. The finding that deletion mutants of EF-G lacking domain 4 and 5 are slower in Pi release and mRNA/tRNA movement, while GTP hydrolysis in unaffected supports this view (Savelsbergh et al., 2000a, Savelsbergh et al., 2003, Rodnina et al., 1997). Further support comes from a study that shows that a mutant EF-G cross-linked between domains 1 and 5 showed is unable to translocate tRNAs (Peske et al., 2000), which suggests that 
the hinge-like motion of EF-G is essential for translocation. Hence, domains 4 and 5 must participate in the unlocking step of elongation, being this the rate limiting step for translocation and $\mathrm{Pi}$ release.

EF-G and the ternary complex EF-Tu-GTP-aa-tRNA bind to the ribosome at the GAC in a very similar manner. This similarity of the binding site led to the suggestion that the relative position of the $L 7 / L 12$ stalk and the $S R L$ selects which elongation factor will bind to the ribosome at each point (Zavialov and Ehrenberg, 2003). In contrast to the rigid $\mathrm{SRL}$, the L7/L12 stalk is flexible; it has been proposed that the different arrangements of the SRL and the L7/L12 stalk with respect to each other alter the affinity for one factor or the other to the ribosome (Sergiev et al., 2005) - however this mechanism is currently still debated. In one previous study no difference regarding EF-G GTP hydrolysis when stimulated by vacant ribosomes, initiation complexes (ribosomes with a tRNA on the P site and vacant $A$ site) and pre-translocation complexes (ribosomes with tRNAs on the A and P site) has been reported (Rodnina et al., 1997) - arguing against this selection model. A different recent study indicated that the selection of factors could be regulated by the dynamic state of the ribosome - suggesting it has different affinities for elongation factors if it is in the ratcheted or unratcheted state (Chen et al., 2013). However, in a previous study with rRNA mutants, there was no difference on GTP hydrolysis by EF-G in ratcheted and unratcheted complexes (Walker et al., 2008). Altogether these studies show that the mechanism by which EF-G and EF-Tu are differentially selected by the ribosome is still unclear and a topic of intense research.

\subsubsection{EF-G as a GTPase molecular motor}

Classical molecular motors couple energy to a directional movement through a conformational change (Keller and Bustamante, 2000). One particularly well-established example is myosin, which does not bind to actin when in complex with ATP. However, upon spontaneous ATP hydrolysis, the myosin-ADP-Pi complex binds to actin in a loose state. Once $\mathrm{Pi}$ is released, the complex rearranges into a tight state generating a power stroke, derived from this structural conformation constraint. Upon structural relaxation, ADP is 
exchanged for ATP and myosin dissociates from actin (Huxley, 2000). Although EF-G and myosin are clearly different, the parallel on the functional cycle is striking. EF-G, just like myosin, presents a delay between GTP hydrolysis and the actual motion. Also, upon $\mathrm{Pi}$ release a structural conformation constraint is relaxed, which, for EF-G is done by a large conformational change of domain 4 , acting as a lever, in contrast to myosin where the power stroke is performed by its head.

Despite these similarities with myosin when put under the lenses of a molecular machine, EF-G is often classified as a switch-type GTPases (Verstraeten et al., 2011, Bourne et al., 1991). However, significant differences make EF-G an exception in this group. Typically, GTPases are active in their GTP-bound form, and upon GTP hydrolysis, conformational changes take place disrupting the interactions of these GTPases with their binding partners. This kind of behavior is largely called a conformational switch - once GDP is exchanged for GTP, the GTPase is 'switched on'; and upon GTP hydrolysis, it is 'switched off' (Bourne et al., 1991, Bourne et al., 1990). One stark example of this is the Ran cycle during nuclear export/import. Ran-GTP has a strong affinity to importins when in the nucleus; upon passage through the nucleopore complex to the cytoplasm, GTP is hydrolyzed, the complex with importin falls apart, and Ran-GDP is shuttled back into the nucleus, where GEFs are present to exchange GDP for GTP thereby restarting the cycle (Gorlich and Kutay, 1999). EF-G behaves in a truly different manner following the mechanism of a molecular motor protein. Upon GTP hydrolysis, instead of having the EF-G-ribosome complex falling apart as in the Ran cycle, it forms a tighter complex where conformational constraints induce other conformational changes on the ribosome, ultimately resulting in tRNA translocation. Further evidence supporting the molecular motor argument comes from studies with EF-G mutants lacking domain 4 (Rodnina et al., 1997), where this 'power stroke' movement cannot be performed (even though single round GTP hydrolysis is unaltered) and the tight complex with the ribosome is kept, preventing EF-G dissociation (Wintermeyer and Rodnina, 2000).

From the discussion of EF-G as a molecular motor, the question about which type of motor it is arises. An understanding of the type of motor is essential since it allows a better insight into the role of GTP hydrolysis. Therefore, is EF-G a molecular motor 
that directly couples the energy from GTP hydrolysis to a motion (such as a deterministic motor (Keller and Bustamante, 2000)) or does it work by biasing the system towards a movement through structural changes caused by GTP hydrolysis (such as in a probabilistic or Brownian motor (Keller and Bustamante, 2000))? The main difference between these two options is how the energy released is coupled to motion - the first states that energy will directly drive motion; the second states that the energy is not directly used, but rather it simply increases the probability of a movement towards one direction to the detriment of another. Should EF-G be a deterministic motor, after GTP hydrolysis and upon Pi release, a motion would always be expected - however this does not hold true for EF-G. As discussed above, studies with antibiotics, EF-G and L7/L12 mutants can affect the movement of the tRNAs and the mRNA or $\mathrm{Pi}$ release without affecting the respective other step (Savelsbergh et al., 2000b, Savelsbergh et al., 2003, Rodnina et al., 1997, Peske et al., 2004). This indicates that these two steps are independent, thus placing EF-G in the category of a probabilistic motor. The observation that translocation also takes place without GTP hydrolysis strengthens this point further (Katunin et al., 2002, Rodnina et al., 1997, Bergemann and Nierhaus, 1983). Hence, it seems that EF-G has two functions during translocation: (i) to provide the conformational rearrangement required to promote ribosome unlocking, the limiting step of tRNA and mRNA movement; (ii) to increase the probability of a forward movement on the unlocked ribosome (Wintermeyer et al., 2004).

\subsubsection{Role of GTP hydrolysis}

All translational GTPases have a highly conserved GTP-binding domain, despite their different functions (Verstraeten et al., 2011). These GTP-binding domains, called G domains, are responsible not only for GTP binding but also for GTP hydrolysis. G domains show three highly conserved functional features: the phosphate-binding loop (P-loop), which binds the nucleotide at its $\alpha$ - and the $\beta$-phosphates; and the switch 1 and switch 2 motifs, which coordinate the $\gamma$-phosphate (Frank et al., 2007). Based on structural studies of the position of switches 1 and 2 and the binding sites of these GTPases on the 
ribosome close to the GAC, it is assumed that the mechanism of GTP hydrolysis is highly conserved among these different factors (Frank et al., 2007). In a different comparative approach, it has been shown that the switch 1 motif is structured in EF-Tu and EF-G in the presence of GTP, but in the presence of GDP, it becomes disordered (Czworkowski and Moore, 1997, Connell et al., 2007, Valle et al., 2003, Vogeley et al., 2001). This indicates that the switch 1 motif plays a critical role in GTP hydrolysis and that the mechanism is very similar in EF-Tu and EF-G - therefore allowing closer comparisons for further understanding. Interestingly, there is a universally conserved histidine in the switch 2 motif that has been shown to play a pivotal role in GTP hydrolysis in EF-Tu (Daviter et al., 2003). Recently, a computer simulation study confirmed the key role of this residue in EF-Tu in GTP hydrolysis, and suggested that the SRL plays a role in correctly positioning this histidine for GTPase activation (Wallin et al., 2013). However, despite the wealth of biochemical and structural information, little is known about the precise mechanism of GTP hydrolysis in EF-G so far. EF-G has no intrinsic GTPase activity, showing that the GAP (GTPase activating protein) function of the ribosome is essential. Although areas of the ribosome, such as the $L 7 / L 12$ stalk and the $S R L$, have already been identified as crucial for GTP hydrolysis (Savelsbergh et al., 2000b, Mohr et al., 2002, Shi et al., 2012, Voorhees et al., 2010), it is not known how this is achieved. Moreover, kinetic studies have shown that the rate of GTP hydrolysis is much faster than that of translocation (Rodnina et al., 1997); however, surprisingly, studies with non-hydrolyzable GTP analogs have shown that translocation takes place, albeit 50 times slower (Katunin et al., 2002). In the light of these two findings, it seems that GTP hydrolysis is not directly coupled to translocation and that instead the energy from GTP hydrolysis accelerates unlocking, the rate-limiting step on translocation. 


\subsection{EF-G and ribosome dynamics during translo- cation}

The role of EF-G during translocation has been studied extensively in the last years, leading to a better mechanistic understanding. EF-G-GTP binds to the A site of a pretranslocation complex (a ribosome with a peptidyl-tRNA at the A site and a deacylated tRNA at the $\mathrm{P}$ site), where GTP hydrolysis takes place rapidly. The details of GTPase activation are not yet fully known, as discussed previously in section 1.3.3. Upon GTP hydrolysis, EF-G-GDP-Pi undergoes a conformational change while the ribosome is rearranged in a process called unlocking. This ribosomal rearrangement precedes the tRNA and mRNA movement itself and therefore is rate-limiting. Upon unlocking, the concerted movement of the mRNA/tRNA complex and Pi release proceeds at random (Shoji et al., 2009, Wintermeyer et al., 2004). This observation was corroborated by different studies using antibiotics, EF-G and L7/L12 mutants, which show that when Pi release or tRNA and mRNA movement are blocked, the other one still proceeds normally (Savelsbergh et al., 2000b, Rodnina et al., 1997, Savelsbergh et al., 2003). Throughout translocation, EF-G undergoes a large hinge-like conformational change on domains 3 to 5 . This leverlike structure formed by these domains would bias the forward tRNA movement, once the ribosome is unlocked (Figure 1-6) (Frank and Agrawal, 2000, Stark et al., 2000, Peske et al., 2000). As soon as $\mathrm{Pi}$ is released, EF-G-GDP undergoes another conformational change that presumably decreases its affinity for the ribosome, leading to its final dissociation from the ribosome alongside the deacylated tRNA at the $E$ site (Wintermeyer et al., 2004, Savelsbergh et al., 2000a).

Changing the focus from EF-G to the ribosome, different dynamics play a role during translocation (Figure 1-3). These processes are hybrid state formation, ribosomal ratcheting and $30 \mathrm{~S}$ subunit head swiveling. Hybrid state formation is the spontaneous movement of the tRNA acceptor arms into the neighboring sites and it is correlated with ribosomal ratcheting (Moazed and Noller, 1989, Dorner et al., 2006, Ermolenko et al., 2007).

Ribosomal ratcheting is the rotational movement of the $30 \mathrm{~S}$ subunit with respect to the $50 \mathrm{~S}$ subunit, which is essential for tRNA and mRNA translocation (Horan and 
Noller, 2007). An obvious assumption from this fact is that there are many structural changes that take place at the intersubunit gap to allow such movement. The proteins S12, S13, L5 and parts of the 23S rRNA seem to be mostly involved in the interactions that take place in the intersubunit gap, holding the subunits together during this highly dynamic process (Cukras et al., 2003, Valle et al., 2003). Cross-linking of proteins of both subunits at the intersubunit gap that prevent subunit rotation lead to ribosomes being unable to translocate (Horan and Noller, 2007), showing that ratcheting is essential for translocation. It is worth noting though that ratcheting and unlocking are different steps - ratcheting is a spontaneous rotation of $30 \mathrm{~S}$ subunit, and it is factor-independent; unlocking is the limiting step prior to translocation and depends on the factor being bound to the ribosome.

The last of these ribosomal movements is the $30 \mathrm{~S}$ subunit head swiveling, which is a ribosomal motion that is loosely coupled to hybrid state formation and ratcheting (Frank et al., 2007, Fischer et al., 2010). The head movement of the 30 S subunit pulls the anticodon stem-loop of the P-site tRNA towards the E site, which, when combined with the action of the L1 stalk, commits the deacylated-tRNA to the E site (Frank et al., 2007, Berk and Cate, 2007, Fei et al., 2008, Trabuco et al., 2010). This major reaction takes place after unlocking when the conformational change imposed by domain 4 of EF-G on the ribosome drives the detachment of the mRNA/tRNA complex from the decoding center on the $30 \mathrm{~S}$ subunit (Stark et al., 2000). Although the mRNA/tRNA complex is free from the body of the $30 \mathrm{~S}$ subunit, the complex remains strongly bound to the head domain of the 30 S subunit (Ratje et al., 2010). In this moment, head swiveling is thought to play the crucial role, by moving the mRNA towards the $E$ site by one codon while maintaining the mRNA/tRNA interactions. Structural studies with the translocation inhibitor spectinomycin show that when $30 \mathrm{~S}$ subunit head swiveling is blocked, tRNA movement at the 50 S subunit is unchanged (Pan et al., 2007, Borovinskaya et al., 2007) - this suggests that head swiveling plays an important role in the full translocation step. Finally, it is believed that a combination of the movements of a back-rotation of the head of $30 \mathrm{~S}$ subunit, the reverse-ratcheting of the ribosome and the relaxation of the structural constraint imposed by EF-G are responsible for loosening the affinity of the $30 \mathrm{~S}$ subunit 
head to this mRNA/tRNA complex and to the dissociation of EF-G from the ribosome (Frank et al., 2007).

In the light of all these structural and kinetic studies, a model has been proposed in which GTP hydrolysis and the subsequent Pi release promote conformational changes of EF-G that lead to structural rearrangements on the ribosome.

\subsubsection{Classical and hybrid states}

The ribosome and EF-G are not the only dynamic part involved in translation; tRNAs are also very active, and their spontaneous movements between their different binding sites on the ribosome seem to follow their intrinsic thermal fluctuations (Moazed and Noller, 1989). This spontaneous movement of the tRNAs after peptide bond formation leads to at least two very distinct states: a classical and a hybrid state. In the classical state, the tRNAs are positioned in a way that the acceptor arm of the tRNA on the 50S subunit is positioned at the $A$ or the $P$ site, while on the $30 S$ subunit their anticodon stem loop counterpart is also at the $A$ or $P$ site $(A / A$ and $P / P)$, respectively (Moazed and Noller, 1989). In the hybrid state, there is a movement on the acceptor arms of the tRNAs on the 50S subunit towards the neighboring site while the anticodon stem loop does not move leading to $A / P$ and $P / E$ configurations. Since the hybrid state $A / P$ is not puromycin-reactive (Moazed and Noller, 1989, Sharma et al., 2004, Semenkov et al., 2000), the acceptor arm of the A site tRNA is not a true $P$ site conformation. The transitions between classical and hybrid states are spontaneous and fast (up to 5 per second, much faster than spontaneous translocation, but slower than factor-dependent translocation) (Blanchard et al., 2004, Munro et al., 2007). Structural studies have also pointed out that the $30 \mathrm{~S}$ subunit ratchet motion correlates with hybrid state formation in the presence of EF-G (Frank et al., 2007). Taken together, this indicates that EF-G stabilizes the ratcheted state of the ribosome, as well as the hybrid state of the tRNAs - states that would otherwise fluctuate dynamically between each other (Fischer et al., 2010). Despite the recent focus on these transitions and states have in many studies, their roles are still heavily debated. One of the open questions is whether EF-G stabilizes 
the hybrid state upon binding or whether EF-G preferably binds to the ribosome when the tRNAs are in the hybrid state. In summary, the role of hybrid state formation is still debated as well as the significance of these transitions and their on EF-G.

\subsubsection{Synchronous translocation requires GTP hydrolysis}

Another aspect of tRNAs dynamics that is still in discussion is the synchronicity of translocation. It is known that hybrid state formation occurs independently of translocation and that the positions assumed by the tRNA acceptor arms on the $50 \mathrm{~S}$ subunit are different from those reached after the full translocation (Moazed and Noller, 1989). Moreover, the ratchet movement of the $30 S$ subunit could allow a larger degree of freedom with respect to the mRNA/tRNA complex in the decoding center. These two spontaneous and

independent movements lead to the speculation of a two-step mechanism of translocation, with the first step taking place on the $50 \mathrm{~S}$ subunit, followed by the translocation of the mRNA/tRNA complex on the $30 \mathrm{~S}$ subunit (Agirrezabala and Frank, 2009). Since no direct evidence to support this mechanism has been provided, the timing of the tRNA translocation of the two ribosomal subunits is still unknown.

\subsection{L7/L12 stalk}

The L7/L12 stalk is a large and flexible tentacle-like protrusion of the $50 \mathrm{~S}$ subunit (Figure 1-7) (Diaconu et al., 2005). It is believed to play a crucial role in factor recruitment and GTPase activation (Diaconu et al., 2005, Kischa et al., 1971). In E. coli the stalk itself is composed of L10, L11 and 4 copies of L7/L12 dimers (Gudkov, 1997), however, the number of copies varies among different eubacterial species (Davydov et al., 2013). L7 and L12 are variants of the same protein, L7 being the N-acetylated form (Gudkov, 1997, Savelsbergh et al., 2000b). L10 and L11 interact with parts of 23S rRNA forming the basis of the stalk. The C-terminal region of L10 interacts with the $\mathrm{N}$-terminal domains of L7/L12 dimers, connecting these flexible proteins to the ribosome (Diaconu et al., 2005).

L7/L12 itself is structurally divided into 3 parts (Figure 1-7): the N-terminal part, which binds to the ribosome via L10, the C-terminal domain, which interacts with trans- 


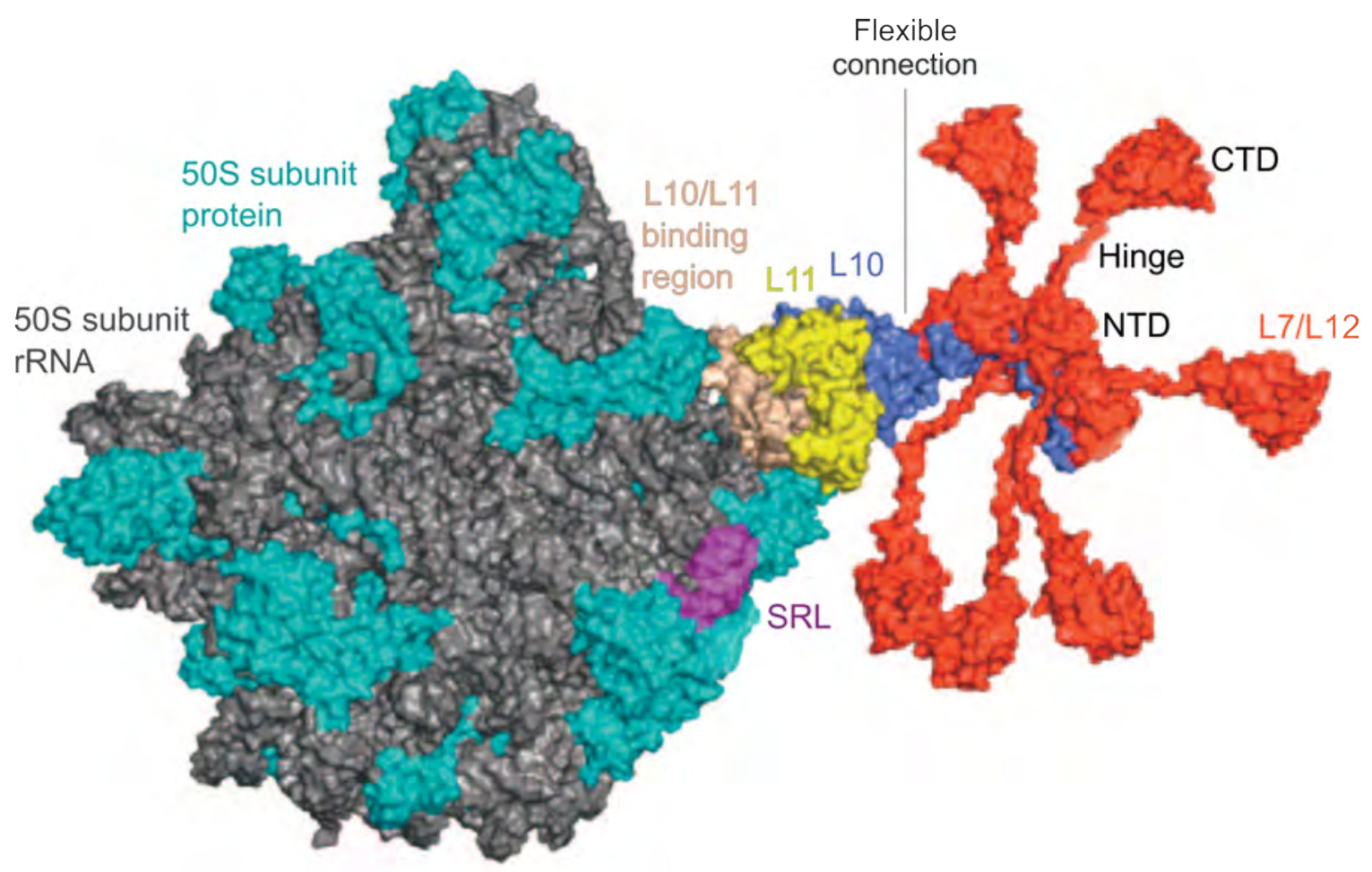

Figure 2-7: L7/L12 stalk. Structural model of the Thermus termophilus L7/L12 stalk on the 50S subunit. This model was obtained combining X-ray, cryo-EM and nuclear magnetic resonance (NMR) structures of the ribosome, L11 (yellow), L10 (blue), and the N- and C-termini of L7/L12 (red). Image modified from Diaconu and colleagues (Diaconu et al., 2005).

lation factors, and a highly flexible hinge region connecting the $\mathrm{N}$ - and $\mathrm{C}$-terminal domains (Gudkov, 1997, Gudkov et al., 1991, Liljas and Gudkov, 1987, Kopke et al., 1992, Agthoven et al., 1975, Wahl et al., 2000). These flexible hinges are necessary for factor recruitment - allowing EF-Tu and EF-G to bind to the ribosome faster than what would be expected for simple diffusion (Savelsbergh et al., 2003, Rodnina et al., 1996). Deletion and rescue studies have been done with the hinge domain showing that, despite its presence being essential for protein recruitment and GTPase activation, its composition is irrelevant (Bubunenko et al., 1992, Oleinikov et al., 1993). Interestingly, it seems that all translational GTPases interact with L7/L12 at the same area of the C-terminal domain, based on studies with multiple alignments and monoclonal antibodies against specific C-terminal regions (Sommer et al., 1985, Leijonmarck and Liljas, 1987), indicating an evolutionary conserved binding domain. 


\subsubsection{Factor recruitment}

The role of the L7/L12 stalk has puzzled the scientific community for a long time, and although a lot is known about its function, the underlying question of how L7/L12 recruits factors and promotes GTP hydrolysis is still open. C-terminal deletions on L7/L12 produced virtually inactive ribosomes (Kischa et al., 1971, Hamel et al., 1972), mainly due to its decreased ability to interact with elongation factors - moreover, effects on initiation and termination factors have also been reported (Kay et al., 1973, Brot et al., 1974, Fakunding et al., 1973). Proteolysis studies have shown that L7/L12 interact with elongation factors differently after GTP hydrolysis (Gudkov, 1997, Gudkov and Bubunenko, 1989, Gudkov and Gongadze, 1984), suggesting that this stalk might have a docking role, as well as regulating factor occupancy of the A-site. As the L7/L12 stalk is composed of many copies of L7/L12 (ranging from 4 up to 8 copies per ribosome) (Davydov et al., 2013), it suggests that its C-terminal domain fishes for translation factors and places them at the ribosomal binding site. The fact that there is more than one component doing this task increases the likelihood of this event to be successful (Diaconu et al., 2005, Rodnina et al., 1996). The length and the flexibility of the hinge are also crucial for the acting range.

\subsubsection{GTPase activation}

Perhaps even more important than factor recruitment is the role that the L7/L12 stalk plays in modulating GTPase activity. It has long been known that the stalk is important for the stimulation of ribosome-activated GTP hydrolysis of translation elongation factors (Diaconu et al., 2005), but the underlying mechanism for activation is not yet fully understood. Surprisingly, isolated L7/L12 can stimulate GTP hydrolysis on EF-G even though, with rates much lower than those measured in the presence of the entire ribosome (Savelsbergh et al., 2000b), indicating that some of the residues involved in GTPase activation are present on the binding interface between L7/L12 and EF-G. This evidence strongly suggests that the L7/L12 stalk, together with the SRL, contribute to the GTPase activation of EF-G. The mechanism by which $L 7 / \mathrm{L} 12$ regulates the GTPase acti- 
vation is suggested to be through structural changes at the active site by direct contacts reorganizing the catalytic residues with the GTPases or by donating additional catalytic groups in trans (allostery) (Wahl and Moller, 2002, Diaconu et al., 2005). However, mutation analysis studies in EF-Tu and EF-G showed that all the conserved residues mutated only affected the binding affinity of elongation factors to the ribosome, while the GTPase activity remained unchanged (Diaconu et al., 2005, Savelsbergh et al., 2005, Kothe et al., 2004) indicating that any role played by L7/L12 in GTPase activation might be indirect, most likely through rearrangements of the SRL and by stabilization of the GTPase transition state.

The role of $\mathrm{L} 7 / \mathrm{L} 12$ can be summarized as being $\mathrm{L} 7 / \mathrm{L} 12$ involved in translation factor binding and activation. Upon the initial contact with the C-terminal domain of L7/L12, the translation factors are able to interact with the ribosome GTPase activation domain with the help of the flexible hinge.

\subsubsection{The stalk and translocation}

There is a lot of speculation on how the L7/L12 stalk recruits factors and how it acts as a GTPase activator, however, experimental evidence that would allow a comprehensive understanding of the process is still missing. So far, it has been shown that when the stalk is removed (Kischa et al., 1971, Hamel et al., 1972) or when conserved residues at the C-terminus are mutated, translocation is slowed down (Savelsbergh et al., 2005, Diaconu et al., 2005), most likely due to poor factor binding. However details on how L7/L12 recruits factors and how GTPases are activated are still unknown and are currently investigated. 


\section{3}

\section{Material and Methods}

\subsection{Buffers and reagents}

Buffer A: $50 \mathrm{mM}$ Tris- $\mathrm{HCl}, \mathrm{pH}$ 7.5, $70 \mathrm{mM} \mathrm{NH}_{4} \mathrm{Cl}, 30 \mathrm{mM} \mathrm{KCl}$ and $7 \mathrm{mM} \mathrm{MgCl} 2$. Chemicals were from Roche Molecular Biochemicals, Sigma Aldrich, or Merck. Radioactive compounds were from Hartmann Analytic

\subsection{Ribosomes, mRNAs, tRNAs, and translation factors}

Ribosomes from E. coli MRE 600, $\mathrm{f}\left[{ }^{3} \mathrm{H}\right]$ Met-tRNA $^{f M e t}, \mathrm{f}\left[{ }^{3} \mathrm{H}\right]$ Met-tRNA $^{f M e t}(\mathrm{Flu}),\left[{ }^{14} \mathrm{C}\right]$ PhetRNA $^{\text {Phe }}, \mathrm{EF}-\mathrm{Tu}$, and initiation factors were prepared as described (Rodnina et al., 1995, Savelsbergh et al., 2003, Peske et al., 2004, Milon et al., 2007). Proflavin-labeled tRNA ${ }^{P h e}$ (yeast) and tRNA ${ }^{f M e t}$ (E. coli) were prepared according to published protocols (Wintermeyer et al., 1979a, Wintermeyer et al., 1979b). The mRNA constructs (IBA, Göttingen) were 30 to 33-nucleotides long and contained one (MF-mRNA) or two (MFF-mRNA) Phe codons or one (MV-mRNA) valine codon following the AUG codon. 


\subsection{BODIPY-Met-tRNA ${ }^{f M e t}$}

Met-tRNA ${ }^{f M e t}$ was prepared and purified by HPLC as described (Milon et al., 2007) except that $\mathrm{N}^{10}$-formyltetrahydrofolate was omitted. Modification of [ $\left.{ }^{3} \mathrm{H}\right]$ Met-tRNAfMet at the $\alpha$-amino group with BODIPY-FL sulfosuccinimidyl ester (Bpy-SSE; Invitrogen, D6140) (Gite et al., 2000) was carried out by incubating [ $\left.{ }^{3} \mathrm{H}\right]$ Met-tRNAfMet $(30 \mu \mathrm{M})$ with a 130-fold excess of Bpy-SSE (4 mM) in $20 \mathrm{mM}$ HEPES buffer ( $\mathrm{pH} 8.5)$ for 4 min at $0{ }^{\circ} \mathrm{C}$. The reaction was stopped by adding potassium acetate $\mathrm{pH} 5$ to $0.2 \mathrm{M}$, and tRNA was precipitated by adding 2.5 volumes of ice-cold ethanol. Excess dye was removed by four additional precipitation steps. The resulting tRNA pellet was dried, dissolved in $\mathrm{H}_{2} \mathrm{O}$ and stored at $-80^{\circ} \mathrm{C}$. The concentration of tRNA was determined by radioactive counting and the extent of modification was assessed photometrically, using extinction coefficients of $75,000 \mathrm{M}^{-1} \mathrm{~cm}^{-1}$ for Bpy $(505 \mathrm{~nm})$, and $575,000 \mathrm{M}^{-1} \mathrm{~cm}^{-1}$ for tRNA (260 nm), yielding labeling efficiencies of $80 \%$. Bpy- $\left[{ }^{3} \mathrm{H}\right]$ Met-tRNA ${ }^{f M e t}$ was fully active in initiation complex formation. Protocol developed by Wolf Holtkamp as described in Holtkamp et al. 2013.

\subsection{Expression and purification of EF-G and EF-G mutants}

\subsubsection{Wild type and H91A mutation}

The gene coding for EF-G was cloned into pET24a(+) (Novagen) or pTXB1 (NEB). The pET24EF-G plasmid was used for the expression of wt EF-G with an N-terminal His tag. The H91A mutation was introduced into both vectors using the QuikChange protocol. pTXB1EF-G(H91A) was used for expression of EF-G(H91A) with an N-terminal intein cleavage site, a chitin-binding domain, and an additional His tag. Wt EF-G was overexpressed in BL21(DE3) and EF-G(H91A) in BL21(DE3)pLysS. Cells were grown in LB medium supplemented with kanamycin $(30 \mu \mathrm{g} / \mathrm{ml})(\mathrm{wt})$ or with ampicillin $(50 \mu \mathrm{g} / \mathrm{ml})$ $(\mathrm{H} 91 \mathrm{~A})$ at $37^{\circ} \mathrm{C}$; expression was induced by the addition of IPTG $(1 \mathrm{mM})$, and cultures were grown further for $4 \mathrm{~h}$. Cells were harvested and pellets were resuspended in Protino 
buffer $(20 \mathrm{mM}$ TRIS-HCl, pH 7.4, $300 \mathrm{mM} \mathrm{NaCl}$ ) with the addition of Complete Protease Inhibitor (Roche) and a trace of DNasel. Cells were opened using an Emulsiflex apparatus (Avestin), and the extract was centrifuged for $30 \mathrm{~min}$ at $30,000 \mathrm{~g}$. The supernatant was applied to a Protino gravity-flow column (Macherey-Nagel) for affinity purification using the His tag. The column was washed with Protino buffer and the protein was eluted with Protino buffer containing $250 \mathrm{mM}$ imidazole. The eluted wt protein was concentrated and the buffer was exchanged to 2x buffer A by membrane filtration (Vivaspin 30,000); for storage, one volume of glycerol was added. For EF-G(H91A), the eluate was dialyzed twice against cleavage buffer (20 mM TRIS- $\mathrm{HCl}, \mathrm{pH} 8.5,500 \mathrm{mM} \mathrm{NaCl}$ ), $50 \mathrm{mM}$ of sodium 2mercaptosulfonate (MesNa) were added and the tags were removed by intein cleavage at $4{ }^{\circ} \mathrm{C}$ for $16 \mathrm{~h}$. The proteins were reapplied to a Protino gravity-flow column and the flowthrough was collected. The proteins were concentrated and the buffer was exchanged to 2x buffer A by membrane filtration (Vivaspin 30,000); for storage, one volume of glycerol was added. EF-G concentrations were determined by spectrophotometry at $280 \mathrm{~nm}$ using an extinction coefficient of $64,300 \mathrm{M}^{-1} \mathrm{~cm}^{-1}$.

\subsubsection{H583K and $\Delta 4 / 5$ mutation}

The H583K mutation was introduced in the pET24EF-G plasmid using the QuikChange protocol. EF-G $(\Delta 4 / 5)$ were expressed using plasmid pTXB1. With the gene coding for EF-G cloned into pTXB1, the EF-G(4/5) construct was produced by deletion of amino acids R475 to K704 using the Phusion polymerase deletion protocol (NEB). EF-G mutants were overexpressed in BL21(DE3) and purified as described above. The concentration was determined either by spectrophotometry at $280 \mathrm{~nm}$, using an extinction coefficient of $64,300 \mathrm{M}^{-1} \mathrm{~cm}^{-1}$ or by SDS-PAGE and densitometry using a reference protein.

\subsection{Growth curves}

BL21(DE3)pLysS cells were transformed with pET24EF-G and pET24EF-G(H91A) and plated on LB agar with $30 \mu \mathrm{g} / \mathrm{ml}$ kanamycin and $1 \%$ glucose and grown overnight. Single colonies were picked and overnight cultures were inoculated. Cells were pelleted, resus- 
pended in fresh medium, expression cultures were inoculated to an $\mathrm{OD}_{600}$ of 0.1 , and grown at $37{ }^{\circ} \mathrm{C}$. After 100 min expression was induced by addition of $1 \mathrm{mM}$ IPTG and the cultures were grown for another $3 \mathrm{~h}$.

\subsection{Labeling of $30 \mathrm{~S}$ subunits}

The gene coding for ribosomal protein S13 (rpsM) was cloned into a pET24a vector. The single native Cys85 was replaced with Ser and Cys was engineered at position Pro112. Expression, purification and refolding of S13 were performed essentially as described (Hickerson et al., 2005). Labeling with Atto540Q was performed under denaturing conditions with 10-fold molar excess of dye for $2 \mathrm{~h}$ at $25^{\circ} \mathrm{C}$ in buffer ( $6 \mathrm{M}$ urea, $50 \mathrm{mM}$ HEPES, $\mathrm{pH} 7.1,300 \mathrm{mM} \mathrm{KCl}, 10 \%$ glycerol). The reaction was stopped by the addition of $6 \mathrm{mM}$ 2-mercaptoethanol, excess dye was removed by cation exchange chromatography (HiTrap SP HP column, GE-Healthcare). The degree of labeling was $100 \%$, as determined spectrophotometrically. Reconstitution of purified $30 \mathrm{~S} \Delta \mathrm{S} 13$ ribosomal subunits (from E. coli strain MG $1655 \Delta$ rpsM::kan, kindly provided by Rachel Green) was performed in buffer (50 mM HEPES, pH 7.5, $400 \mathrm{mM} \mathrm{KCl}, 20 \mathrm{mM} \mathrm{MgCl}$, $6 \mathrm{mM}$ 2-mercaptoethanol) with a 1.75-fold molar excess of Atto540Q-labeled protein S13.45 The mixture was incubated for $60 \mathrm{~min}$ at $47^{\circ} \mathrm{C}$ in the dark. 30S subunits were purified by centrifugation through a $30 \%$ sucrose cushion in the same buffer. Pellets were resuspended in buffer A. $30 \mathrm{~S}$ subunits were labeled to $90-100 \%$, as determined by spectrophotometric analysis. Protocol stabilished and carried out by Riccardo Belardinelli.

\subsection{Ribosome complexes}

To prepare pre-translocation complex, ribosomes $(0.6-1 \mu \mathrm{M} 70 \mathrm{~S}$ or labeled $30 \mathrm{~S}$ together with a 1.5-fold excess of 50S subunits) were incubated with a 3-fold excess of mRNA and a 1.5 to 2 -fold excess each of IF1, IF2, IF3, and 1.5-fold excess of f[ $\left.{ }^{3} \mathrm{H}\right]$ Met-tRNA $^{\text {fMet }}$, $\mathrm{f}\left[{ }^{3} \mathrm{H}\right]$ Met-tRNA ${ }^{f M e t}(\mathrm{Flu})$ or Bpy- $\left[{ }^{3} \mathrm{H}\right]$ Met-tRNA ${ }^{f M e t}$ in buffer A containing 1 mM GTP for 30 min at $37{ }^{\circ} \mathrm{C}$. Ternary complex EF-Tu- $\left[{ }^{14} \mathrm{C}\right]$ Phe-tRNA ${ }^{\text {Phe }}$-GTP was prepared by incu- 
bating EF-Tu (2-fold excess over Phe-tRNA ${ }^{\text {Phe }}$ ) with GTP (1 mM), phosphoenolpyruvate $(3 \mathrm{mM})$, and pyruvate kinase $(0.5 \mu \mathrm{g} / \mathrm{ml})$ for $15 \mathrm{~min}$ at $37{ }^{\circ} \mathrm{C}$ and then with $\left[{ }^{14} \mathrm{C}\right]$ PhetRNA $^{\text {Phe }}$ (2-fold excess over ribosomes) for an additional min. Equal volumes of initiation complex and ternary complex were mixed and incubated for $1 \mathrm{~min}$ at $37^{\circ} \mathrm{C}$. Pretranslocation complexes used for stopped-flow experiments were purified by centrifugation through a $1.1 \mathrm{M}$ sucrose cushion in buffer $\mathrm{A}$ with $20 \mathrm{mM} \mathrm{MgCl}$. Pellets were dissolved in buffer $A$ with $20 \mathrm{mM} \mathrm{MgCl}_{2}$ and tRNA binding was verified by nitrocellulose filtration.

To form ternary complex containing $\left[{ }^{14} \mathrm{C}\right]$ Phe-tRNA ${ }^{\text {Phe }}$ (Prf) or $\left[{ }^{14} \mathrm{C}\right]$ Val-tRNA ${ }^{\text {Val }}$ (Prf), deacylated proflavinated tRNA $(25 \mu \mathrm{M})$ was incubated in buffer $\mathrm{A}$ with the respecive purified aa-tRNA-synthetase, GTP $(1 \mathrm{mM})$, ATP $(3 \mathrm{mM})$, DTT $(1 \mathrm{mM}),\left[{ }^{14} \mathrm{C}\right]$ Val or $\left[{ }^{14} \mathrm{C}\right]$ Phe $(45 \mu \mathrm{M})$, pyruvate kinase $(0.1 \mathrm{mg} / \mathrm{ml})$ and EF-Tu $(48 \mu \mathrm{M})$ for $30 \mathrm{~min}$ at 37 ${ }^{\circ} \mathrm{C}$. Ternary complex was purified by size-exclusion chromatography on a Biosuite $250 \mathrm{HR}$ column using an Alliance HPLC system (Waters). Fractions containing ternary complex were pooled, mixed with initiation complex and incubated for $1 \mathrm{~min}$. Pre-translocation complexes used for stopped-flow experiments were purified by centrifugation through a 1.1 $\mathrm{M}$ sucrose cushion in buffer $\mathrm{A}$ with $21 \mathrm{mM} \mathrm{MgCl}$. Pellets were dissolved in buffer $\mathrm{A}$ with $21 \mathrm{mM} \mathrm{MgCl} 2$ and tRNA binding was verified by nitrocellulose filtration. The functional activity of pre-translocation complexes was tested by the puromycin assay (Rodnina et al., 1999). For the kinetic experiments, the concentration of $\mathrm{MgCl}_{2}$ was lowered to $7 \mathrm{mM}$.

\subsection{Turnover GTP hydrolysis}

Vacant ribosomes $(0.5 \mu \mathrm{M})$ were mixed with EF-G $(0.5 \mu \mathrm{M})$ in buffer A with $1 \mathrm{mM}$ GTP and trace amounts of $\left[\gamma_{-}{ }^{32} \mathrm{P}\right] \mathrm{GTP}$ at $20{ }^{\circ} \mathrm{C}$. Samples were taken and quenched with one volume of $40 \%$ formic acid. Samples were analyzed by TLC (Polygram CEL 300, Macherey-Nagel) using $0.5 \mathrm{M}$ potassium phosphate ( $\mathrm{pH} 3.5$ ) running buffer. Radioactivity was detected using a phosphoimager system. 


\subsection{Puromycin reaction}

Translocation was tested using the puromycin assay. Pre-translocation complexes $(0.1$ $\mu \mathrm{M})$ were incubated with various amount of $\mathrm{EF}-\mathrm{G}$ or $\mathrm{EF}-\mathrm{G}(\mathrm{H} 91 \mathrm{~A})$ for 3 min at $20{ }^{\circ} \mathrm{C}$ in buffer A. Samples were then reacted with puromycin $(1 \mathrm{mM})$ for $10 \mathrm{~s}$ before being quenched with $1.5 \mathrm{M}$ sodium acetate saturated with $\mathrm{MgSO}_{4}$. $\mathrm{f}\left[{ }^{3} \mathrm{H}\right] \mathrm{Met}\left[{ }^{14} \mathrm{C}\right] \mathrm{Phe}$-puromycin was extracted with ethyl acetate and quantified by radioactivity counting.

\subsection{Rapid kinetics methods}

\subsubsection{Single-round GTP hydrolysis}

Single-round GTP hydrolysis was measured in buffer $\mathrm{A}$ at $20{ }^{\circ} \mathrm{C}$ in a quench-flow device (KinTek Laboratories, Inc.) by rapidly mixing equal volumes of vacant ribosomes $(1 \mu \mathrm{M})$ and mant-GTP $(50 \mu \mathrm{M})$ (Jena Biosciences) with EF-G (wt or H91A) $(5 \mu \mathrm{M})$ and mantGTP $(50 \mu \mathrm{M})$. Samples were quenched with $40 \%$ formic acid. Following neutralization by $\mathrm{KOH}$, samples were analyzed by HPLC monitoring mant fluorescence. The rates of GTP hydrolysis obtained by this method are identical to those measured with [ $\left.{ }^{32} \mathrm{P}\right] \mathrm{GTP}$ (Liudmila Filonava, Pohl Milon and Marina V. Rodnina, manuscript in preparation).

\subsubsection{Pi release}

Pi release was measured in a stopped-flow apparatus (Applied Photophysics) (Brune et al., 1994, Savelsbergh et al., 2003). Vacant ribosomes $(0.5 \mu \mathrm{M})$ were rapidly mixed with EF-G $(0.5 \mu \mathrm{M})$ in buffer A, GTP $(1 \mathrm{mM})$, and MDCC-labeled phosphate-binding protein (PBP) $(2.5 \mu \mathrm{M})$, both pre-incubated with Pi mop $(0.1 \mathrm{U} / \mathrm{ml}$ PNPase, $0.2 \mu \mathrm{M} 7$ methylguanosine). MDCC fluorescence was excited at $425 \mathrm{~nm}$ and measured after passing a KV450. 


\subsubsection{Single-round translocation}

Rapid kinetics of translocation was measured in buffer A containing $1 \mathrm{mM} \mathrm{GTP}$ at $37{ }^{\circ} \mathrm{C}$ by stopped-flow (Rodnina et al., 1997, Savelsbergh et al., 2003, Savelsbergh et al., 2005). Alx488, Prf, Flu and Bpy fluorescence was excited at $470 \mathrm{~nm}$ and detected after passing a KV500 cut-off filter (Schott). Alx405 fluorescence was excited at $400 \mathrm{nM}$ and measured after passing a KV418 cut-off filter (Schott). Equal volumes of pre-translocation complexes $(80 \mathrm{nM})$ were rapidly mixed with EF-G or EF-G(H91A) at different concentrations as indicated. Experiments with EF-G mutants ( $\mathrm{H} 583 \mathrm{~K}$ and $\Delta 4 / 5)$ were performed at saturating EF-G concentration $(4 \mu \mathrm{M})$.

\subsubsection{EF-G ribosome complex formation and dissociation}

EF-G-ribosome complex formation and dissociation was monitored by the fluorescence of mant-labeled nucleotides. Ribosomes $(0.5 \mu \mathrm{M})$ were rapidly mixed with $0.5 \mu \mathrm{M} E \mathrm{EF}-\mathrm{G}$ and mant-GTP or mant-GDP $(5 \mu \mathrm{M})$ in buffer $\mathrm{A}$ at $37^{\circ} \mathrm{C}$ in a stopped-flow apparatus. Complex formation was monitored by mant fluorescence excited by FRET from tryptophan excited at $290 \mathrm{~nm}$ after passing a KV408 filter (Schott). For chase experiments, preformed EF-G-mant-nuclotide-ribosome complexes $(0.5 \mu \mathrm{M})$ were rapidly mixed with unlabeled GTP or GDP $(1 \mathrm{mM})$ in buffer $A$ at $37^{\circ} \mathrm{C}$.

\subsection{EF-G-ribosome pull-down assay}

Pre-translocation complexes $(0.1 \mu \mathrm{M})$ and EF-G (wt or H91A) $(0.2 \mu \mathrm{M})$ were incubated in buffer A with GTP $(1 \mathrm{mM})$ for $5 \mathrm{~min}$ at $20{ }^{\circ} \mathrm{C}$. Samples were centrifuged through $400 \mu \mathrm{l}$ sucrose cushions ( $40 \%$ in buffer A with $20 \mathrm{mM} \mathrm{MgCl} 2$ ) at $259,000 \mathrm{~g}$ for $2 \mathrm{~h}$. Pellets were resuspended in buffer $A$ and concentrations were measured photometrically at $260 \mathrm{~nm}$. Samples $(50 \mathrm{pmol})$ were analyzed on a pre-casted 4-20\% SDS polyacrylamide gradient gel (Serva). Pull-down experiments with vacant ribosomes were performed with mant-GTP/GDP $(5 \mu \mathrm{M})$ to allow comparisons to EF-G ribosome complex formation experiments. 


\subsection{Tripeptide formation}

Initiation complexes $(0.5 \mu \mathrm{M})$ with $\left.\mathrm{f}^{3} \mathrm{H}\right] \mathrm{Met}^{\mathrm{N}} \mathrm{tRNA} \mathrm{A}^{f M e t}$ in the $\mathrm{P}$ site and programed with an mRNA coding for fMetPhePhe were incubated with wt EF-G or EF-G(H91A) $(1 \mu \mathrm{M})$ for $1 \mathrm{~min}$ in buffer $\mathrm{A}$ at $37{ }^{\circ} \mathrm{C}$ before EF-Tu- $\left[{ }^{14} \mathrm{C}\right]$ Phe-tRNA ${ }^{\text {Phe }}$-GTP $(2 \mu \mathrm{M})$ was added. After $2 \mathrm{~min}$ at $37{ }^{\circ} \mathrm{C}$ samples were quenched with $1 / 10$ volume of $0.5 \mathrm{M} \mathrm{KOH}$ and hydrolyzed for $30 \mathrm{~min}$ at $37^{\circ} \mathrm{C}$. Samples were neutralized with $1 / 10$ volume of acetic acid and peptides were analyzed by HPLC (Spiegel et al. 2007).

\subsection{Nucleotide binding}

To minimize protein adsorption, cuvettes were treated with a solution of BSA $(1 \mathrm{mg} / \mathrm{ml})$ in buffer $\mathrm{A}$ for $15 \mathrm{~min}$ and rinsed with buffer $\mathrm{A}$. EF-G $(1 \mu \mathrm{M})$ was titrated with either GTP or mant-GDP in buffer $\mathrm{A}$ at $37^{\circ} \mathrm{C}$. After each nucleotide addition the sample was equilibrated for 5 min before a reading was taken. Tryptophan fluorescence (GTP) was excited at $290 \mathrm{~nm}$ and emission was measured at $350 \mathrm{~nm}$. Mant-GDP fluorescence was excited by FRET from tryptophan as above and measured at $445 \mathrm{~nm}$. Titration curves were evaluated using a quadratic equation. 


\section{4}

\section{Results}

\subsection{Construction of the GTPase-deficient EF-G mu- tant}

The conserved histidine at position 91 in the G domain of E. coli EF-G (Figure 4-1) was replaced with alanine. Cells transformed with the pET24a plasmid containing the gene coding for mutated EF-G were impaired in growth after induction with IPTG (Figure 4-1), indicating that the expression of inactive EF-G conferred a dominant lethal phenotype. To avoid the growth inhibition, we designed a construct in which the gene coding for mutant EF-G contained a C-terminal intein domain followed by a chitin-binding domain and a His tag. This construct allowed the expression of EF-G(H91A) without any detrimental effect on cell growth.

The mutated factor was inactive in GTP hydrolysis on the ribosome under conditions of multiple turnover, i.e., with catalytic amounts of factor relative to ribosomes, as monitored by TLC with $\left[\gamma_{-}{ }^{32} \mathrm{P}\right] \mathrm{GTP}$ (Figure $\left.4-2\right)$ or the release of inorganic phosphate (Pi) monitored by the fluorescence of MDCC-labeled phosphate-binding protein (Figure 4-3). The inhibition of the turnover reaction was caused by the inability of EF-G(H91A) to hydrolyze GTP, rather than its impaired ability to dissociate from the ribosome, as verified by the lack of GTP hydrolysis at single-round GTPase conditions, which reflects the bona fide ability of the factor to cleave GTP. The time course of single-round hydrolysis was 


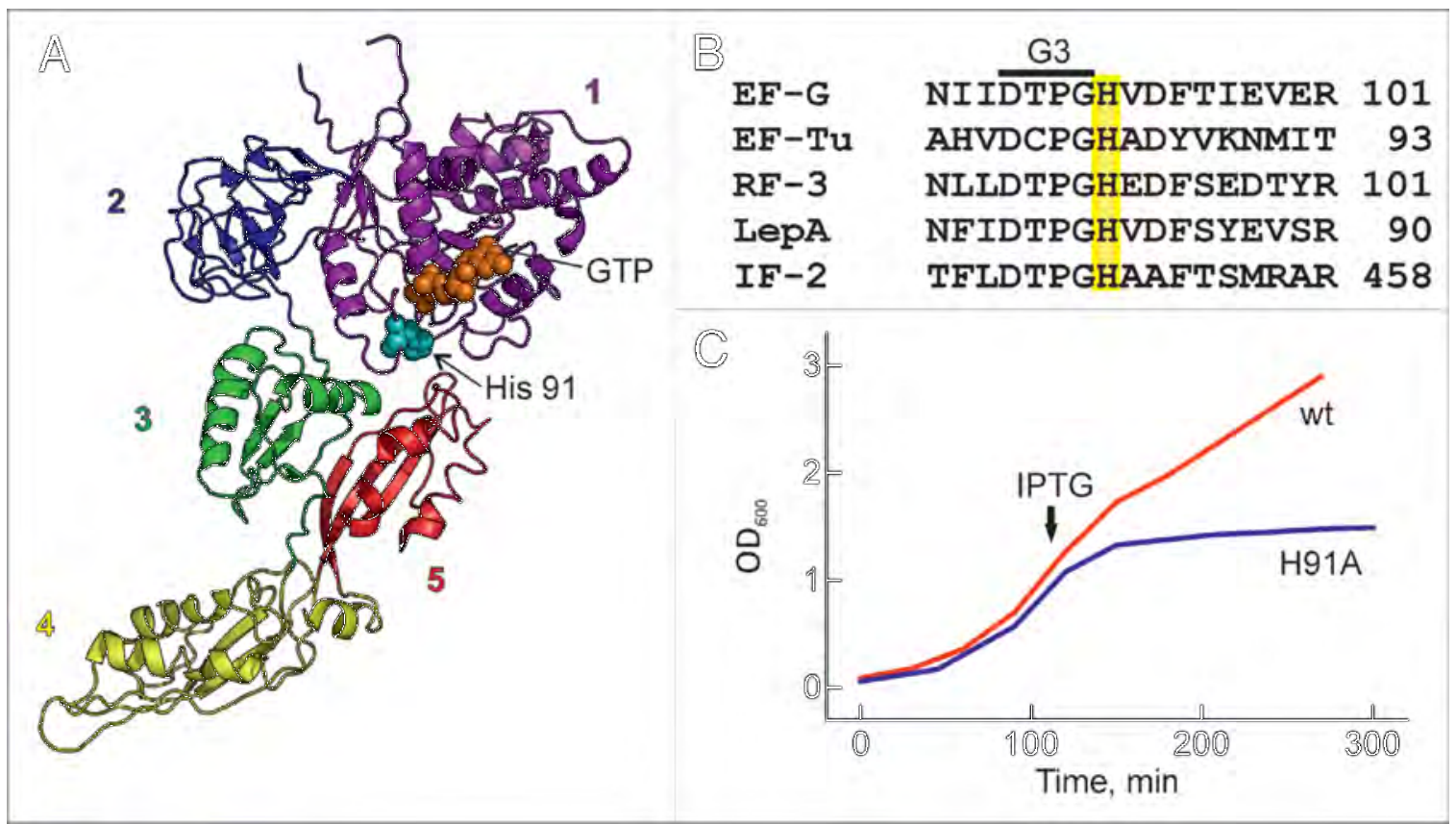

Figure 4-1: The H91A mutation in EF-G. (A) Structure of EF-G (PDB 2WRI). The domains of EF-G are numbered 1-5 and color-coded. Residue His91 in domain 1 is in cyan, GTP (orange) was modeled instead of GDP. (B) Sequence conservation within the $\mathrm{G}$ domain of translational GTPases. The conserved histidine residue (His91 in EF-G, His84 in EF-Tu) following the G3 motif DXXG (bar) is indicated. (C) Growth curves of E. coli BL21(DE3)pLysS cells transformed with the pET24a plasmid containing the gene coding for either wt EF-G or EF-G(H91A). The induction of expression by IPTG is indicated (arrow).

measured by quench-flow at an excess of factor over ribosomes, using a fluorescent GTP derivative, mant-GTP. The reaction was monitored by the increasing ratio of mant-GDP to mant-GTP, as determined by HPLC analysis (Figure 4-4). In that assay, the wild-type (wt) factor hydrolyzed close to one equivalent of GTP per ribosome during a rapid burst phase which was followed by a slower turnover reaction, whereas the mutant factor was completely inactive. However, equilibrium titrations with GTP, measuring the fluorescence of tryptophan in EF-G, and with GDP, monitoring fluorescence resonance energy transfer (FRET) from Trp to mant-GDP (Wilden et al., 2006) demonstrated that EF-G(H91A) bound GTP and GDP with wild-type affinity (Figure 4-5), confirming that GTP hydrolysis, rather than GTP binding, was abolished by the H91A mutation. 


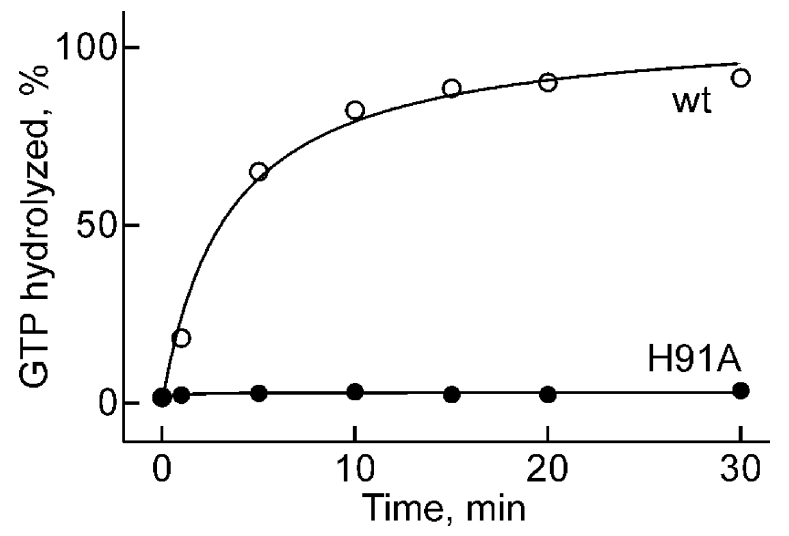

Figure 4-2: Multiple turnover GTP hydrolysis. Time course of turnover GTP hydrolysis.

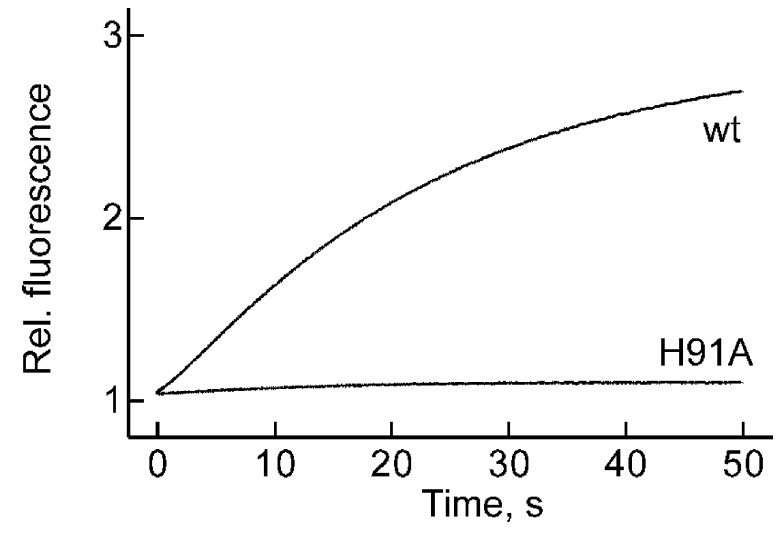

Figure 4-3: Pi release. Turnover GTP hydrolysis monitored by Pi release.

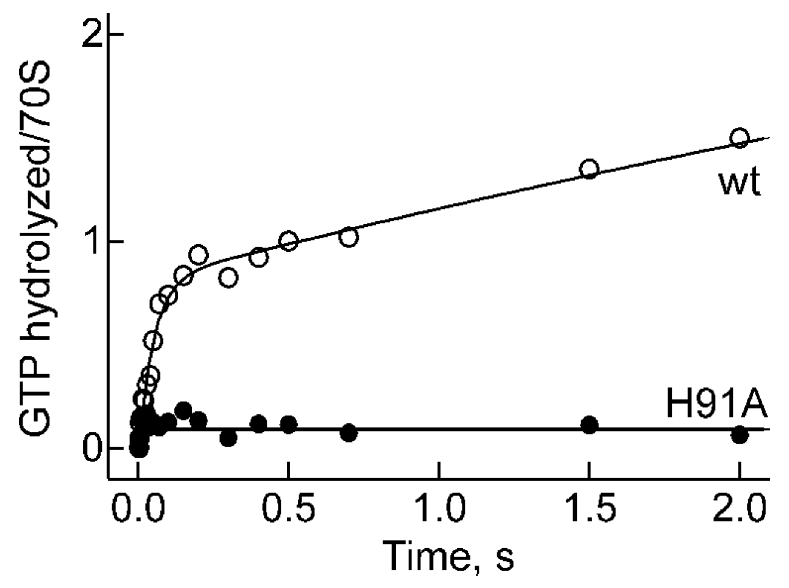

Figure 4-4: Single-round GTP hydrolysis. In the initial burst phase, EF-G hydrolyzed about $0.9 \mathrm{GTP} /$ ribosome. 


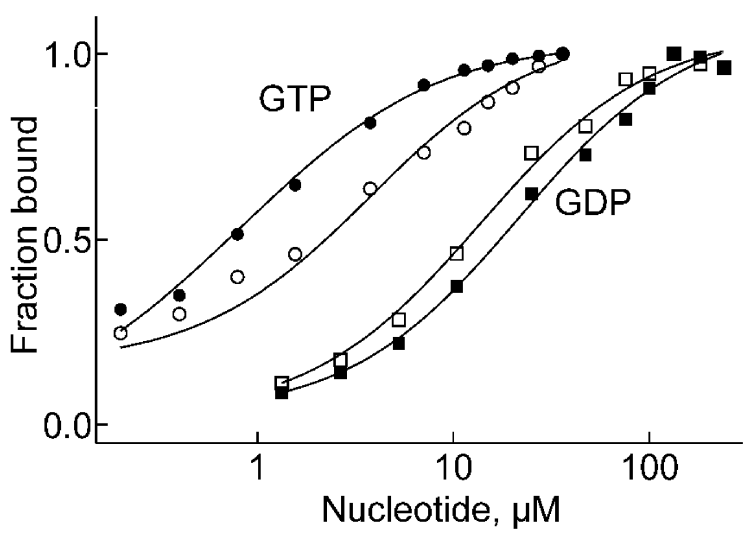

Figure 4-5: Nucleotide binding. Equilibrium titrations of GTP/GDP binding. EF-G (open symbols) or EF-G(H91A) (closed symbols) $(1 \mu \mathrm{M})$ was titrated with increasing amounts of GTP, monitoring Trp fluorescence. GDP titrations were performed with mant-GDP, monitoring mant fluorescence excited by FRET from Trp. $\mathrm{K}_{d}$ values were 1-2 $\mu \mathrm{M}$ for GTP and around $20 \mu \mathrm{M}$ for mant-GDP.

\subsection{Translocation is slow without GTP hydrolysis}

EF-G(H91A) was active in translocation, as shown by the puromycin assay; by contrast to the wt factor, stoichiometric amounts of mutant EF-G to ribosomes were required to bring about translocation on all ribosomes present (Figure 4-6). This indicated that as expected from previous results obtained with non-hydrolyzable analogs of GTP-EF$\mathrm{G}(\mathrm{H} 91 \mathrm{~A})$ catalyzed only a single round of translocation and did not dissociate from the ribosome after one round of translocation.

The kinetics of translocation was studied monitoring the signals from fluorophores in different positions in the pre-translocation complex (Figure 4-7 and Table 3.1). Proflavin attached to the D loop of peptidyl-tRNA (fMetPhe-tRNA ${ }^{\text {Phe }}(\operatorname{Prf})$ ) in the A site reported on tRNA movement from $A$ to $P$ site. The translocation of deacylated tRNA from the $P$ to the $E$ site and its dissociation from the $E$ site was monitored either by FRET from a fluorescein label (Flu), which was attached to 4-thiouracil at position 8 of the tRNA, to a non-fluorescent acceptor attached to position 112 of ribosomal protein S13 (Figure 47) or by the fluorescence change of deacylated tRNA ${ }^{f M e t}(\operatorname{Prf})$ (Pan et al., 2007). The movement of the mRNA on the 30S subunit was monitored by Alexa dyes (Alx488 or Alx405) attached to the 3 ' end (position +14) of the mRNA (Figure 4-7). Rates of steps related to tRNA translocation obtained with Bodipy (Bpy) at the $\mathrm{N}$ terminus of peptidyl- 


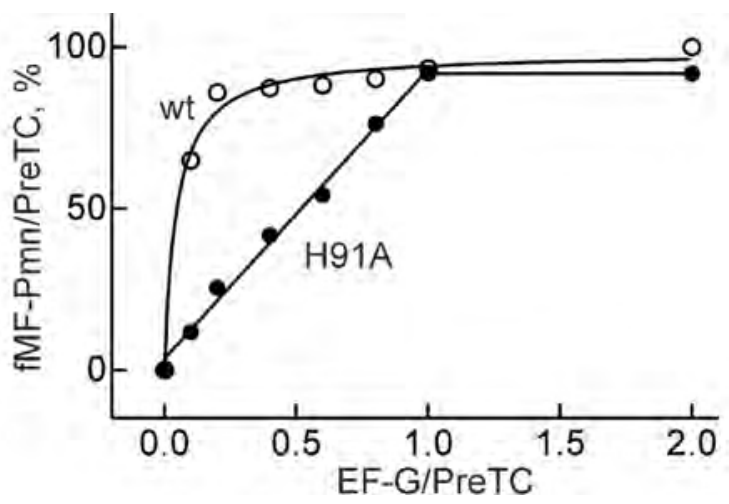

Figure 4-6: Puromycin assay. Translocation monitored by the puromycin reaction. Pre-translocation complexes programed with MF-mRNA were incubated with the indicated amount of EF-G or EF-G(H91A) in the presence of GTP and analyzed by the reaction with puromycin (Pmn). The amount of the product fMetPhe-Pmn (fMF-Pmn) is given relative to the initial amount of fMetPhe-tRNA bound to the ribosome.

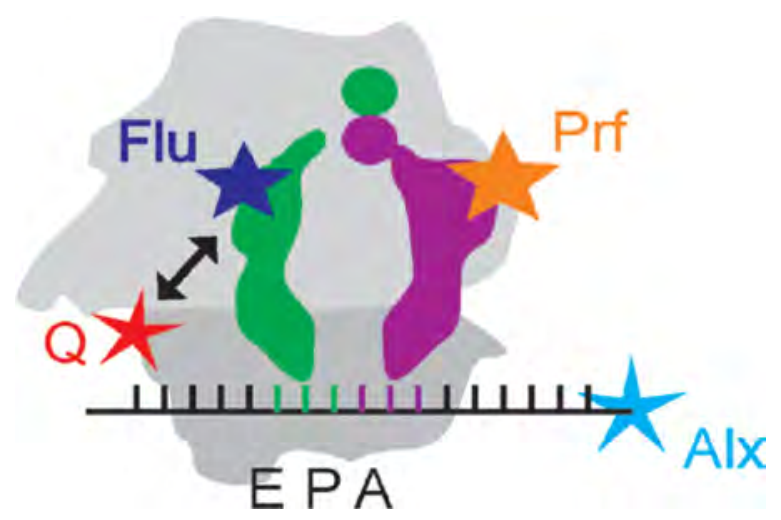

Figure 4-7: Fluorescence labels. Fluorescence labels in the pre-translocation com-

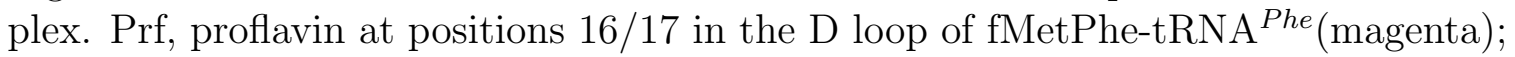
Alx, Alexa488 at position +14 at the 3' end of MF-mRNA, counting from the $\mathrm{A}(+1) \mathrm{UG}$ start codon; Flu, fluorescein at 4-thioU(8) in tRNA ${ }^{f M e t}$ (green); Q, the non-fluorescent acceptor dye Atto540Q at position 112 of protein S13. 


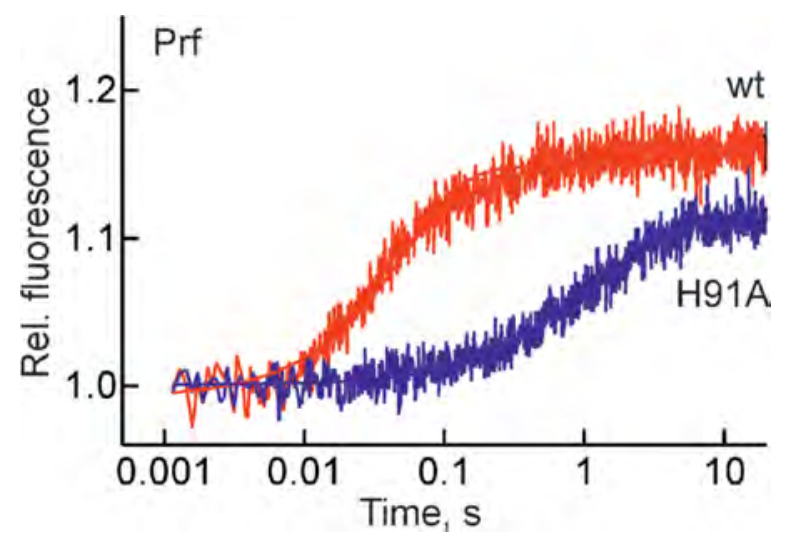

Figure 4-8: Proflavin fluorescence. Time courses of translocation monitored by Prf fluorescence. Stopped-flow traces were evaluated by two-exponential fitting, yielding the values for $\mathrm{k}_{\text {app }}$ summarized in Table 3.1.

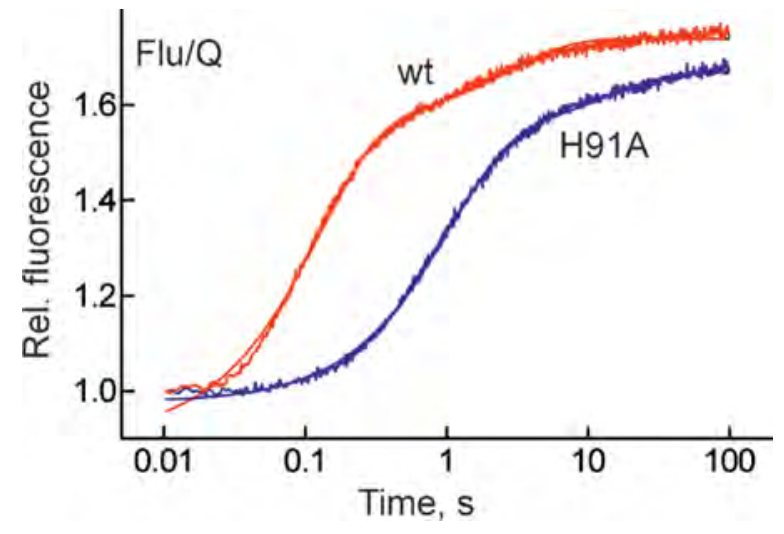

Figure 4-9: Fluorescein fluorescence. Translocation monitored by FRET between Flu in P-site tRNA and Q in protein S13. Stopped-flow traces were evaluated by two-exponential fitting, yielding the values for $\mathrm{k}_{a p p}$ summarized in Table 3.1.

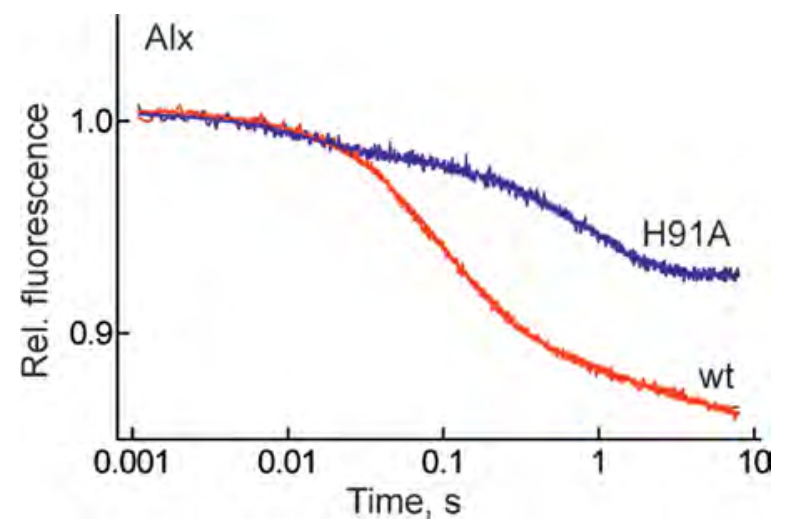

Figure 4-10: Alexa 488 fluorescence. Translocation monitored by Alx488 at the 3' end of the mRNA. Stopped-flow traces were evaluated by two-exponential fitting, yielding the values for $\mathrm{k}_{a p p}$ summarized in Table 3.1. 
Table 4.1: Rate constants of elemental steps of translocation $\left(\mathbf{s}^{-1}\right)$

\begin{tabular}{|c|c|c|c|c|}
\hline Reporter & \multicolumn{2}{|c|}{ EF-G } & \multicolumn{2}{c|}{ EF-G(H91A) } \\
\hline & ${\text { Step } \mathbf{1}^{b}}^{b}$ & Step 2 $^{c}$ & Step 1 $^{b}$ & Step 2 $^{c}$ \\
\hline tRNA (A site) & & & & \\
\hline Bpy & 28 & $-^{d}$ & $9^{e}$ & $0.7^{e}$ \\
\hline Prf16/17 & 30 & & & 1.1 \\
\hline tRNA (P site) & & & & \\
\hline Prf20 & 30 & 13 & n.d. $^{g}$ & n.d. \\
\hline Flu (FRET) & & 10 & & 1 \\
\hline mRNA & & & & \\
\hline Alx405 & 27 & & & 0.8 \\
\hline Alx488 & $35^{h}$ & 6 & $35^{h}$ & $0.9^{e}$ \\
\hline
\end{tabular}

${ }^{a}$ Rate constants were obtained at saturating EF-G concentration $(4 \mu \mathrm{M})$ at $37{ }^{\circ} \mathrm{C} .{ }^{b}$ The step reports on the concomitant movement of peptidyl-tRNA from the A to the $\mathrm{P}$ site, the displacement of deacylated tRNA from the $\mathrm{P}$ site, and the translocation of the mRNA. ${ }^{c}$ The step reports on the final steps of mRNA translocation (Alx488) or the movement of deacylated tRNA through the E site. ${ }^{d}$ The respective step is not reported by the particular fluorescence label. ${ }^{e}$ With EF-G(H91A) the translocation on the 50S subunit is biphasic. The amplitude of the step is about $50 \%$ of that observed with wt EF-G. ${ }^{f}$ The difference between these values and those reported previously (Pan et al., 2007) is due to the temperature difference $\left(37^{\circ} \mathrm{C}\right.$ vs. $\left.25^{\circ} \mathrm{C}\right) .{ }^{g}$ n.d., not determined. ${ }^{h}$ tentative values; the concentration dependence of the respective $\mathrm{k}_{\text {app }}$ suggests a linear dependence; however, the amplitude of the step is too small to allow for precise fitting.

tRNA are also included (Table 3.1). The step assignment and detailed kinetic analysis for the different reporter groups can be found elsewhere (Rodnina et al., 1997, Savelsbergh et al., 2003, Peske et al., 2004, Pan et al., 2007).

Translocation resulted in a major fluorescence change with every label used. The rate of translocation, as reported by $\mathrm{fMetPhe-tRNA}^{\text {Phe }}$ (Prf) (Figure 4-8) or mRNA-Alx405 (Holtkamp et al., 2013) with EF-G(H91A) was $1 \mathrm{~s}^{-1}$, compared with about $30 \mathrm{~s}^{-1}$ with wt EF-G (Table 3.1). The 30-fold rate decrease is similar to previously reported values obtained with non-hydrolyzable GTP analogs, consistent with an important contribution of GTP hydrolysis to translocation (Rodnina et al., 1997, Katunin et al., 2002). The amplitudes of the fluorescence changes of Prf and Alx405 observed with wt and mutant EF-G were very similar (documented in Figure 4-8 for Prf), indicating that despite the 
different rates the reactions went to completion regardless of whether GTP was hydrolyzed (Figure 4-6). In contrast, the label at the 3' end of peptidyl-tRNA(Bpy) reported that translocation on the $50 \mathrm{~S}$ subunit was biphasic with $\mathrm{EF}-\mathrm{G}(\mathrm{H} 91 \mathrm{~A})$, with a fast step that did not require GTP hydrolysis $\left(9 \mathrm{~s}^{-1}\right)$ and a second, slow step that was dependent on $30 \mathrm{~S}$ translocation $\left(1 \mathrm{~s}^{-1}\right.$ ) (Table 3.1, Holtkamp et al., 2013, and further discussed in the next sections). The apparent rate of deacylated tRNA release from the $E$ site in the presence of wt EF-G was about $10 \mathrm{~s}^{-1}$, based on both tRNA ${ }^{f M e t}$ (Prf) fluorescence and S13-tRNA ${ }^{f M e t}(F l u)$ FRET assays (Figure 4-9, Table 3.1). Notably, the rate of $A$ to $\mathrm{P}$ site translocation on ribosomes carrying labels in S13 and tRNA ${ }^{f M e t}(\mathrm{Flu})$ monitored by the reporter in mRNA-Alx405 was the same as on unmodified ribosomes $\left(30 \mathrm{~s}^{-1}\right)$, indicating that the labels per se did not affect the kinetics of translocation. The $10 \mathrm{~s}^{-1}$ rate represents a global rate of tRNA displacement from the $P$ to the $E$ site followed by the dissociation of the E-site tRNA from the ribosome, because at our concentrations and buffer conditions (low $\mathrm{Mg}^{2+}$ concentrations and no polyamines) essentially all deacylated tRNA dissociates from the ribosome following translocation to the $E$ site. Deconvoluting the rates of the individual reactions yielded an elemental rate constant of E-site clearance of about $15 \mathrm{~s}^{-1}$. With EF-G(H91A) the amplitude of the FRET change was the same as with wt EF-G (Figure 4-9), suggesting that tRNA release from the E site was not blocked when GTP was not hydrolyzed. The release rate $\left(1 \mathrm{~s}^{-1}\right)$ was limited by the preceding tRNA translocation step, which was slow with the mutant factor. Essentially the same kinetics of $\mathrm{P}$ to E-site translocation was observed with tRNA ${ }^{f M e t}(\mathrm{Prf})$ (Table 3.1).

Unexpectedly, translocation monitored by the Alx488 label at the 3' end of the mRNA reported a step that did not follow the pattern of tRNA-mRNA translocation observed with the other labels (Figure 4-10). With wt EF-G, the rate of the dominant step reported by Alx488 was significantly slower $\left(6 \mathrm{~s}^{-1}\right)$ than the rates of translocation on either $30 \mathrm{~S}$ or $50 \mathrm{~S}$ subunits reported by the other labels $\left(30 \mathrm{~s}^{-1}\right)$ (Table 3.1). With EF-G(H91A), the reaction was as slow as translocation $\left(1 \mathrm{~s}^{-1}\right)$ (Table 3.1); however, the fluorescence change did not reach the full amplitude (Figure 4-10). This may suggest that Alx488 at the $3^{\prime}$ end of the mRNA reports on a step in translocation which is physically distinct from mRNA-tRNA displacement itself and is affected by GTP hydrolysis. Notably, the 
reduced amplitude was not due to EF-G remaining bound after translocation, because the full fluorescence amplitude due to mRNA-Alx488 translocation was observed in the presence of fusidic acid (FA) (data not shown), which freezes EF-G on the ribosome in a post-translocation state (Savelsbergh et al., 2009).

\subsection{Direct observation of $30 \mathrm{~S}$ and $50 \mathrm{~S}$ transloca- tion}

Movement on the $30 \mathrm{~S}$ subunit has been observed using different environmentally-sensitive fluorescence reporters, such as pyrene and fluorescein attached to the 3 ' end of the mRNA (Studer et al., 2003, Peske et al., 2004, Dorner et al., 2006; Ermolenko and Noller, 2011); however, due to the lack of suitable reporters, the tRNA movement on the $50 \mathrm{~S}$ subunit has not been examined kinetically. To study tRNA translocation on the $50 \mathrm{~S}$ subunit, we utilized BODIPY-FI (Bpy) attached to the N-terminal methionine of the nascent peptide. Bpy does not interfere with translation and appears to be an ideal fluorophore due to small size and advantageous, well-characterized spectroscopic properties (Ellis et al., 2008). The translocation of tRNA and mRNA on the $30 \mathrm{~S}$ and $50 \mathrm{~S}$ subunits of Escherichia coli ribosomes was monitored using pre-translocation complexes with reporter groups attached to both the N-terminal methionine of the nascent peptide (Bpy) and the 3' end of the mRNA (Alexa405) (Figure 4-11). The latter fluorophore was chosen because its fluorescence can be monitored selectively also in the presence of Bpy. When translocation on double-labeled pre-translocation complexes with mRNA-Alx, deacylated tRNA ${ }^{f M e t}$ in the $P$ site and Bpy-MetPhe-tRNA ${ }^{P h e}$ in the $A$ site was initiated by rapid mixing with EF-G and GTP in a stopped-flow apparatus, we observed a fluorescence decrease of the two reporters measured in parallel. Comparison of the rates of $50 \mathrm{~S}$ and $30 \mathrm{~S}$ translocation showed that the movements on the two subunits took place at the same rate, about 28 $\mathrm{s}^{-1}$ at saturation with EF-G (Figure 4-11 and Table 3.2), indicating simultaneous movement on the two subunits. A translocation rate of about $25 \mathrm{~s}^{-1}$ was also observed when fMetPhe-tRNA $^{\text {Phe }}$ movement was monitored by a proflavin label in the elbow region of 


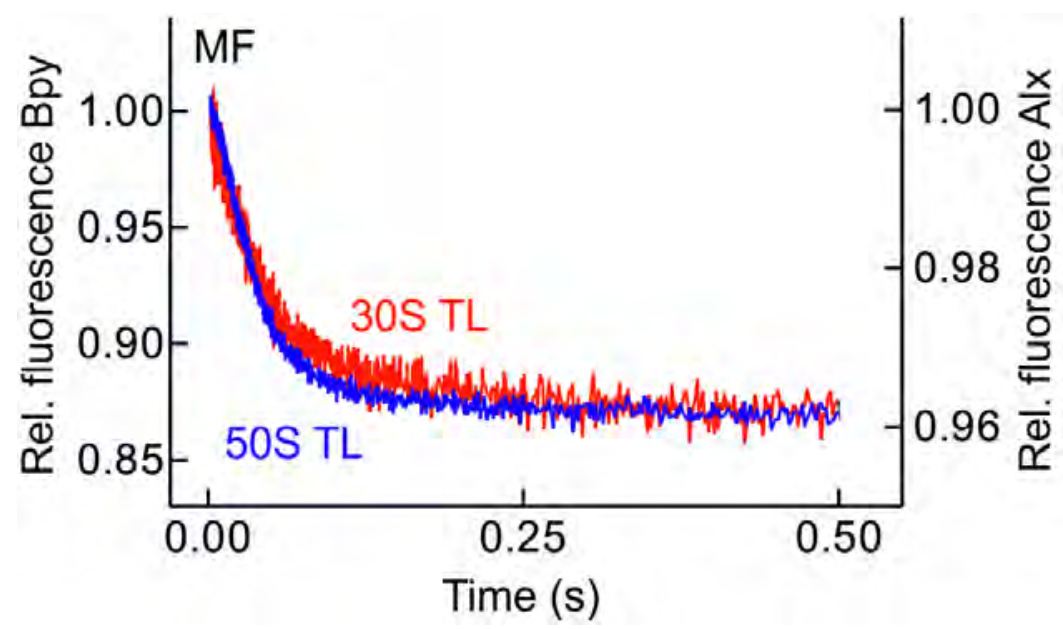

Figure 4-11: Kinetics of $50 \mathrm{~S}$ and $30 \mathrm{~S}$ translocation. Time courses of BpyMetPhe-tRNA ${ }^{\text {Phe }}$ translocation on the $50 \mathrm{~S}$ subunit (50S TL; blue trace) and mRNAAlx translocation on the $30 \mathrm{~S}$ subunit (30S TL; red trace) after rapid mixing of EF-G (4 $\mu \mathrm{M}$, final concentration after mixing) with the double-labeled pre-translocation complex. Figure courtesy of Wolf Holtkamp.

the tRNA (Table 3.2), a well-characterized label previously used to study the kinetics of translocation (Rodnina et al., 1997, Savelsbergh et al., 2003, Pan et al., 2007). 50S and $30 \mathrm{~S}$ translocation were also synchronous at $23^{\circ} \mathrm{C}$, albeit slower than at $37^{\circ} \mathrm{C}$, about 6 $\mathrm{s}^{-1}$, in agreement with values reported by the proflavin label (Pan et al., 2007). Thus, EF-G-GTP binding and GTP hydrolysis promote the synchronous forward movement of both acceptor and anticodon domains of the tRNAs together with the mRNA, and the elbow region of peptidyl-tRNA on the two ribosomal subunits.

To further assign the molecular basis for the Bpy fluorescence change, we next asked the question of whether or not it is sensitive to the formation of hybrid states. At our buffer conditions (low $\mathrm{Mg}^{2+}$, no polyamines, $37^{\circ} \mathrm{C}$ ) we expect the majority of the ribosomes in the pre-translocation state to be in the hybrid, rotated state (Kim et al., 2007). If the Bpy reporter were sensitive to the classic-to-hybrid transition, then we would expect a change in the amplitude of the fluorescence signal (and potentially changes in the observed rates) when translocation starts on the pre-translocation complexes that are in the classic state. Preferential stabilization of the classical state can be induced by increasing $\mathrm{Mg}^{2+}$ concentration (Kim et al., 2007). We measured the changes of Bpy fluorescence in the range of $\mathrm{Mg}^{2+}$ concentrations between $3.5 \mathrm{mM}$ and $20 \mathrm{mM}$. The rate was reduced 2-fold 
Table 4.2: Rate constants of $50 \mathrm{~S}$ and $30 \mathrm{~S}$ translocation.

\begin{tabular}{|c|c|c|c|}
\hline EF-G/antibiotic & \multicolumn{2}{|c|}{ 50S translocation } & \\
\hline & $\mathbf{k}_{50 \text { Sfast }}$ & $\mathbf{k}_{\text {50Sslow }}$ & $\mathbf{k}_{30 S}$ \\
\hline wt $^{b} /$ no antibiotic & $28 \pm 3^{c}$ & $-d$ & $27 \pm 2^{e}$ \\
\hline EF-G(H91A) & $9 \pm 1$ & $0.7 \pm 0.1$ & $0.9 \pm 0.2$ \\
\hline EF-G(H583K) & $25 \pm 1$ & $0.7 \pm 0.1$ & $1.0 \pm 0.2$ \\
\hline EF-G $(\Delta 4 / 5)$ & & $0.06 \pm 0.01$ & $0.04 \pm 0.02$ \\
\hline
\end{tabular}

${ }^{a}$ Rate constants were obtained at saturating EF-G concentration $(4 \mu \mathrm{M})$ at $37{ }^{\circ} \mathrm{C} .{ }^{c}$ Data from Holtkamp et al., 2013. ${ }^{b}$ wt, wild type. ${ }^{d}$-, not observed, i.e., the amplitude of the signal change is less than $10 \%$ of the total amplitude observed with wt EF.G in the absence of antibiotics. ${ }^{e}$ The reported rates for $\mathrm{k}_{30 S}$ pertain to the steps that constitute $80 \%$ or more of the total reaction amplitudes.

at saturating high $\mathrm{Mg}^{2+}$, suggesting that EF-G-dependent $50 \mathrm{~S}$ translocation is somewhat slower when the majority of ribosomes start from the classical, rather than the hybrid state. However, the amplitude of the signal did not change, suggesting that Bpy is not sensitive to the classical/hybrid state transition and reflects the transition from either $A / A$ or $\mathrm{A} / \mathrm{P}$ to the $\mathrm{P} / \mathrm{P}$ state.

\subsection{Role of GTP hydrolysis}

GTP hydrolysis strongly increases the overall rate of translocation (results above, Rodnina et al., 1997, Katunin et al., 2002, Pan et al., 2007). To assess whether or not the effect is subunit-specific, we used EF-G(H91A), a GTPase-deficient EF-G mutant. When pretranslocation complexes were mixed with $\mathrm{EF}-\mathrm{G}(\mathrm{H} 91 \mathrm{~A})$, the fluorescence of Bpy and $\mathrm{Alx}$ decreased to similar levels as with wild-type (wt) EF-G (Figure 4-12); however, the rates were lowered substantially (Table 3.2). The rate of $30 \mathrm{~S}$ translocation was reduced to about $1 \mathrm{~s}^{-1}, 30$-fold lower than with wt EF-G. The kinetics of $50 \mathrm{~S}$ translocation became biphasic (Figure 4-12A), with a fast step $\left(11 \mathrm{~s}^{-1}\right.$ at saturation with EF-G(H91A) (Figure 4-13A). That was only three times slower than with wt EF-G and an additional slow step which contributed about $50 \%$ of the amplitude and had a rate of $1 \mathrm{~s}^{-1}$ at EF-G saturation. The latter rate coincided with the rate of $30 \mathrm{~S}$ translocation (Figure 4-13B). Thus, when GTP 
A

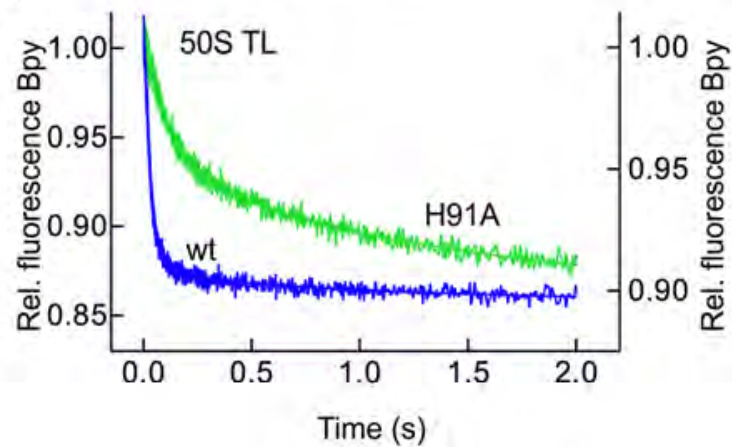

B

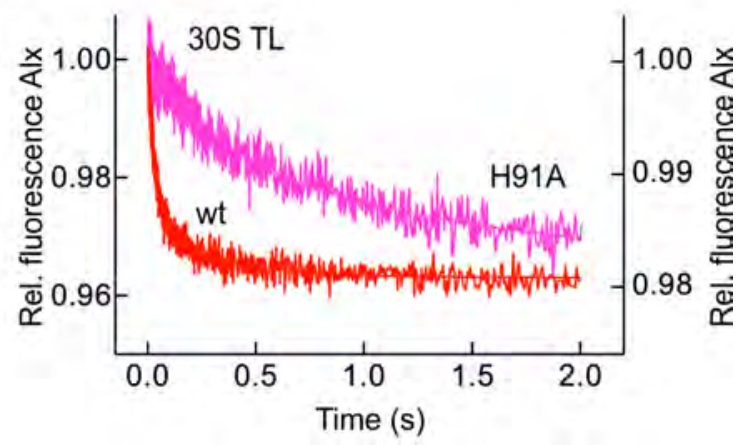

Figure 4-12: Inhibition of translocation by mutations in EF-G(A) Time courses of 50S translocation in the presence of GTPase-deficient EF-G(H91A) (green, right Y-axis) compared to wt EF-G (wt, blue, left Y-axis). (B) Time courses of 30S translocation in the presence of the GTPase-deficient EF-G(H91A) (pink, right Y-axis) compared to wt EF-G (wt, red, left Y-axis).

hydrolysis was blocked, the two steps of $50 \mathrm{~S}$ translocation were uncoupled. The formation of the intermediate $50 \mathrm{~S}$ translocation state required EF-G binding, but not GTP hydrolysis, whereas the transition from the intermediate to the $50 \mathrm{~S}$ post-translocation state was linked to 30 S translocation, which, in turn, was coupled to GTP hydrolysis.

Domain 4 of EF-G is known to play an important role in translocation by coupling the conformational rearrangements of EF-G to forward movement of the tRNAs (Rodnina et al., 1997, Peske et al., 2000, Savelsbergh et al., 2000, Peske et al., 2004). When translocation experiments were performed with mutant EF-G lacking domains 4 and 5 (EF$\mathrm{G}(\Delta 4 / 5)$ ), both $50 \mathrm{~S}$ and $30 \mathrm{~S}$ translocation were severely inhibited (Table 3.2). Because EF-G $(\Delta 4 / 5)$ binds to the ribosome and hydrolyzes GTP as rapidly as wt EF-G (Savelsbergh et al., 2000), translocation with EF-G $(\Delta 4 / 5)$ must be inhibited directly after the GTP hydrolysis step, presumably by an inhibition of the rate-limiting rearrangement of the ribosome that controls tRNA translocation and Pi release (Savelsbergh et al., 2003). The mutation of the conserved His583 at the tip of domain 4 to Lys (EF-G(H583K)) does not interfere with GTP hydrolysis, Pi release, or the dissociation of EF-G from the ribosome (Savelsbergh et al., 2000). Similar to the effect of the H91A mutation, 50 S translocation catalyzed by EF-G(H583K) was biphasic (Table 3.2). The rapid step was independent of $30 \mathrm{~S}$ translocation and led to an intermediate that was not puromycin-reactive. The 
A

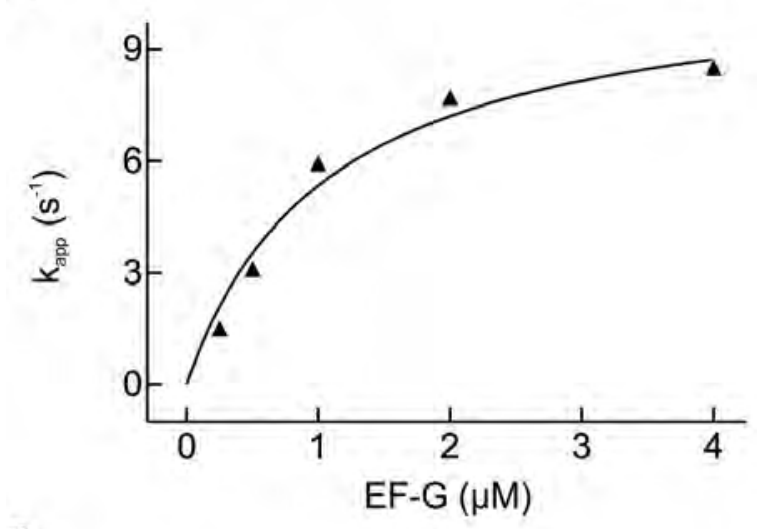

B

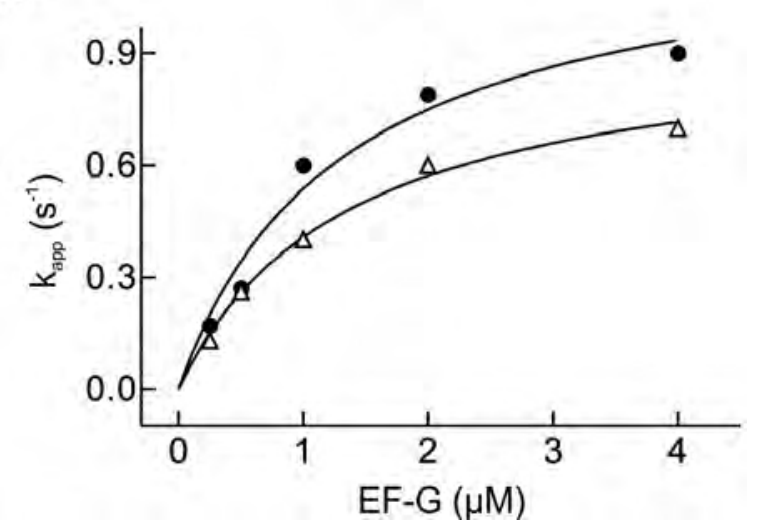

Figure 4-13: Concentration dependence of apparent rate constants of translocation promoted by EF-G(H91A).(A) The rate of the fast step of 50S translocation (filled triangle). (B) The rates of the slow step of $50 \mathrm{~S}$ translocation (empty triangle) and 30S translocation (filled circle). 

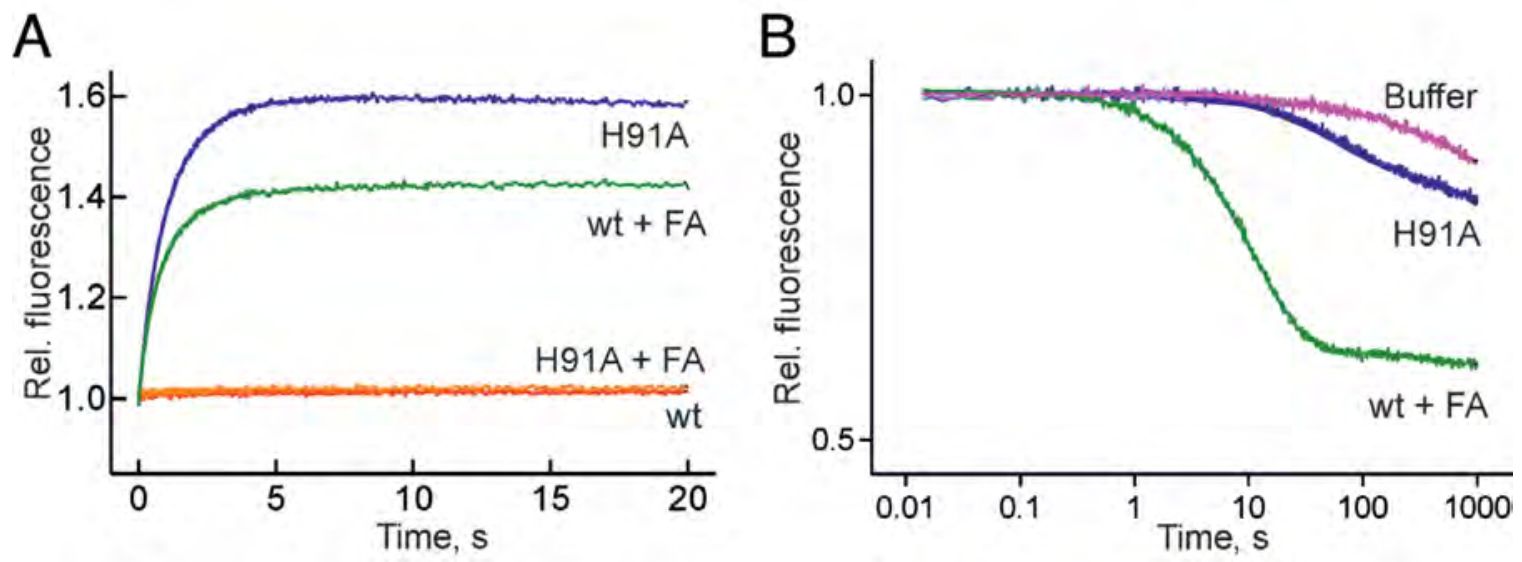

Figure 4-14: EF-G stability on the ribosome. (A) Time courses monitored by mant-nucleotide fluorescence. Red trace, wt EF-G + mant-GTP; green trace, wt EFG with mant-GDP and FA (0.2 mM); blue trace, EF-G(H91A) + mant-GTP; orange trace, EF-G(H91A) + mant-GDP with FA. (B) Stability of ribosome-EF-G-mantGTP complexes. The complex with wt EF-G + FA was chased with excess GDP (green trace), the complex with EF-G(H91A) with excess GDP (blue trace).

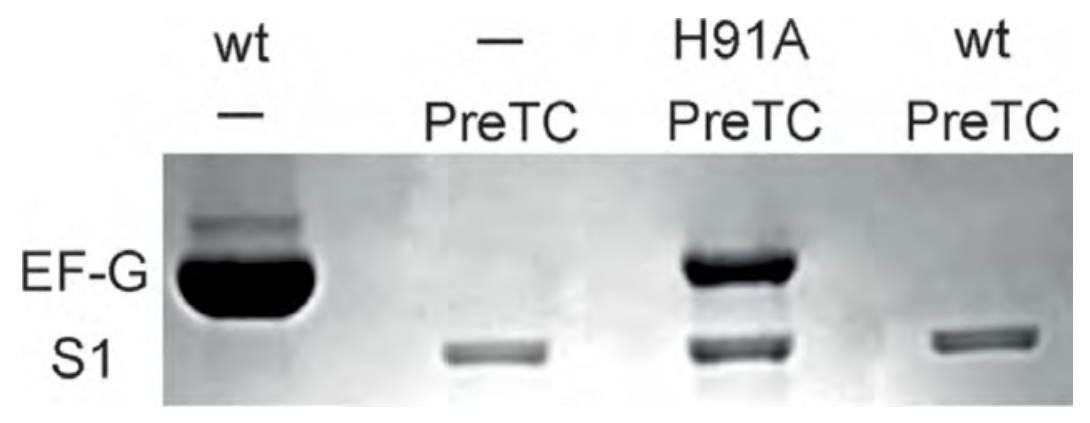

Figure 4-15: EF-G binding to pre-translocation complex. EF-G and ribosomal protein S1 were visualized by Coomassie staining. PreTC, pre-translocation complex

slower step proceeded at the rate of $30 \mathrm{~S}$ translocation and led to the post-translocation state (Table 3.2). The rate of $30 \mathrm{~S}$ translocation was reduced substantially, suggesting a specific effect of domain 4 on mRNA-tRNA movement on the $30 \mathrm{~S}$ subunit.

\subsection{The complex of ribosomes with EF-G(H91A)- GTP is very stable}

With wt EF-G, the initially formed ribosome-EF-G-GTP complex, which is unstable and dissociates rapidly $\left(\mathrm{k}_{o f f} \approx 100 \mathrm{~s}^{-1}\right.$ ) (Katunin et al., 2002), rearranges to form the GTPase- 


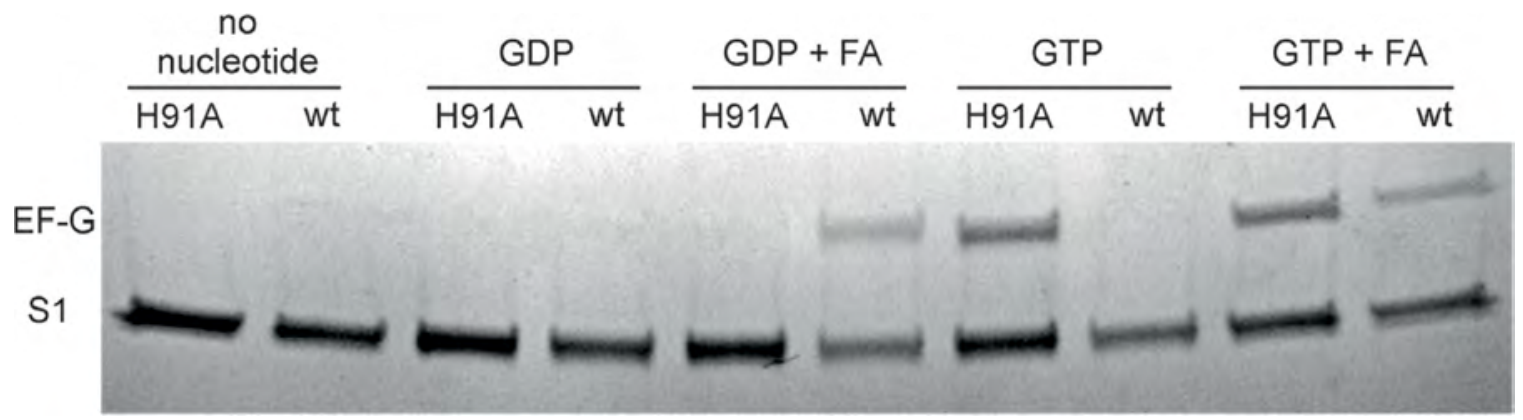

Figure 4-16: EF-G binding to the ribosome. Conditions as in Figure 4-15 but with $5 \mu \mathrm{M}$ mant-nucleotides and $0.2 \mathrm{mM}$ FA for comparison with Figure 4-14.

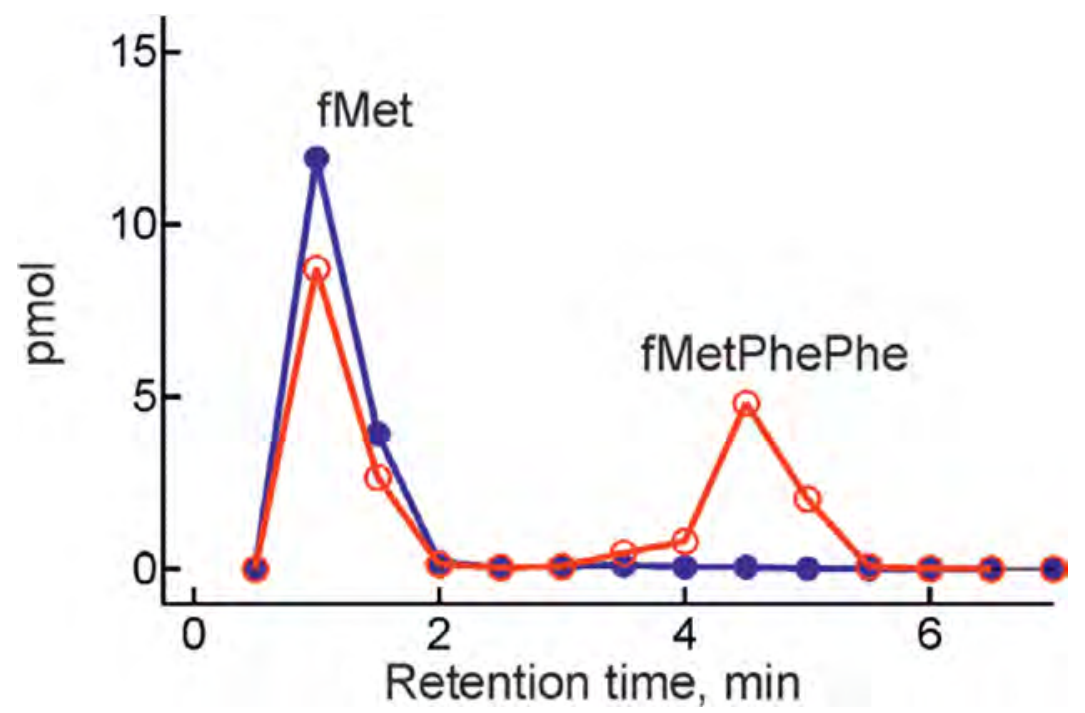

Figure 4-17: EF-G and EF-Tu competing binding site. Peptide formation. Initiation complexes programed with MFF-mRNA and containing fMet-tRNA ${ }^{\text {fMet }}$ in the $\mathrm{P}$ site were pre-incubated with wt EF-G (red) or EF-G(H91A) (blue) in the presence of GTP and mixed with EF-Tu-GTP-Phe-tRNA ${ }^{\text {Phe }}$. fMetPhePhe tripeptide was separated from unreacted fMet by HPLC. 
activated complex, which subsequently hydrolyzes GTP (Rodnina et al., 1997). The resulting ribosome-EF-G-GDP-Pi complex does not accumulate, as monitored by the fluorescence of mant-GTP that was excited via FRET from a tryptophan residue in EF-G (Figure 4-14A), likely because it is consumed in the following steps that lead to factor dissociation. By contrast, when GTP hydrolysis is blocked, as in EF-G(H91A)-GTP, the increase of mant-GTP fluorescence reports the formation of a ribosome-EF-G-mant-GTP complex at a rate of around $1 \mathrm{~s}^{-1}$ (Figure 4-14A), whereas with mant-GDP the stable complex was not formed, even though EF-G(H91A) could bind mant-GDP (Figure 4-5). When the dissociation of wt EF-G from the ribosome was blocked by FA the high-fluorescence intermediate was stabilized, although the amplitude of fluorescence change was smaller than with EF-G(H91A) (Figure 4-14A). The smaller amplitude may either be due to the fact that FA is not very effective as an inhibitor (Seo et al., 2006, Savelsbergh et al., 2009) allowing partial dissociation of EF-G-mant-GDP from the ribosome, or indicate that the complexes with EF-G(H91A)-GTP and EF-G-GDP-FA are structurally different. To examine that possibility, we tested the stability of mant-GTP/mant-GDP binding to EF-G on the ribosome by competition with excess unlabeled GTP or GDP; the observed rates represent the dissociation rate constant of mant-GTP/mant-GDP from EF-G bound to the ribosome. The complex was almost 10 times more stable with $\mathrm{EF}-\mathrm{G}(\mathrm{H} 91 \mathrm{~A})$ than with EF-G-FA (Figure 4-14B), which suggests that the ribosome-EF-G complex formed with GTP is structurally different from that formed after GTP hydrolysis and Pi release in the presence of FA.

The high stability of ribosome-EF-G(H91A)-GTP complex could also be demonstrated by pull-down experiments. The complex remained intact during centrifugation through a sucrose cushion, in contrast to the complex of ribosomes with wt EF-G, which was not observed (Figure 4-15). Retention of EF-G(H91A) to the ribosome required GTP, suggesting that it is specifically the GTP-bound conformation of the factor that remained stably bound (Figure 4-16). In contrast, the stable binding of wt EF-G to the ribosome required addition of FA and was observed with both GDP and GTP (Figure 4-16); the latter is hydrolyzed to GDP within the time of experiment.

In keeping with these results, in the complex of EF-G(H91A)-GTP with ribosomes the 
mutant factor was bound strongly enough to prevent the binding of the ternary complex EF-Tu-GTP-Phe-tRNA ${ }^{\text {Phe }}$. This is evident from the lack of $\mathrm{fMetPhe}$ and $\mathrm{fMetPhePhe}$ diand tripeptide formation on ribosomes programed with MFF-mRNA when EF-G(H91A) was preincubated with the ribosome prior to the addition of the EF-Tu complex (Figure 417). Thus, following (slow) translocation, EF-G in the GTP-bound form is stalled on the ribosome in a conformation that differs from that induced by FA. 


\section{5}

\section{Discussion}

\subsection{EF-G works in a dual energy regime ${ }^{1}$}

EF-G hydrolyzes GTP very rapidly, much faster than the displacement of mRNA and tRNA takes place (Rodnina et al., 1997, Pan et al., 2007), which makes it difficult to deconvolute the potential effects of GTP hydrolysis on the elemental steps of translocation. The use of a GTPase-deficient mutant of EF-G allows the dissection of the EF-G cycle into GTPasedependent and independent steps, using the authentic GTP-bound form of the factor, thereby providing an insight into the energy regime of translocation. The advantage of the H91A mutation is that it does not change the affinity of GTP or GDP binding, indicating that the mutation does not affect the structure and dynamics of the nucleotide binding pocket. Due to the lack of GTP hydrolysis, the conformational switch from the GTP- to the GDP-bound form of EF-G is blocked, and the GTP-bound conformation of the factor is favored; therefore, GTP can be used rather than GTP analogs which may not mimic GTP perfectly.

By analogy to EF-Tu, where the mutation of the catalytic histidine reduces the rate of GTP hydrolysis by five orders of magnitude (Daviter et al., 2003), the H91A mutation in EF-G inhibits GTP hydrolysis, in this case virtually completely, as there is no measurable GTPase activity of the factor, even when it is bound to the ribosome. The complete

\footnotetext{
${ }^{1}$ CUNHA, C. E., BELARDinelli, R., PESKe, F., HOLTKAMP, W., WINTERMEYER, W. \& RODNINA, M. V. 2013. Dual use of GTP hydrolysis by elongation factor G on the ribosome. Translation, 1, e24315.
} 
loss of the GTPase activity of EF-G(H91A) suggests that the detailed mechanism of GTP hydrolysis, and the contributions of different groups to catalysis, may differ in EF$\mathrm{G}$ and EF-Tu, despite the high sequence conservation in their GTP-binding domains. Consistent with this notion, the intrinsic GTPase activities of EF-Tu and EF-G also differ, ranging from the low, but measurable activity of EF-Tu $\left(10^{-5} \mathrm{~s}^{-1}\right)$ to practically no activity of EF-G. Biochemical evidence suggests that without GTP hydrolysis EF-G brings about one round of translocation, but is not capable of turnover; therefore, to promote translocation on a given amount of pre-translocation complexes, stoichiometric amounts of $E F-G(H 91 A)$ are required, rather than the catalytic amounts of wt EF-G that suffice to promote translocation on excess ribosomes in a turnover reaction.

The use of fluorophores at different positions in the pre-translocation complex revealed not only movements of the tRNAs and the mRNA from $A$ to $P$ and $P$ to $E$ sites, but also reported on tRNA dissociation from the ribosome and on rearrangements within the $30 S$ subunit which have not been observed before. Thus, the present work provides a comprehensive velocity map of translocation with and without GTP hydrolysis. Several labels robustly report translocation of mRNA and tRNAs from $A$ to $P$ and $P$ to $E$ sites, including $30 \mathrm{~S}$ translocation monitored by mRNA-Alx405, $50 \mathrm{~S}$ translocation of tRNA by Bpy, and the movement of the tRNA elbow regions by Prf labels in peptidyl-tRNA and deacylated tRNA. For translocation catalyzed by wt EF-G with GTP, all these labels reported a rate of about $30 \mathrm{~s}^{-1}$ (Table 3.1). The Prf label in tRNA fMet additionally reported on the movement of deacylated tRNA out of the E site (Pan et al., 2007), taking place at a rate of $10-13 \mathrm{~s}^{-1}$. A similar rate was observed when FRET between deacylated tRNA and the ribosome, labeled at protein S13, was monitored. This lower rate can be explained by the step-wise nature of the dissociation of the P-site tRNA, which has to move from the $P$ to the $E$ site (likely with the same rate as the $A$ to $P$ site movement of peptidyl-tRNA) before it dissociates from the $E$ site into solution; this requires additional time and thus lowers the overall rate of the process. In translocation promoted by EF-G(H91A)-GTP all steps proceed at a uniform rate of $1 \mathrm{~s}^{-1}$, suggesting that the lack of GTP hydrolysis impairs a step that precedes, or takes place concomitantly with, tRNA movement and is rate-limiting for the following steps. The only exception 
is the Bpy label on the 3' end of peptidyl-tRNA on the $50 \mathrm{~S}$ subunit which apparently proceeds in two steps, reaching an intermediate position independently of GTP hydrolysis and then moving to the post-translocation state at the rate of slow $30 \mathrm{~S}$ translocation. In contrast to previous results reported for translocation with EF-G and GDPNP (Spiegel et al., 2007), in translocation promoted by EF-G(H91A)-GTP the deacylated tRNA did not accumulate in the $\mathrm{E}$ site, suggesting that GTP hydrolysis is not required for E-site clearance and indicating that, at least for this step, GDPNP is not a perfect mimic of GTP. Thus, part of the energy of GTP hydrolysis is used to drive a conformational change of the ribosome that controls translocation; the following tRNA release itself is spontaneous.

One unexpected result concerns the differences in steps reported by the two fluorescence labels in the mRNA, Alx405 and Alx488. Biochemically, the complexes bearing the two mRNAs are very similar, i.e., in both cases the label is attached at position +14 and the extent of complex formation and translocation is the same. However, mRNA-Alx405 reports a step that kinetically coincides with the rate of tRNA movement both on the 50S subunit and at the elbow region $\left(30 \mathrm{~s}^{-1}\right)$, whereas the major step reported by mRNAAlx488 is significantly slower $\left(6 \mathrm{~s}^{-1}\right)$. We hypothesize that on the ribosome the two labels are oriented differently, with Alx405 facing the 30S body and therefore reporting on the mRNA displacement relative to the body of the $50 \mathrm{~S}$ subunit, and Alx488 following the movements of the $30 \mathrm{~S}$ head, which are only loosely coupled to the body movements (Fischer et al., 2010, Guo et al., 2012). If our interpretation is correct, then the rate of backward head movement is the slowest step of EF-G-dependent translocation and may fully or partially determine the rate of EF-G release and EF-G turnover $\left(2-5 \mathrm{~s}^{-1}\right.$ at our conditions (Mohr et al., 2002, Savelsbergh et al., 2005)). A similar rate for the backward head movement, $10 \mathrm{~s}^{-1}$, has been observed by FRET measurements (Guo et al., 2012); however, given the difference in buffer conditions, the comparison should be made with caution. With EF-G(H91A), this step is as slow as all other steps of translocation, $1 \mathrm{~s}^{-1}$, but occurs only partially, as evident from the 50\% smaller amplitude, compared with wt EF-G. This suggests that in the absence of GTP hydrolysis the $30 \mathrm{~S}$ head may be trapped in a distinct intermediate state between the pre- and post-translocation positions or may reversibly fluctuate between those states. Thus, GTP hydrolysis is not only important for 
the acceleration of translocation, but may also be essential for the backward rotation of the 305 head.

EF-G(H91A)-GTP forms a very tight complex with the ribosome which may resemble the structure of an intermediate complex with EF-G-GDP-FA revealed by cryo-EM reconstruction (Ratje et al., 2010). In that work, the ribosome complexes contained deacylated tRNA in the $P$ site, and EF-G-GDP was stabilized in the $A$ site by FA. By computational sorting the authors identified two complexes, one with the tRNA in the P/E state, which they denoted as pre-translocation intermediate $\left(\mathrm{TI}^{P R E}\right.$; we note, however, that the $\mathrm{A}$ site of the ribosome contained no tRNA), and the second with the P-site tRNA in intermediate pe/E position, denoted $\mathrm{TI}^{P O S T}$. In the latter complex, EF-G with domain 4 reached into the $30 \mathrm{~S}$ A site, and peptidyl-tRNA could be modeled in an ap/P position without clashing with EF-G (Ratje et al., 2010). In the pe/E position, the tRNA maintained contact with the $\mathrm{P}$ site on the $30 \mathrm{~S}$ head and simultaneously established an interaction with the $\mathrm{E}$ site on the $30 \mathrm{~S}$ platform; domain 4 of EF-G interacted with the $30 \mathrm{~S}$ head domain, stabilizing the swiveled conformation. While the elbow region and the 3 ' end of the tRNA were essentially in their post-translocation position, the translocation of the mRNA with the tRNA anticodons is incomplete with respect to the $30 \mathrm{~S}$ head. If $E F-G(H 91 A)$ stabilized the same or a similar state, one would expect that labels at the 3' end of the mRNA are differently affected by mRNA translocation, depending on their distance to the $30 \mathrm{~S}$ head and platform, as we hypothesize for mRNA-Alx488 and mRNA-Alx405. This assignment of steps would support the notion that the pe/E state is an authentic translocation intermediate and provide a time line to the cryo-EM results. In contrast to the original assignment of the pe/E state (Ratje et al., 2010), our data indicate that the intermediate referred to as $\mathrm{TI}^{P O S T}$ is rather a late pre-translocation intermediate. In comparison, $\mathrm{TI}^{P R E}$ (which equally well can be described as a post-translocation intermediate, because there is no A-site tRNA), might be closer to the intermediate stabilized by FA, in which the translocation is complete on both $50 \mathrm{~S}$ and $30 \mathrm{~S}$ subunit (Agrawal et al., 1998, Stark et al., 2000, Gao et al., 2009, Ratje et al., 2010), consistent with the complex assignment as a post-translocation complex stalled prior to EF-G release.

In agreement with earlier results, our data indicate that GTP hydrolysis is important 
for the release of EF-G from the ribosome. The ribosome-EF-G(H91A)-GTP complex is extremely stable and blocks the access of other factors. In the GTP-bound form of EF-G, the stability of nucleotide binding in the complex is very high, consistent with the low nucleotide exchange rates observed with GDPNP (Wilden et al., 2006). In comparison, nucleotide exchange in the FA-stalled ribosome-EF-G complex is almost 10 times faster, suggesting that the structure of the nucleotide binding pocket of EF-G is relaxed upon GTP hydrolysis and Pi release, independent of the presence of FA (Savelsbergh et al., 2009).

The acceleration of translocation by GTP hydrolysis indicates that in the early phase of translocation EF-G functions as a motor protein that uses the free energy of GTP hydrolysis to drive the movement. Such a function implies that the transition from EFG-GTP to EF-G-GDP-Pi is accompanied by a structural rearrangement of the factor that is coupled to a conformational change of the pre-translocation complex (Savelsbergh et al., 2003). The energy of GTP hydrolysis is used to accelerate the movement of both tRNAs relative to the $50 \mathrm{~S}$ subunit and the body of the $30 \mathrm{~S}$ subunit. The completion of the reaction on the $30 \mathrm{~S}$ subunit, likely by the backward swivel of the $30 \mathrm{~S}$ subunit and the dissociation of EF-G, requires another structural change which presumably is driven by the release of $\mathrm{Pi}$ (Savelsbergh et al., 2009). The conformational switch is inhibited, and, with that, the dissociation of EF-G from the ribosome, when either the GTPase-inactive EF-G mutant or non-hydrolyzable GTP analogs are used or when the nucleotide binding site retains $\mathrm{Pi}$ (Savelsbergh et al., 2005) or vanadate mimicking Pi (Savelsbergh et al., 2009). Thus, there are two energy regimes related to different phases of translocation by which EF-G uses GTP hydrolysis. In the first phase EF-G acts as a motor protein which transforms (part of) the energy of GTP hydrolysis into accelerated tRNA-mRNA movement on the ribosome, presumably by driving conformational changes of the ribosome. In the second phase EF-G switches to the GDP-bound conformation, which enables dissociation, and thus acts as a GTPase that couples the energy of GTP hydrolysis to operate conformational switching. 


\subsection{GTP hydrolysis and a functional domain 4 are required for synchronous translocation}

The present kinetic analysis of correlated tRNA movements during translocation on both $50 \mathrm{~S}$ and $30 \mathrm{~S}$ subunits provides new insight into translocation catalysis by EF-G (Figure 5-1). We show that binding of EF-G-GTP and GTP hydrolysis induces rapid concerted translocation of the tRNAs on both subunits. The rates of translocation of the tRNA and the mRNA coincide with the rate of Pi release (Savelsbergh et al., 2003). This is due to synchronization by the preceding rate-limiting step of $30 \mathrm{~S}$ unlocking (Savelsbergh et al., 2003), which presumably entails the opening of an mRNA-binding groove, allowing the mRNA and the anticodon domains of the tRNAs to pass through the $30 \mathrm{~S}$ subunit. This movement appears to be intrinsically rapid (Munro et al., 2010; Wen et al., 2008). EF-G might accelerate translocation by displacing ribosome elements that may act as hurdles for 30 S translocation (Schuwirth et al., 2005; Zhang et al., 2009) or by facilitating 30S head domain movements, the latter scenario being consistent with recent findings suggesting that the movement of the $30 \mathrm{~S}$ head coincides with mRNA movement (Guo and Noller, 2012, Ratje et al., 2010). Notably, the translocation rate can be increased by manipulating the decoding center of the $30 \mathrm{~S}$ subunit, e.g. by cleavage of $16 \mathrm{~S}$ rRNA between nucleotides A1493 and G1494 (Lancaster et al., 2008), by disrupting the interactions between the Asite codon and ribosomal residues in the decoding site (Khade and Joseph, 2011), or by altering the intersubunit bridges B1a, B4, B7a and B8 (Liu and Fredrick, 2013).

EF-G is a GTP-binding protein which combines the characteristics of a switch GTPase, which upon Pi release changes to a low-affinity conformation allowing the dissociation of the factor, with those of a motor that accelerates movement by a conformational change induced by GTP hydrolysis (described here and in Rodnina et al., 1997). The results obtained with EF-G(H91A) and EF-G(H583K) suggest that 50 S translocation proceeds in at least two steps $(\mathrm{A} / \mathrm{P} 1 \rightarrow \mathrm{A} / \mathrm{P} 2 \rightarrow \mathrm{P} / \mathrm{P} ;$ Figure $5-1)$. The first step, which is independent of $30 \mathrm{~S}$ translocation, moves the $3^{\prime}$ end of peptidyl-tRNA from the classic (C) or hybrid $(\mathrm{A} / \mathrm{P} 1)$ state into a state intermediate between pre-translocation and post-translocation; in that state, the reaction with puromycin remains slow, suggesting that the final post- 
translocation state is not reached yet. EF-G-GTP binding - independent of GTP hydrolysis - is sufficient to promote this movement, probably by affecting the landscape of spontaneous thermal motions within the ribosome. The energy of EF-G binding induces a global conformational change that controls concerted tRNA movements on both subunits through the dynamics of the $30 \mathrm{~S}$ head, but alone is not sufficient to promote rapid movement on the $30 \mathrm{~S}$ subunit. The second step, which entails $30 \mathrm{~S}$ translocation and tRNA movement on the $50 \mathrm{~S}$ subunit into the post-translocation state, is driven by GTP hydrolysis, which couples conformational rearrangements of EF-G to the engagement of domain 4 with the $30 \mathrm{~S}$ codon-anticodon complex. Domain 4 may promote $30 \mathrm{~S}$ translocation either directly, e.g. by altering the conformation of the ribosome, by opening the mRNA-binding cleft or by stabilizing the open conformation of the E-site gate (Borovinskaya et al., 2008, Ratje et al., 2010, Pulk and Cate, 2013, Tourigny et al., 2013). Alternatively, it may uncouple the mRNA-tRNA complex from two universally conserved bases in the ribosomal decoding center (Taylor et al., 2007) or restrict backward movement (Frank and Agrawal, 2000; Gao et al., 2009; Pulk and Cate, 2013; Tourigny et al., 2013; Zhou et al., 2013), thus coupling GTP hydrolysis to $30 \mathrm{~S}$ translocation in a way that resembles the power stroke of motor ATPases. This view is supported by the recent crystal structures of EF-G trapped on the ribosome in the pre-hydrolysis state (Pulk and Cate, 2013; Tourigny et al., 2013; Zhou et al., 2013) which predict that GTP hydrolysis should be associated with a rearrangement of EF-G that starts at the nucleotide binding pocket, pivots around domain 3 , and results in a movement of domain 4 and affects interactions with the $30 \mathrm{~S}$ subunit. One attractive possibility suggested by those structures is that GTP hydrolysis is required to allow for the backward rotation of the $30 \mathrm{~S}$ subunit head, which would complete the transition to the post-translocation state, and these is some evidence in favor of this suggestion from recent rapid kinetic experiments (as described and discussed above). Thus, the translocating ribosome-EF-G complex combines features of a Brownian machine and of a power-stroke motor. EF-G orchestrates the rapid synchronous progression of tRNAs and $\mathrm{mRNA}$ through the ribosome by combining the two energy regimes. 


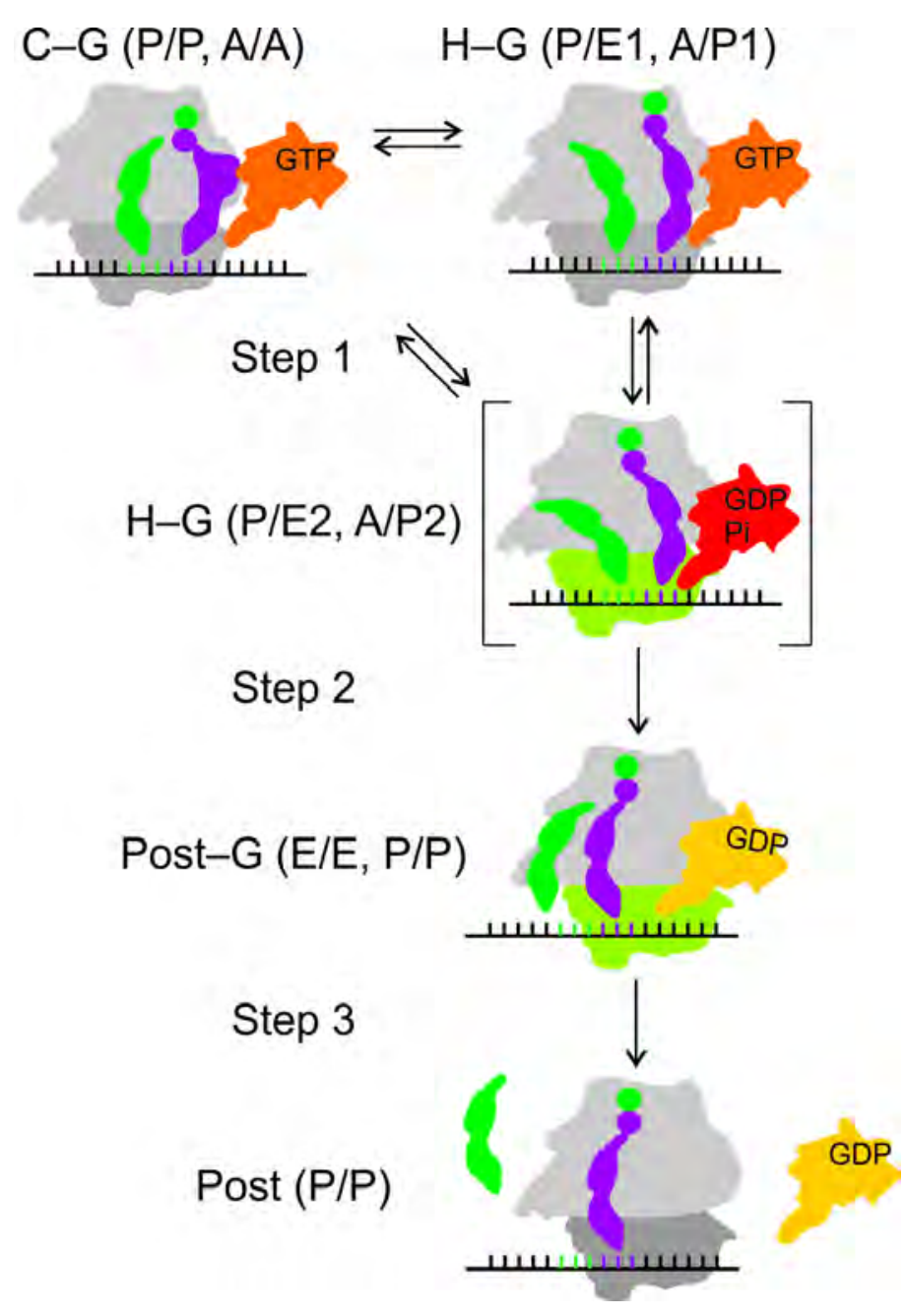

Figure 5-1: Schematic of translocation. EF-G-GTP bound to the pretranslocation complex in the classical $(\mathrm{C})$ state (with tRNAs in the $\mathrm{P} / \mathrm{P}$ and $\mathrm{A} / \mathrm{A}$ orientations) or in the hybrid/rotated state $(\mathrm{H})$ (with tRNA positions designated as $\mathrm{P} / \mathrm{E} 1$ and A/P1) (Chen et al., 2011, Walker et al., 2008), resulting in C-G or HG pre-translocation intermediates followed by rapid GTP hydrolysis. (Step 1) 30S unlocking (indicated by green 30S subunit) (Savelsbergh et al., 2003) concomitant with the movement of the P-site tRNA towards the E site (INT position (Pan et al., 2007); designated here as $\mathrm{P} / \mathrm{E} 2$ ) and the A-site tRNA into the intermediate position (A/P2). A/P2 state is observed with EF-G(H91A), and EF-G(583K) prior to $30 \mathrm{~S}$ translocation. The intermediate shown in square brackets normally is short-lived and formed also when there is no GTP hydrolysis. (Step 2) Translocation on the $30 \mathrm{~S}$ subunit and the completion of translocation on the 50S subunit, resulting in the post-translocation complex with tRNAs in the classical $\mathrm{E} / \mathrm{E}$ and $\mathrm{P} / \mathrm{P}$ states and EF-G occupying the A site (Post-G). (Step 3) Relocking of the 30S subunit and dissociation of EF-G and deacylated tRNA. Movements of individual elements of the ribosome (e.g. proteins L1, L11, and L12, or the 30S subunit head) and intermediate states of 30S translocation are not shown because they may be only loosely coupled to tRNA movement (Fischer et al., 2010) and their precise timing is uncertain. 


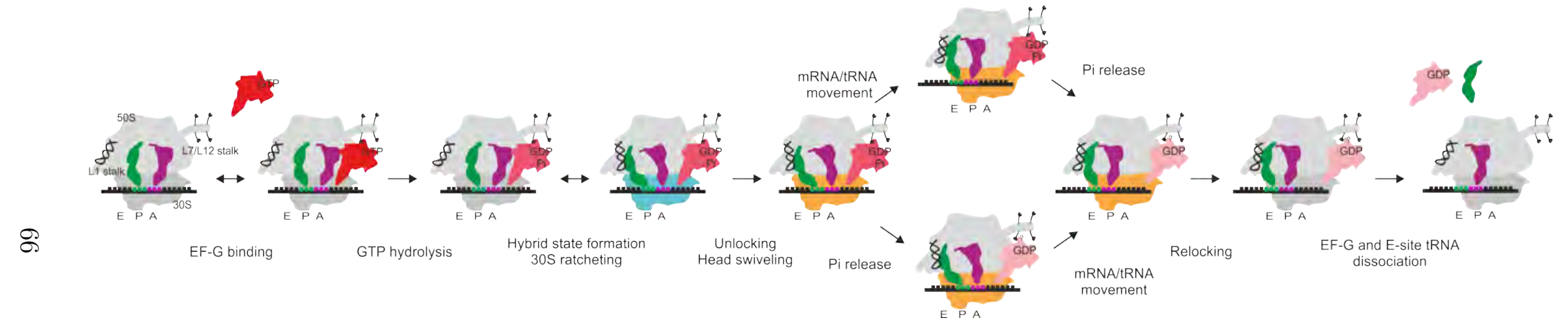

Figure 5-2: Translocation Model. EF-G is a probabilistic molecular motor that catalyzes unlocking and biases the system towards a post-translocated state. The model shows EF-G, the tRNAs and the ribosome undergo changes during translocation. EF-G-GTP, red; EF-G-GDP-Pi, dark pink; EF-G-GDP, light pink; deacylated tRNA, green; peptidyl tRNA, purple; 50S subunit, light grey; 30S subunit, dark grey; ratcheted 30S subunit, blue; unlocked 30S subunit, orange. 


\subsection{Conclusion}

In the light of all structural, biochemical and biophysical information discussed above and including the findings of the research papers presented here, a detailed model of translocation (Figure 5-2) is proposed. This model encompasses the role of the L1 stalk removing the deacylated tRNA from the $E$ site, as well as the role of $L 7 / L 12$ recruiting EF-G to the ribosome. The role of the $\mathrm{L} 7 / \mathrm{L} 12$ upon $\mathrm{Pi}$ release is not yet completely understood. EF-G undergoes different conformational changes during translocation.

At first, a conformational change resulting from GTP hydrolysis catalyzes unlocking, biasing the forward tRNA movement through conformational constraints. Upon Pi release, another conformational change in EF-G coupled with structural changes in the ribosome (relocking) results in factor dissociation.

The ribosome also undergoes many structural changes throughout the translocation cycle: (i) $30 \mathrm{~S}$ subunit ratcheting, which can take place spontaneously and is correlated to hybrid state formation, (ii) unlocking, which is the rate-limiting step of translocation and catalyzed by GTP hydrolysis, and (iii) 30S subunit head swiveling, which is loosely coupled to ratcheting and essential for mRNA/tRNA complex movement. All these movements are essential for translocation. In the absence of GTP hydrolysis, the ribosome/EF-G complex remains stalled in an intermediate conformation prior to Pi release. The subsequent relocking involves $30 \mathrm{~S}$ subunit head back-swiveling, $30 \mathrm{~S}$ subunit back-ratcheting and rearrangements leading to decreased affinity of EF-G to the ribosome. Finally, the model also shows that translocation occurs in a synchronous manner on the $50 \mathrm{~S}$ subunit and on the 30 subunit - as the final position of the tRNAs during the hybrid state is different from the one achieved after full translocation.

The energy regime of EF-G is also now better understood. It behaves a probabilistic molecular motor during translocation coupling the energy of GTP hydrolysis to a directional movement of the tRNAs, favoring the post-translocation state in detriment of the pre-translocation state. The delay between GTP hydrolysis and mRNA/tRNA complex translocation, the fact that translocation occurs spontaneously in the ribosome and that Pi can be released without an action motion provide support to this idea. Moreover, EF-G 
also behaves as a classical switch-type GTPase during its dissociation from ribosome EF-G is present on its 'on' conformation, being tightly bound to the ribosome, while the GDP-Pi state is kept; upon Pi release, EF-G-GDP is on its 'off' state, meaning that the affinity of EF-G to the ribosome decreases as well as this complex stability, leading to EF-G dissociation. This fact is supported by the high stability of EF-G(H91A) on the ribosome and other studies with non-hydrolyzable GTP analogs, vanadate mimicking Pi and when $\mathrm{Pi}$ remains bound to EF-G. Finally, it is not discarded that EF-G might still behave as a deterministic molecular machine during the unlocking step. It is known that GTP hydrolysis catalyzes unlocking, but how this is done is still unknown - one possibility is that upon a conformational change EF-G makes some contacts on the 30 S subunit, destabilizing the contacts in the intersubunit gap, leading to unlocking on a direct fashion. New structural data during the pre-translocation state might help to better understand this mechanism.

Nevertheless, despite the wealth of information from structural, biochemical and biophysical studies, there are still many open questions on the ribosome field: (i) how GTP hydrolysis is coupled to ribosome dynamics, (ii) how elongation factors are selected by the ribosome, and (iii) how GTPase activity of translation factors is achieved. Certainly, in the next years, with the development of finer single molecule microscopy techniques, more powerful processing tools and better sample preparations for cryo-EM, and new biophysical data from different labeling positions some of these questions will be addressed, allowing a deeper understanding of the global mechanistic functioning of the ribosome. 


\section{6}

\section{References}

AGIRREZABALA, X. \& FRANK, J. 2009. Elongation in translation as a dynamic interaction among the ribosome, tRNA, and elongation factors EF-G and EF-Tu. Q Rev Biophys, 42, 159-200.

AGRAWAL, R. K., PENCZEK, P., GRASSUCCI, R. A. \& FRANK, J. 1998. Visualization of elongation factor $\mathrm{G}$ on the Escherichia coli $70 \mathrm{~S}$ ribosome: the mechanism of translocation. Proc Natl Acad Sci U S A, 95, 6134-8.

AGTHOVEN, A. J., MAASSEN, J. A., SCHRIER, P. I. \& MOLLER, W. 1975. Inhibition of EF-G dependent GTPase by an aminoterminal fragment of L7/L12. Biochem Biophys Res Commun, 64, 1184-91.

BARCISZEWSKA, M. Z., SZYMANSKI, M., ERDMANN, V. A. \& BARCISZEWSKI, J. 2001. Structure and functions of 5S rRNA. Acta Biochim Pol, 48, 191-8.

BERGEMANN, K. \& NIERHAUS, K. H. 1983. Spontaneous, elongation factor G independent translocation of Escherichia coli ribosomes. J Biol Chem, 258, 15105-13.

BERK, V. \& CATE, J. H. 2007. Insights into protein biosynthesis from structures of bacterial ribosomes. Curr Opin Struct Biol, 17, 302-9.

BLANCHARD, S. C., KIM, H. D., GONZALEZ, R. L., JR., PUGLISI, J. D. \& CHU, S. 2004. tRNA dynamics on the ribosome during translation. Proc Natl Acad Sci U S A, 101, 12893-8. 
BOROVINSKAYA, M. A., SHOJI, S., HOLTON, J. M., FREDRICK, K. \& CATE, J. H. 2007. A steric block in translation caused by the antibiotic spectinomycin. ACS Chem Biol, 2, $545-52$.

BOURNE, H. R., SANDERS, D. A. \& MCCORMICK, F. 1990. The GTPase superfamily: a conserved switch for diverse cell functions. Nature, 348, 125-32.

BOURNE, H. R., SANDERS, D. A. \& MCCORMICK, F. 1991. The GTPase superfamily: conserved structure and molecular mechanism. Nature, 349, 117-27.

BROT, N., TATE, W. P., CASKEY, C. T. \& WEISSBACH, H. 1974. The requirement for ribosomal proteins L7 and L12 in peptide-chain termination. Proc Natl Acad Sci U S A, $71,89-92$.

BRUNE, M., HUNTER, J. L., CORRIE, J. E. \& WEBB, M. R. 1994. Direct, real-time measurement of rapid inorganic phosphate release using a novel fluorescent probe and its application to actomyosin subfragment 1 ATPase. Biochemistry, 33, 8262-71.

BUBUNENKO, M. G., CHUIKOV, S. V. \& GUDKOV, A. T. 1992. The length of the interdomain region of the L7/L12 protein is important for its function. FEBS Lett, 313, 232-4.

CALDON, C. E. \& MARCH, P. E. 2003. Function of the universally conserved bacterial GTPases. Curr Opin Microbiol, 6, 135-9.

CARTER, A. P., Clemons, W. M., BRODERSEN, D. E., MORGAN-WARREN, R. J., WIMBERLY, B. T. \& RAMAKRISHNAN, V. 2000. Functional insights from the structure of the $30 \mathrm{~S}$ ribosomal subunit and its interactions with antibiotics. Nature, 407, 340-348.

ChEN, J., PETROV, A., TSAI, A., O'LeARY, S. E. \& PUGLISI, J. D. 2013. Coordinated conformational and compositional dynamics drive ribosome translocation. Nat Struct Mol Biol.

CONNELL, S. R., TAKEMOTO, C., WILSON, D. N., WANG, H., MURAYAMA, K., TERADA, T., SHIROUZU, M., ROST, M., SCHULER, M., GIESEBRECHT, J., DABROWSKI, M., MIELKE, T., FUCINI, P., YOKOYAMA, S. \& SPAHN, C. M. 2007. Structural basis for 
interaction of the ribosome with the switch regions of GTP-bound elongation factors. Mol Cell, 25, 751-64.

CUNHA, C. E., BELARDINELLI, R., PESKE, F., HOLTKAMP, W., WINTERMEYER, W. \& RODNINA, M. V. 2013. Dual use of GTP hydrolysis by elongation factor $G$ on the ribosome. Translation, 1, e24315.

CUKRAS, A. R., SOUThWORTH, D. R., BRUNELlE, J. L., CULVER, G. M. \& GREEN, R. 2003. Ribosomal proteins S12 and S13 function as control elements for translocation of the mRNA:tRNA complex. Mol Cell, 12, 321-8.

CZWORKOWSKI, J. \& MOORE, P. B. 1997. The conformational properties of elongation factor $\mathrm{G}$ and the mechanism of translocation. Biochemistry, 36, 10327-34.

DAVITER, T., WIEDEN, H. J. \& RODNINA, M. V. 2003. Essential role of histidine 84 in elongation factor Tu for the chemical step of GTP hydrolysis on the ribosome. J Mol Biol, 332, 689-99.

DAVYDOV, II, WOHLGEMUTH, I., ARTAMONOVA, II, URLAUB, H., TONEVITSKY, A. G. \& RODNINA, M. V. 2013. Evolution of the protein stoichiometry in the L12 stalk of bacterial and organellar ribosomes. Nat Commun, 4, 1387.

DIACONU, M., KOTHE, U., SCHLUNZEN, F., FISCHER, N., HARMS, J. M., TONEVITSKY, A. G., STARK, H., RODNINA, M. V. \& WAHL, M. C. 2005. Structural basis for the function of the ribosomal L7/12 stalk in factor binding and GTPase activation. Cell, 121 , 991-1004.

DOERFEL, L. K., WOHLGEMUTH, I., KOTHE, C., PESKE, F., URLAUB, H. \& RODNINA, M. V. 2013. EF-P is essential for rapid synthesis of proteins containing consecutive proline residues. Science, 339, 85-8.

DORNER, S., BRUNELLE, J. L., SHARMA, D. \& GREEN, R. 2006. The hybrid state of tRNA binding is an authentic translation elongation intermediate. Nat Struct Mol Biol, $13,234-41$. 
ELLIS, J. P., BAKKE, C. K., KIRCHDOERFER, R. N. JUNGBAUER, L. M. \& CAVAGBERO, S. 2008. Chain dynamics of nascent polypeptides emerging from the ribosome. ACS Chem Biol, 3, 555-566.

ERMOLENKO, D. N., MAJUMDAR, Z. K., HICKERSON, R. P., SPIEGEL, P. C., CLEGG, R. M. \& NOLLER, H. F. 2007. Observation of intersubunit movement of the ribosome in solution using FRET. J Mol Biol, 370, 530-40.

ERMOLENKO, D. N. \& NOLLER, H. F. 2011. mRNA translocation occurs during the second step of ribosomal intersubunit rotation. Nat Struct Mol Biol, 18, 457-462.

EVANS, R. N., BLAHA, G., BAILEY, S. \& STEITZ, T. A. 2008. The structure of LepA, the ribosomal back translocase. Proc Natl Acad Sci U S A, 105, 4673-8.

FAKUNDING, J. L., TRAUT, R. R. \& HERSHEY, J. W. 1973. Dependence of initiation factor IF-2 activity on proteins L7 and L12 from Escherichia coli $50 \mathrm{~S}$ ribosomes. J Biol Chem, 248, 8555-9.

FEI, J., KOSURI, P., MACDOUGALL, D. D. \& GONZALEZ, R. L., JR. 2008. Coupling of ribosomal L1 stalk and tRNA dynamics during translation elongation. Mol Cell, 30, 348-59.

FISCHER, N., KONEVEGA, A. L., WINTERMEYER, W., RODNINA, M. V. \& STARK, H. 2010. Ribosome dynamics and tRNA movement by time-resolved electron cryomicroscopy. Nature, 466, 329-33.

FRANK, J. \& AGRAWAL, R. K. 2000. A ratchet-like inter-subunit reorganization of the ribosome during translocation. Nature, 406, 318-22.

FRANK, J., GAO, H., SENGUPTA, J., GAO, N. \& TAYLOR, D. J. 2007. The process of mRNA-tRNA translocation. Proc Natl Acad Sci U S A, 104, 19671-8.

GAO, Y. G., SElMER, M., DUnhAM, C. M., WeiXlBAumer, A., KELleY, A. C. \& RAMAKRISHNAN, V. 2009. The structure of the ribosome with elongation factor $G$ trapped in the posttranslocational state. Science, 326, 694-9.

GITE, S., MAMAEV, S., OLEJNIK, J. \& ROTHSCHILD, K. 2000. Ultrasensitive fluorescencebased detection of nascent proteins in gels. Anal Biochem, 279, 218-225. 
GORLICH, D. \& KUTAY, U. 1999. Transport between the cell nucleus and the cytoplasm. Annu Rev Cell Dev Biol, 15, 607-60.

GUDKOV, A. T. 1997. The L7/L12 ribosomal domain of the ribosome: structural and functional studies. FEBS Lett, 407, 253-6.

GUDKOV, A. T. \& BUBUNENKO, M. G. 1989. Conformational changes in ribosomes upon interaction with elongation factors. Biochimie, 71, 779-85.

GUDKOV, A. T., BUBUNENKO, M. G. \& GRYAZNOVA, O. I. 1991. Overexpression of L7/L12 protein with mutations in its flexible region. Biochimie, 73, 1387-9.

GUDKOV, A. T. \& GONGADZE, G. M. 1984. The L7/L12 proteins change their conformation upon interaction of EF-G with ribosomes. FEBS Lett, 176, 32-6.

GUO, Z. \& NOLLER, H. F. 2012. Rotation of the head of the 30 S ribosomal subunit during mRNA translocation. Proc Natl Acad Sci USA, 109,20391-4.

HAMEL, E., KOKA, M. \& NAKAMOTO, T. 1972. Requirement of an Escherichia coli 50 $S$ ribosomal protein component for effective interaction of the ribosome with $T$ and $G$ factors and with guanosine triphosphate. J Biol Chem, 247, 805-14.

hOlTKAMP, W., CUNHA, C. E., PESKE, F., KONEVEGA, A. L., WINTERMEYER, W. \& RODNINA, M. V. 2013. GTP hydrolysis by Elongation Factor G Synchronizes Translocation on the Two Ribosomal Subunits. Manuscript submitted and under review

HICKERSON, R., MAJUMBAR, Z. K., BAUCOM, A., CleGG, R. M. \& NOlLeR. H. F. 2005. Measurement of internal movements within the $30 \mathrm{~S}$ ribosomal subunit using Frster resonance energy transfer. J Mol Biol, 354, 459-72.

HIRASHIMA, A. \& KAJI, A. 1973. Role of elongation factor $G$ and a protein factor on the release of ribosomes from messenger ribonucleic acid. J Biol Chem, 248, 7580-7.

HORAN, L. H. \& NOLLER, H. F. 2007. Intersubunit movement is required for ribosomal translocation. Proc Natl Acad Sci U S A, 104, 4881-5.

HUXLEY, A. F. 2000. Mechanics and models of the myosin motor. Philos Trans R Soc Lond B Biol Sci, 355, 433-40. 
JORGENSEN, R., ORTIZ, P. A., CARR-SCHMID, A., NISSEN, P., KINZY, T. G. \& ANDERSEN, G. R. 2003. Two crystal structures demonstrate large conformational changes in the eukaryotic ribosomal translocase. Nat Struct Biol, 10, 379-85.

KATUNIN, V. I., SAVELSBERGH, A., RODNINA, M. V. \& WINTERMEYER, W. 2002. Coupling of GTP hydrolysis by elongation factor $G$ to translocation and factor recycling on the ribosome. Biochemistry, 41, 12806-12.

KAY, A., SANDER, G. \& GRUNBERG-MANAGO, M. 1973. Effect of ribosomal protein L12 upon initiation factor IF-2 activities. Biochem Biophys Res Commun, 51, 979-86.

KELLER, D. \& BUSTAMANTE, C. 2000. The mechanochemistry of molecular motors. Biophys J, 78, 541-56.

KIM, H. D., PUGLISI, J. D. \& CHU, S. 2007. Fluctuations of transfer RNAs between classical and hybrid states. Biophys J, 93, 3575-82.

KISCHA, K., MOLLER, W. \& STOFFLER, G. 1971. Reconstitution of a GTPase activity by a 50 S ribosomal protein and E. coli. Nat New Biol, 233, 62-3.

KONEVEGA, A. L., FISCHER, N., SEMENKOV, Y. P., STARK, H., WINTERMEYER, W. \& RODNINA, M. V. 2007. Spontaneous reverse movement of mRNA-bound tRNA through the ribosome. Nat Struct Mol Biol, 14, 318-324.

KOPKE, A. K., LEGGATT, P. A. \& MATHESON, A. T. 1992. Structure function relationships in the ribosomal stalk proteins of archaebacteria. J Biol Chem, 267, 1382-90.

KOTHE, U., WIEDEN, H. J., MOHR, D. \& RODNINA, M. V. 2004. Interaction of helix D of elongation factor Tu with helices 4 and 5 of protein $\mathrm{L} 7 / 12$ on the ribosome. J Mol Biol, 336, 1011-21.

LANCASTER, L.E., SAVELSBERGH, A., KLEANTHOUS, C., WINTERMEYER, W., \& RODNINA, M.V. 2008. Colicin E3 cleavage of 16S rRNA impairs decoding and accelerates tRNA translocation on Escherichia coli ribosomes. Mol Microbiol 69, 390-401.

LEIJONMARCK, M. \& LILJAS, A. 1987. Structure of the C-terminal domain of the ribosomal protein L7/L12 from Escherichia coli at 1.7 A. J Mol Biol, 195, 555-79. 
LILJAS, A. \& GUDKOV, A. T. 1987. The structure and dynamics of ribosomal protein L12. Biochimie, 69, 1043-7.

LIU, Q. \& FREDRICK, K. 2013. Contribution of intersubunit bridges to the energy barrier of ribosomal translocation. Nucl Acids Res 41, 565-574.

MILON, P., KONEVEGA, A. K., PeSKe, F., FABBReTtI, A., GUAlerZI, C. O. \& RODNINA, M. V. 2007. Transient kinetics, fluorescence, and FRET in studies of initiation of translation in bacteria. Methods Enzymol, 430, 1-30

MILON, P. \& RODNINA, M. V. 2012. Kinetic control of translation initiation in bacteria. Crit Rev Biochem Mol Biol, 47, 334-48.

MOAZED, D. \& NOLLER, H. F. 1989. Intermediate states in the movement of transfer RNA in the ribosome. Nature, 342, 142-8.

MOHR, D., WINTERMEYER, W. \& RODNINA, M. V. 2002. GTPase activation of elongation factors Tu and G on the ribosome. Biochemistry, 41, 12520-8.

MUNRO, J. B., ALTMAN, R. B., O'CONNOR, N. \& BLANCHARD, S. C. 2007. Identification of two distinct hybrid state intermediates on the ribosome. Mol Cell, 25, 505-17.

MUNRO, J. B., WASSERMAN, M. R., ALTMAN, R. B., WANG, L. \& BLANCHARD, S. C. 2010. Correlated conformational events in EF-G and the ribosome regulate translocation. Nat Struct Mol Biol, 17, 1470-1477.

NISSEN, P., KJELDGAARD, M. \& NYBORG, J. 2000. Macromolecular mimicry. EMBO J, 19, 489-95.

OleinikOV, A. V., PERROUD, B., WANG, B. \& TRAUT, R. R. 1993. Structural and functional domains of Escherichia coli ribosomal protein L7/L12. The hinge region is required for activity. J Biol Chem, 268, 917-22.

PAN, D., KIRILLOV, S. V. \& COOPERMAN, B. S. 2007. Kinetically competent intermediates in the translocation step of protein synthesis. Mol Cell, 25, 519-29. 
PESKE, F., MATASSOVA, N. B., SAVELSBERGH, A., RODNINA, M. V. \& WINTERMEYER, W. 2000. Conformationally restricted elongation factor G retains GTPase activity but is inactive in translocation on the ribosome. Mol Cell, 6, 501-5.

PESKE, F., SAVELSBERGH, A., KATUNIN, V. I., RODNINA, M. V. \& WINTERMEYER, W. 2004. Conformational changes of the small ribosomal subunit during elongation factor G-dependent tRNA-mRNA translocation. J Mol Biol, 343, 1183-94.

PULK, A. \& CATE, J.H. 2013. Control of ribosomal subunit rotation by elongation factor G. Science $x x x, x x x-x x x$.

RATJE, A. H., LOERKE, J., MIKOLAJKA, A., BRUNNER, M., HILDEBRAND, P. W., Starosta, A. L., DONhOFER, A., CONNELl, S. R., FUCINI, P., MIELKE, T., WHITFORD, P. C., ONUCHIC, J. N., YU, Y., SANBONMATSU, K. Y., HARTMANN, R. K., PENCZEK, P. A., WILSON, D. N. \& SPAHN, C. M. 2010. Head swivel on the ribosome facilitates translocation by means of intra-subunit tRNA hybrid sites. Nature, 468, 713-6.

RODNINA, M. V., BERINGER, M. \& WINTERMEYER, W. 2007. How ribosomes make peptide bonds. Trends Biochem Sci, 32, 20-6.

RODNINA, M. V., PAPE, T., FRICKE, R., KUHN, L. \& WINTERMEYER, W. 1996. Initial binding of the elongation factor Tu.GTP.aminoacyl-tRNA complex preceding codon recognition on the ribosome. J Biol Chem, 271, 646-52.

RODNINA, M. V., SAVELSBERGH, A., KATUNIN, V. I. \& WINTERMEYER, W. 1997. Hydrolysis of GTP by elongation factor G drives tRNA movement on the ribosome. Nature, $385,37-41$.

RODNINA, M. V. \& WINTERMEYER, W. 2001. Ribosome fidelity: tRNA discrimination, proofreading and induced fit. Trends Biochem Sci, 26, 124-30.

RODNINA, M. V. \& WINTERMEYER, W. 1995. GTP consumption of elongation factor Tu during translations of heteropolymeric mRNAs. Proc Natl Acad Sci USA, 92, 1945-9.

RODNINA, M. V. \& WINTERMEYER, W. 2011. The ribosome as a molecular machine: the mechanism of tRNA-mRNA movement in translocation. Biochem Soc Trans, 39, 658-62. 
SAVELSBERGH, A., KATUNIN, V. I., MOHR, D., PESKE, F., RODNINA, M. V. \& WINTERMEYER, W. 2003. An elongation factor G-induced ribosome rearrangement precedes tRNA-mRNA translocation. Mol Cell, 11, 1517-23.

SAVELSBERGH, A., MATASSOVA, N. B., RODNINA, M. V. \& WINTERMEYER, W. 2000a. Role of domains 4 and 5 in elongation factor $G$ functions on the ribosome. J Mol Biol, $300,951-61$.

SAVELSBERGH, A., MOHR, D., KOTHE, U., WINTERMEYER, W. \& RODNINA, M. V. 2005. Control of phosphate release from elongation factor $G$ by ribosomal protein $L 7 / 12$. EMBO J, 24, 4316-23.

SAVELSBERGH, A., MOHR, D., WILDEN, B., WINTERMEYER, W. \& RODNINA, M. V. 2000b. Stimulation of the GTPase activity of translation elongation factor G by ribosomal protein L7/12. J Biol Chem, 275, 890-4.

SAVELSBERGH, A., RODNINA, M. V. \& WINTERMEYER, W. 2009. Distinct functions of elongation factor $\mathrm{G}$ in ribosome recycling and translocation. RNA, 15, 772-80.

SCHMEING, T. M. \& RAMAKRISHNAN, V. 2009. What recent ribosome structures have revealed about the mechanism of translation. Nature, 461, 1234-42.

SEMENKOV, Y. P., RODNINA, M. V. \& WINTERMEYER, W. 2000. Energetic contribution of tRNA hybrid state formation to translocation catalysis on the ribosome. Nat Struct Biol, 7, 1027-31.

SCHUWIRTH, B. S., BOROVINSKAYA, M. A., HAU, C. W., ZHANG, W., VILA-SANJURJO, A. HOLTON, J. M. \& CATE, J. H. 2005. Structures of the bacterial ribosome at 3.5Åresolution. Science 310, 827-834.

SEO, H.S., ABEDIN, S., KAMP, D., WILSON, D. N., NIERHAUS, K. H. \& COOPERMAN, B. S. 2006. EF-G-dependent GTPase on the ribosome - conformational change and fusidic acid inhibition. Biochemistry, 45, 2504-14.

SERGIEV, P. V., BOGDANOV, A. A. \& DONTSOVA, O. A. 2005. How can elongation factors EF-G and EF-Tu discriminate the functional state of the ribosome using the same binding site? FEBS Lett, 579, 5439-42. 
SHARMA, D., SOUTHWORTH, D. R. \& GREEN, R. 2004. EF-G-independent reactivity of a pre-translocation-state ribosome complex with the aminoacyl tRNA substrate puromycin supports an intermediate (hybrid) state of tRNA binding. RNA, 10, 102-13.

SHI, X., KHADE, P. K., SANBONMATSU, K. Y. \& JOSEPH, S. 2012. Functional role of the sarcin-ricin loop of the $23 \mathrm{~S}$ rRNA in the elongation cycle of protein synthesis. J Mol Biol, $419,125-38$.

SHOJI, S., WALKER, S. E. \& FREDRICK, K. 2009. Ribosomal translocation: one step closer to the molecular mechanism. ACS Chem Biol, 4, 93-107.

SOMMER, A., ETCHISON, J. R., GAVINO, G., ZECHERLE, N., CASIANO, C. \& TRAUT, R. R. 1985. Preparation and characterization of two monoclonal antibodies against different epitopes in Escherichia coli ribosomal protein L7/L12. J Biol Chem, 260, 6522-7.

SPAHN, C. M., GOMEZ-LORENZO, M. G., GRASSUCCI, R. A., JORGENSEN, R., ANDERSEN, G. R., BECKMANN, R., PENCZEK, P. A., BALLESTA, J. P. \& FRANK, J. 2004. Domain movements of elongation factor eEF2 and the eukaryotic 805 ribosome facilitate tRNA translocation. EMBO J, 23, 1008-19.

SPIEGEL, P. C., ERMOLENKO, D. N. \&NOLLER, H. F. 2007. Elongation factor G stabilizes the hybrid-state conformation of the 70 S ribosome. RNA, 13, 1473-82

STARK, H., RODNINA, M. V., WIEDEN, H. J., VAN HEEL, M. \& WINTERMEYER, W. 2000. Large-scale movement of elongation factor $G$ and extensive conformational change of the ribosome during translocation. Cell, 100, 301-9.

STUDER, S. M., FEINBERG, J. S., \& JOSEPH, S. 2003. Rapid kinetic analysis of EF-Gdependent mRNA translocation in the ribosome. J Mol Biol, 327, 369-381.

TAKYAR, S., HICKERSON, R. P. \& NOLLER, H. F. 2005. mRNA helicase activity of the ribosome. Cell, 120, 49-58.

TAYLOR, D.J., NILSSON, J., MERRILL, A.R., ANDERSEN, G.R., NISSEN, P. \& FRANK, J. 2007. Structures of modified eEF2-80S ribosome complexes reveal the role of GTP hydrolysis in translocation. Embo J 26, 2421-2431. 
TOURIGNY, D.S., FERNNDEZ, I.S., KELLEY, A.C. \& RAMAKRISHNAN, V. 2013. Elongation factor $G$ bound to the ribosome in am intermediate state of translocation. Science $x x x$.

TRABUCO, L. G., SCHREINER, E., EARGLE, J., CORNISH, P., HA, T., LUTHEY-SCHULTEN, Z. \& SCHULTEN, K. 2010. The role of L1 stalk-tRNA interaction in the ribosome elongation cycle. J Mol Biol, 402, 741-60.

UDE, S., LASSAK, J., STAROSTA, A. L., KRAXENBERGER, T., WILSON, D. N. \& JUNG, K. 2013. Translation elongation factor EF-P alleviates ribosome stalling at polyproline stretches. Science, 339, 82-5.

VAlle, M., ZAVIALOV, A., SENGUPTA, J., RAWAT, U., EHRENBERG, M. \& FRANK, J. 2003. Locking and unlocking of ribosomal motions. Cell, 114, 123-34.

VERSTRAETEN, N., FAUVART, M., VERSEES, W. \& MICHIELS, J. 2011. The universally conserved prokaryotic GTPases. Microbiol Mol Biol Rev, 75, 507-42, second and third pages of table of contents.

VOGEley, L., PALM, G. J., MESTERS, J. R. \& HILGENFELD, R. 2001. Conformational change of elongation factor Tu (EF-Tu) induced by antibiotic binding. Crystal structure of the complex between EF-Tu-GDP and aurodox. J Biol Chem, 276, 17149-55.

VOORHEES, R. M., SCHMEING, T. M., KELLEY, A. C. \& RAMAKRISHNAN, V. 2010. The mechanism for activation of GTP hydrolysis on the ribosome. Science, 330, 835-8.

WAHL, M. C., BOURENKOV, G. P., BARTUNIK, H. D. \& HUBER, R. 2000. Flexibility, conformational diversity and two dimerization modes in complexes of ribosomal protein L12. EMBO J, 19, 174-86.

WAHL, M. C. \& MOLLER, W. 2002. Structure and function of the acidic ribosomal stalk proteins. Curr Protein Pept Sci, 3, 93-106.

WALKER, S. E., SHOJI, S., PAN, D., COOPERMAN, B. S. \& FREDRICK, K. 2008. Role of hybrid tRNA-binding states in ribosomal translocation. Proc Natl Acad Sci U S A, 105, 9192-7. 
WALLIN, G., KAMERLIN, S. C. \& AQVIST, J. 2013. Energetics of activation of GTP hydrolysis on the ribosome. Nat Commun, 4, 1733.

WEN, J. D., LANCASTER, L., HODGES, C., ZERI, A. C., YOSHIMURA, S. H., NOLLER, H. F., BUSTAMANTE, C., \& TINOCO, I. 2008. Following translation by single ribosomes one codon at a time. Nature 452, 598-603.

WILDEN, B., SAVELSBERGH, A., RODNINA, M. V. \& WINTERMEYER, W. 2006. Role and timing of GTP binding and hydrolysis during EF-G-dependent tRNA translocation on the ribosome. Proc Natl Acad Sci USA, 103, 13670-5

WILSON, K. S. \& NOLLER, H. F. 1998. Mapping the position of translational elongation factor EF-G in the ribosome by directed hydroxyl radical probing. Cell, 92, 131-9.

WINTERMEYER, W., PESKE, F., BERINGER, M., GROMADSKI, K. B., SAVELSBERGH, A. \& RODNINA, M. V. 2004. Mechanisms of elongation on the ribosome: dynamics of a macromolecular machine. Biochem Soc Trans, 32, 733-7.

WINTERMEYER, W. \& RODNINA, M. V. 2000. Translational elongation factor G: a GTPdriven motor of the ribosome. Essays Biochem, 35, 117-29.

WINTERMEYER, W., SCHLEICH, H. G. \& ZACHAU, H. G. 1979a. Incorporation of amines or hydrazines into tRNA replacing wybutine or dihydrouracil. Methods Enzymol, 59, 110-21.

WINTERMEYER, W. \& ZACHAU, H. G. 1979b. Fluorescent derivatives of yeast tRNA ${ }^{\text {Phe }}$. Eur J Biochem, 98, 465-75.

ZAVIALOV, A. V. \& EHRENBERG, M. 2003. Peptidyl-tRNA regulates the GTPase activity of translation factors. Cell, 114, 113-22.

ZHANG, W., DUNKLE, J. A. \& CATE, J. H. 2009. Structures of the ribosome in intermediate states of ratcheting. Science 325, 1014-1017.

ZHOU, J., LANCASTER, L., DONOHUE, J.P. \& NOLLER, H.F. 2013. Crystal structures of EF-G-ribosome complexes trapped in intermediate states of translocation. Science xxx, $x x x-x x x$. 
ZHOU, J., LANCASTER, L., TRAKHANOV, S. \& NOLLER, H. F. 2012. Crystal structure of release factor RF3 trapped in the GTP state on a rotated conformation of the ribosome. RNA, 18, 230-40. 
7

\section{Biography}




\section{Carlos Eduardo Lima da Cunha}

\section{Biography}

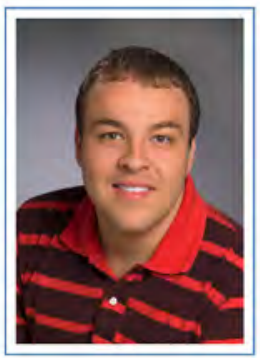

\section{Born in São Paulo, Brazil Education}

on August, $16^{\text {th }} 1984$

Ph.D. Student, International Max-Planck Research

2008-2013

School for Molecular Biology - Georg-August Universität

Göttingen, Göttingen, Germany.

Bachelor of Science, Federal University of São Paulo,

São Paulo, Brazil, Specialized in Physics, Biophysics and

Bioinformatics.

A-Level, American School and College MackenzieTamboré and Franciscan School Pio XII, São Paulo, Brazil.

\section{Experience}

Publications

Kinetic characterization of the Escherichia coli oligopeptidase $A(O p d A)$ and the role of the $\operatorname{Tyr}(607)$ residue, Lorenzon, R.Z., Cunha, C.E., Marcondes, M.F., Machado, M.F., Juliano, M.A., Oliveira, V., Travassos, L.R., Paschoalin \& T., Carmona, A.K., Arch. Biochem. Biophys.

How to get the magic triangle and the MAD triangle into your protein crystal, BECK, T., CUNHA, C.E. \& SHELDRICK, G.M., Acta Crystallogr. Sect. F Struct. Biol. Cryst. Commun.

Catalytic properties of recombinant dipeptidyl carboxypeptidase from Escherichia coli: a comparative study with angiotensin I-converting enzyme, CUNHA, C.E., Magliarelli, H.F., Paschoalin, T., Nchinda, A.T., Lima, J.C., Juliano, M.A., Paiva, P.B., Sturrock, E.D., Travassos, L.R. \& Carmona, A.K., Biol. Chem.

\section{Awards}

2007: CNPq Award - national award for best undergrad-conducted research

2006: Pereira Barreto Award - university award for best undergradconducted research 